Seismic Risk in the Assam Gap, Eastern Himalaya

\author{
K. Khattri \\ Max Wyss \\ University of Colorado \\ CIRES \\ Boulder, Colorado 80309 \\ USGS CONTRACT NO. 14-08-0001-16841
}

Supported by the EARTHQUAKE HAZARDS REDUCTION PROGRAM

OPEN-FILE NO. 80-2011

U.S. Geological Survey

OPEN FILE REPORT

This report was prepared under contract to the U.S. Geological Survey and has not been reviewed for conformity with USGS editorial standards and stratigraphic nomenclature. Opinions and conclusions expressed herein do not necessarily represent those of the USGS. Any use of trade names is for descriptive purposes only and does not imply endorsement by the USGS: 
Contract Number: 14-08-0001-16841

Name of Contractor: University of Colorado

Principal Investigator: Max Wyss and $\mathrm{K}$. Khattri

Government Technical officer: Jack E. Evernden

Title of work: Seismic Risk in the Assam Gap, Eastern Himalaya

Effective date of Contract: 1 May 1978

Contract Expiration Date: 31 February 1980

Amount of Contract: $\$ 31,520$

FINAL TECHNICAL REPORT

Sponsored by the

U.S. Geological survey

Contract No. 14-08-0001-16841 
SUMMARY

SEISMIC RISK IN THE ASSAM GAP

K.N. KHATTRI ${ }^{\text {I) }}$ and M. WYSS ${ }^{2)}$

The Himalaya form a clearly defined arcuate zone of plate consumption along which the Indian and Asian Plates collide at a rate of about $5 \mathrm{~cm} /$ year. In the eastern part of this plate boundary a $500 \mathrm{~km}$ segment, lying between the epicenters of the great 1897 and 1950 earthquakes, is considered to be a seismic gap. A three station seismograph network with a diameter of about $100 \mathrm{~km}$ was operated in the western part of the Assam gap for 5 months during 1979. The main purpose of this pilot program was to determine whether high quality seismograms for earthquake prediction research could be obtained in this area.

It was found that the general area is seismically fairly active. On the average, 20 local earthquakes per day were recorded. Their coda duration times varied between 5 and 200 seconds, with most events having a signal duration length of around 80 seconds. Approximately $25 \%$ of these events could be located within the three-station network. The seismograph data from Shillong turned out to be of limited use, because few of these events are recorded there, due to the relatively low frequency ( $1 \mathrm{cps}$ ) response of the WWSSN seismographs.

Out of 425 station-days recording time 87 station-days were lost due to various reasons. This shows that seismic networks can be operated in the Assam area with a down-time of about $20 \%$. With a ten station network, one would thus expect 8 stations to run coincidentally. To date, 124 earthquakes have been located. Several hundred events are in the process of being analysed.

1) Earth Sciences Department, Roorkee University, Roorkee U.P., India.

2) Cooperative Inst. Research Environmental Science, Univ. of Colorado, Boulder, Colorado 80309, U.S.A. 
At present a six station network is being installed in the Assam area under a contract funded by the U.S. Geological Survey to continue the work reported here. Several other Indian institutions carry out geophysical research in the area as an integral part of a national effort to evaluate the seismic risk in the region. It is concluded that the U.S.G.S. funded study of the seismicity and physics of the earthquake source properties and other factors, which would make a fundamental contribution to the assessment of the earthquake risk in the Assam gap, can be carried out successfully and that this will consitute an important part of the larger overall project. 
A. Functional subjects $\quad \cdot 4$

B. Discussion 4

1. Premobilization Phase I 4

2. Premobilization Phase II 4

3. Mobilization Phase I 4

4. Mobilization Phase II: Field Operation 4

4a. Introduction 4

4b. Field operations 6

4c. Research Programs conducted by other agencies 9

5. Data Analysis 11

6. Conclusions and Recommendations 15

Appendix A: Examples of Seismograms

Appendix B: List of arrival times

Appendix C: List of preliminary hypocenters

Appendix D: Article published in Geology 
1. Premobilization Phase I: Study of the historic seismicity in northeastern India.

2. Premobilization Phase II: Purchase and testing of seismographs.

3. Mobilization Phase I: Detailed inspection of field conditions, arrangements for logistic support, processing of permit for fieldwork in the study area.

4. Mobilization Phase II: Installation of seismographs in the field and continuous operation of the network.

5. Data Analysis.

B.

\section{DISCUSSION}

1. Premobilization Phase I

The study of the historic seismicity in the Assam area has been completed. A publication on that subject by the principal investigators appeared in the November issue of Geology (Appendix L).

\section{Premobilization Phase II}

The purchase and field testing of the seismographs has been completed. Funds from other sources were acquired to ship the instruments to Iceland for the testing.

\section{Mobilization Phase I}

Funds were obtained from the Smithsonian Institution for travel to India by $M$. Wyss in order to examine local environmental conditions and logistic support needed for the project as well as to determine when approval for the project by the Indian government could be expected.

4. Mobilization Phase II: Field operations.

\section{(4a.) Introduction}

The Assam seismic gap extending for over $500 \mathrm{~km}$. in a roughly NE-WSW direction lies between the epicenters of the two great Assam Earthquakes: that of 1897 in the west and that of 1950 which occurred at the North Eastern 
syntaxial bend of the Himalayan Arc. The 1897 earthquake occurred in an area known as the Meghalaya (formerly Shillong) plateau which is formed of ancient complex Archean metamorphics. This plateau rises almost abruptly against the Bangla Desh plains in the form of an elevated horst and is apparently a detached part of the Indian shield. It is delineated on its southern margin by a long E-W trending normal fault called the Dauki fault. At its eastern edge this fault joins the NW-SE striking belt of schuppen which parallel the Burmese Arc to the west. The northern edge. of the plateau is separated from the Himalayan arc by the Brahmputra Valley. In the wedge between the Himalayan and the Burmese arc and further north east of the Meghalay plateau are exposed a series of similar hills, notably the Mikir hills and the Mishmi hills. They are, however, detached from the Meghalaya plateau by alluvium filling in deep narrow grabens. This northeastern region of the India subcontinent is a part of the alpide Himalayan belt and is seismically one of the most active regions of the country. As a result of a complex convergence of the northward moving Indian plate towards the Eurasian plate along the Himalayan-Burmese arc the investigated area is probably one of high tectonic stress.

The seismicity of the area exhibits some diagnostic anomalous features. Major earthquakes are found to be preceded by a premonitory decrease in seismicity extending over periods commensurate with that suggested by the magnitude precursory time scale. This observation appears to feature consistently in respect to several $M \geq 6.7$ events in the Assam gap including the Meghalay plateau. The region is thus a most promising site for the study of the physics of the crustal processes preparatory to great earthquakes. Furthermore, the occurrence of crystalline rocks in the area offer an advantage for recording high frequency seismic signals quite clearly. 
The objective of the present investigation was to monitor microearthquake activity with a view (i) to establishing the feasibility of such investigations from the logistics point of view (ii) to determining the level of small earthquake activity and (iii) to delineating the nature and extents of active zones and their relationship with the tectonics of the area.

Accordingly, a network of 3 microearthquake stations was set up in the region in May 1979 which operated up to the end of october 1979. The locations of these stations are shown in Figure $I$ and given in Table 1.

\begin{tabular}{lllr}
\hline Name & Latitude $(\mathrm{N})$ & Longitude $(\mathrm{E})$ & Elevation \\
\hline Raliang & $25^{\circ} 28^{\prime} 03^{\prime \prime}$ & $92^{\circ} 25^{\prime} 34^{\prime \prime}$ & $1500 \mathrm{~m}$ \\
Borjori & $26^{\circ} 23^{\prime} 52^{\prime \prime}$ & $92^{\circ} 56^{\prime} 19^{\prime \prime}$ & $100 \mathrm{~m}$ \\
Burnihat & $26^{\circ} 03^{\prime} 34^{\prime \prime}$ & $91^{\circ} 53^{\prime} 19^{\prime \prime}$ & $100 \mathrm{~m}$ \\
\hline
\end{tabular}

Table 1: Locations of portable seismographs operating during May-oct 1979

\section{(4b.) Field Operations (Logistics)}

The Meghalaya plateau constitutes the State of Meghalaya of the Republic of India. It covers an area of approximately $23,000 \mathrm{~km}^{2}$ at elevations ranging from 150 to 950 meters. To its north lies the state of Assam and to the south and southwest lies Bangladesh. The smooth rolling hills formed by the weathering of ancient metamorphics and its verdant cover nourished by abundant rain make for a picturesque landscape. The capital of the state is Shillong. The mean temperatures range from $5^{\circ} \mathrm{C}$ in winter to $25^{\circ} \mathrm{C}$ in summer. The region is characterised by tropical monsoon climate with an average annual rainfall of $205 \mathrm{~cm}$.

The three station sites were selected after an analysis of the local geology and also to take advantage of the WWSSN observatory operating at Shillong. The sites were chosen at Borjori, Burnihat and Raliang. Together with Shillong 
they form a 4 station network. The diameter of the three MEQ station network is about $100 \mathrm{~km}$. and that of the four station network including Shillong about $150 \mathrm{~km}$. (Figure 1).

The stations are connected by roads. They were each manned with a technician whose duty was to maintain the instruments, change the record each day, impinge the radio time signals on the seismograms and prepare the initial station bulletins. The seismograms were collected by a supervisor going round these stations by jeep about once a month. The trip was also used to replenish stocks and supplies. This arrangement was found to be quite satisfactory for operations in India, for qualified manpower is available economically. Moreover, as the journey between stations by jeep usually takes about 8 hours and petrol costs are high, one observer going round to all the stations daily to change seismograms was not an economical mode of operation.

The signals broadcast by the National Physical Laboratory, although quite weak in the area, could however be satisfactorily used to synchronize the internal clocks of the MEQ recorders. This was done once every day. Use of selective receivers especially designed for the purpose, when made available, would greatly facilitate this task. The clock drift per day was found to be less than $100 \mathrm{~ms}$.

The three instruments used were the Teledyne Geotech Portacorder Model $\mathrm{RV}-320$, and portable short-period geophones model $\mathrm{S}-13$ working at $1 \mathrm{hz}$ natural frequency. This system has the following operating characteristics.

Recording media:

Record size:

Record format:

Pendeflection (max):

Sensitivity

Drum rotation rates:

Translation rates: smoked paper

$400 \times 600 \mathrm{~mm}$

Rectilinear

selectable $7,14,28 \mathrm{~mm}, p-p$

$15-2.5 \mathrm{~mm} / \mathrm{V}$, midband, maximum gain

$30,60,120 \mathrm{~mm} / \mathrm{min}$ switch selectable

$1,2,4,8 \mathrm{~mm} / \mathrm{rev}$ - do - 
Recordings were made of the vertical components at all the stations at a drum rotation rate of $60 \mathrm{~mm} / \mathrm{min}$ and translation rate of $2 \mathrm{~mm} / \mathrm{rev}$. In order to examine the improvement in resolution of data that might result from recording at a faster rate of drum rotation, the speed was increased to $120 \mathrm{~mm} / \mathrm{min}$ in the $3 \mathrm{rd}$ week of September. After a week however, difficult logistics problems involved in changing the smoked paper twice daily forced a return to the slower rate of drum rotation adopted earlier.

The signal to noise ratio was good at two of these sites: Borjori and Burnihat and satisfactory at Raliang.

The local logistic support for field work was provided by the Geophysics Division of the Geological Survey of India, Shillong who also provided scientists to maintain one of the stations.

In this pilot program it was important to determine how much down time we had to expect in this area which was considered mistakenly as inaccessible by some. We found that the three station array was operated with a $20 \%$ down time. This was calculated in the following manner: All days for which each instrument was in the field were summed. This sum of 425 days is called the number of station-recording days. Then we summed all days during which an instrument was not operative, regardless of the reason for failure. If during the same day two stations were out of operation 2 down-days were counted. The total number of down-days was 87. The number of down-days and their distribution can be estimated from Fig. 2. In this histogram of recorded microearthquakes the days with zero records are generally those when the instrument was down. The seismicity was so high that there were almost no days where no earthquakes occurred. 
The experience gained in the five and a half month continuous operations in the area has confirmed our earlier conviction that such networks can be established and operated successfully in this area.

Furthermore, the prolific seismic activity monitored here suggests that much can be learned about the physics of earthquake processes in this area.

Finally, it may be added here that the North Eastern Council, which is a body responsible for planning the overall development of the region and its environment, has recently formed a Committee for the Earthquake Risk Evaluation of the area with the specific objective of advising the states' Civil Administration as to how to effectively use the results of various scientific activities in the region, connected with earthquake prediction towards the design of appropriate precautionary systems so as to minimize the hazards that the region may suffer due to a large earthquake.

\section{(4c.) Research Programs Conducted by other Agencies}

A most welcome outcome of the presently funded research project has been the formulation and initiation of a wide-ranging prediction and risk evaluation program in the Assam region by Indian scientists. For, whilst the seismic susceptibility of the area has long been recognized leading to considerable geological and geophysical investigations, the current intensity and mobiliza- . tion of these efforts within the country can be traced to the appearance of our paper drawing attention to the seismic gap in Assam and exposing the rationale for the project under discussion. 
The Indian program envisages a multi-institutional coordinated effort towards monitoring the microearthquake activity, ground deformations and anomalous changes, if any, in the gravity and magnetic fields as well as variations in resistivity, seismic wave velocities, radon content and well water levels. These are briefly outlined below.

\section{i) Microearthquake studies}

The various Indian. organizations involved in monitoring the microearthquake activity in the northeastern region have agreed to concentrate their efforts in its different sectors with the provision of making conjunctive use of their data through exchange of information. These are as follows:

a) Department of Earth Sciences University of Roorkee

and

the Geological Survey of India Meghalaya Plateau and Mikir Hills.

N.E. Division

b) India Meteorological Dept., Department of Earthquake Engineering University of Roorkee The area covered by the and Bramhaputra Valley.

the Central Water Commission

c) The National Geophysical Research Institute, Hyderabad

and

the Regional Research Laboratory Jorhat

Naga thrust, the south and the southeastern part.

ii) Levelling

The Survey of India carried out high precision levelling surveys along an almost $\mathrm{N}-\mathrm{S}$ profile traversing the Meghalaya Plateau from Gauhati to Silchar over a decade ago. According to the integrated plan coordinated by the Geological Survey, they propose to reoccupy this profile and also carry out a similar survey along an E-W profile beginning from Tura at the western edge of the plateau to Garampani at its eastern flank. Additionally, they 
would provide high precision location coordinates for gravity and magnetic profiles to be carried out by the Geological Survey along these two intersecting profiles and elsewhere.

iii) Radon measurements, resistivity, gravity, magnetic and refraction seismics for monitoring velocity variations

The Geophysics Division of the North Eastern Division of the Geological Survey of India would be carrying out these surveys at anomalous sites deineated on the basis of seismic activity.

iv) The North Eastern Hill University which is located at Shillong is also in the process of formulating a few research programs to involve its faculty in this venture, particularly in studying anomalous magnetic variations, premonitory animal behaviour, and social aspects of earthquake prediction.

The project funded by the U.S.G.S. is an integral part of the above outlined multi-institutional program. We expect that our project will be one of the leading elements in the large co-operative project, providing stimulation to some of the tasks executed by others.

\section{Data Analysis}

Examples of seismograms are shown in Appendix A. The selection of these. examples was made at random with the constraint that all three instruments had to have produced a record. Most of the seismograms in Appendix A show 24 hour periods. Even though the Xerox copies of the mostly black appearing photographs are poor copies, it is clear from Appendix A that a large number of earthquakes were recorded. Tickmarks on the seismogram traces appear every 10 sec. Most of the signals lasted for several $10^{\prime}$ s of seconds, many up to 100 seconds. This shows that the magnitudes of these events are fairly large. 
For a general measure of the seismicity rate which one can record in this area we compiled histograms for the three stations. Figure 2 shows the daily number of local earthquakes recorded by the three stations. BOR was installed first, Burnihat last. BUR showed the largest seismicity rate. This was probably due both to its good signal to noise ratio as well as to its proximity to an active area. The station RAI with the poorest signal to noise ratio recorded the least number of events. However, at all three stations very large numbers of shocks were recorded. On the average the rate was approximately 20 events per day.

Arrival times were read for earthquakes with S-P times of generally less than $40 \mathrm{sec}$. That is, sources with distances of more than about $300 \mathrm{~km}$. from the array were excluded from the analysis. Preliminary readings of P-arrival times, s-arrival times, maximum amplitude and signal duration are tabulated in Appendix B. The analysis of this data set is not terminated; it continues under the current U.S.G.S. contract to study Seismic Risk in the Assam area. The bulletin-data in Appendix B are not yet completely purged of errors. We are now in the process of final data revision for this first set of approximately 400 events. This preliminary bulletin only covers the period mid-June to mid-August. The arrival times for events which occurred during mid-August through October are being read now.

In its present preliminary form the bulletin contains almost no Shillong. readings. Because of its lower frequency response characteristics sHI does not record many of the local signals clearly measurable at the portable seismographs. Nevertheless we will examine the SHI records for reading arrival times for the larger events and for those which occurred close to Shillong. 
In addition we hope that complementary data from microearthquake arrays operated by other Indian institutions during the fall of 1979 may become available. If this should be the case many of the events which now cannot be located may become locatable by joining data from different networks.

Preliminary locations have been computed for some of the better recorded earthquakes. These data are summarized in Appendix C. However, we must emphasize that these are only approximate and preliminary locations. Improved locations will be calculated when (a) more arrival times become available for these events (from SHI and possibly other portable stations), and (b) when we know the crustal velocity structure better. Since we have no specific information on the local velocity structure we have used the model below which is appropriate for the Swiss Alps and therefore can be expected to be close to structure in the Assam area.

$\begin{array}{cccc}\text { LAYER } & \text { VELOCITY } & \text { DEPTH } & \text { THICKNESS } \\ & \text { KM/SEC } & \text { KM } & \text { KM } \\ 1 & 4.0 & 0.0 & 1.0 \\ 2 & 6.0 & 1.0 & 24.0 \\ 3 & 6.7 & 25.0 & 20.0 \\ 4 & 8.1 & 45.0 & 1000.0\end{array}$

In order to estimate where the main centers of seismic activity are located we included in the analysis epicenters outside the array. We realize that locations outside of a tripartite network are very inaccurate and calculated depths nearly meaningless. Nevertheless we can say from which general areas many signals come, an important piece of information to plan the continuation of this program with a larger array. The hypocenters (Appendix C, Figure 1) were computed with the HYPOELIPSE program. The quality of location is generally 
poor because only three stations were recording, and the arrival times were read mostly to the nearest 0.5 seconds only. A few of the solutions are of C - quality. With the expected additional data from SHI some solutions will be improved.

The centers of activity are within our array near BOR, near Tezpur north of the array, and along the Burmese thrusts SE of the network. NE in the rest of the Assam gap very few earthquakes occurred. This agrees with the observation of Khattri and Wyss (Appendix D) who pointed out that the eastern part of the Assam gap appears to have conspicuously low seismicity.

Even though the teleseismic locations are even less accurate than the ones by tripartite array, it appears that the presently active volumes correspond to the locations of past larger earthquakes. In order to better locate the strong activity near $B O R$ and north of it we will need to place a station at a location north of the word Tezpur on the map (Figure 1). This is especially necessary because some of the sources around Tezpur appear to be located at depths below the crust. With an expanded array (as funded by the U.S.G.S.) we will be able to locate a fairly large number of earthquakes within the array and obtain reliable depths and composite focal mechanisms for them.

The contrast between the high and low level of seismicity in the western and eastern part of the Assam gap (Figures 1 and 3) may be interpreted as pointing to a high stress asperity adjoining to a volume of seismic quiescence. Such a pattern was found in the detailed study of the Hawaii earthquake, and the same pattern is suggested by the Kurile island seismicity (for both these cases see our report on the U.S.G.S. sponsored study of "Seismicity Patterns Before Large Earthquakes"). In the Meghalaya area the observation 
period is much too short as yet to allow any firm conclusions, but the continuation of this study will be important to establish background seismicity and to delineate seismicity anomalies.

\section{Conclusions and Recommendations}

1. Seismic quiescence appears to precede earthquakes of magnitude larger than 6.7 in the Assam area.

2. Seismograph arrays can be operated on the Meghalaya hills, and this is best done by employing an operator for each station.

3. The seismicity is high in the general area of the Himalayan arc, the Burmese arc and in between. On the average 20 earthquakes per day were recorded within about $400 \mathrm{~km}$ from the center of our tripartite array. With this array we were able to locate about 25 events per month within the area which will be covered by the permanent network starting spring 1980 . In addition many events were recorded which could not be located. We expect that with the larger network we will locate approximately 2 events per day within the array, and about an equal number in the vicinity of the array.

4. The low seismicity rate in the eastern part of the Assam gap provides a remarkable contrast to the rate in the western part.

5. A comprehensive Indian program of earthquake prediction research in the area was launched.

6. We recommend that U.S.G.S. support for the Meghalaya seismic array and for the seismicity analysis of the area be continued. The capabilities of this network will be improved in the 1980 study phase so that precision timing of phase arrivals to less than 0.1 seconds will be possible, and so that focal mechanism solutions can be obtained. The support of this project is very important, because we still believe that a large to great earthquake may 
occur here during the lifetime of the array, and because this project is the leading component in a large integrated program. 
Financial Statement

Budget

Salary

Fringe Benefits

Operating Expenses

Travel

Permanent Equipment

Indirect Costs

TOTAL
$\$ 2370$

298

4627

1900

21545

780

$\$ 31500$
Actual

$\$ 2633$

331

2875

1954

22701

869

$\$ 31363$
Balance <Deficit>

$\$<263\rangle$ $<33>$

1752

$<54>$

$\langle 1156\rangle$

$<89>$

$\$ \quad 157$ 
Figure 1: Map of Northeastern India showing major thrust zones and the outlines of the metamorphic shield fragments of the study area. Open circles mark historic earthquakes, solid dots show preliminary locations obtained in this study. Seismograph stations are shown by $\boldsymbol{h}$.

Figure 2: Histogram of all earthquakes recorded at the three portable field seismographs with position given in Figure 1 and Table 1. Days of no records are those where the instruments were not operating.

Figure 3: Epicenter map including 122 of the 124 events for which preliminary locations have been computed, including those events too far from the network to fit on Figure 1. Small differences in locations here and on Figure 1 are due to changed weights for the phase in the location program. The Assam gap and the great thrusts of the Himalaya and of Burma are sketched in schematically. Seismograph locations are marked by stars. 


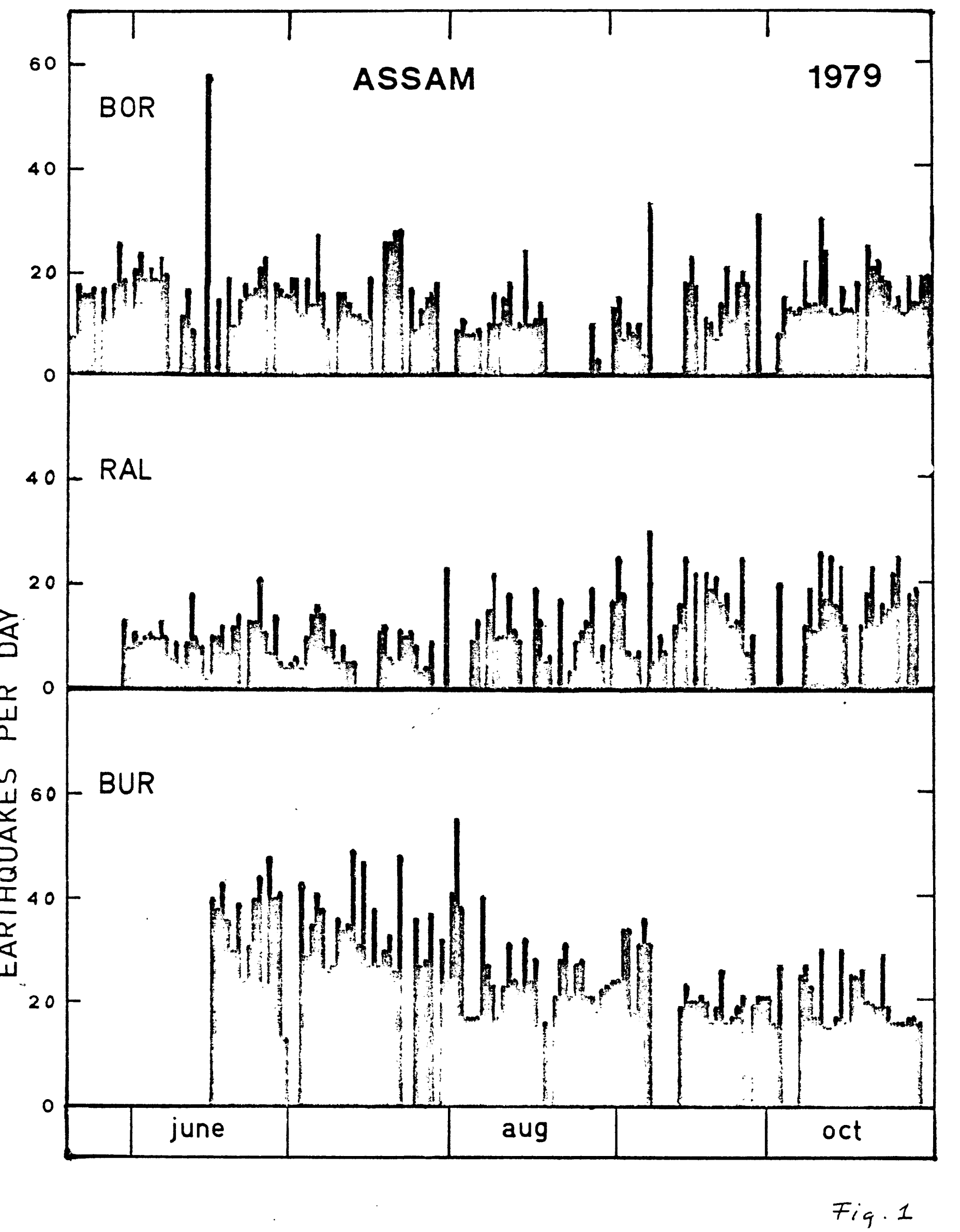


INDIA

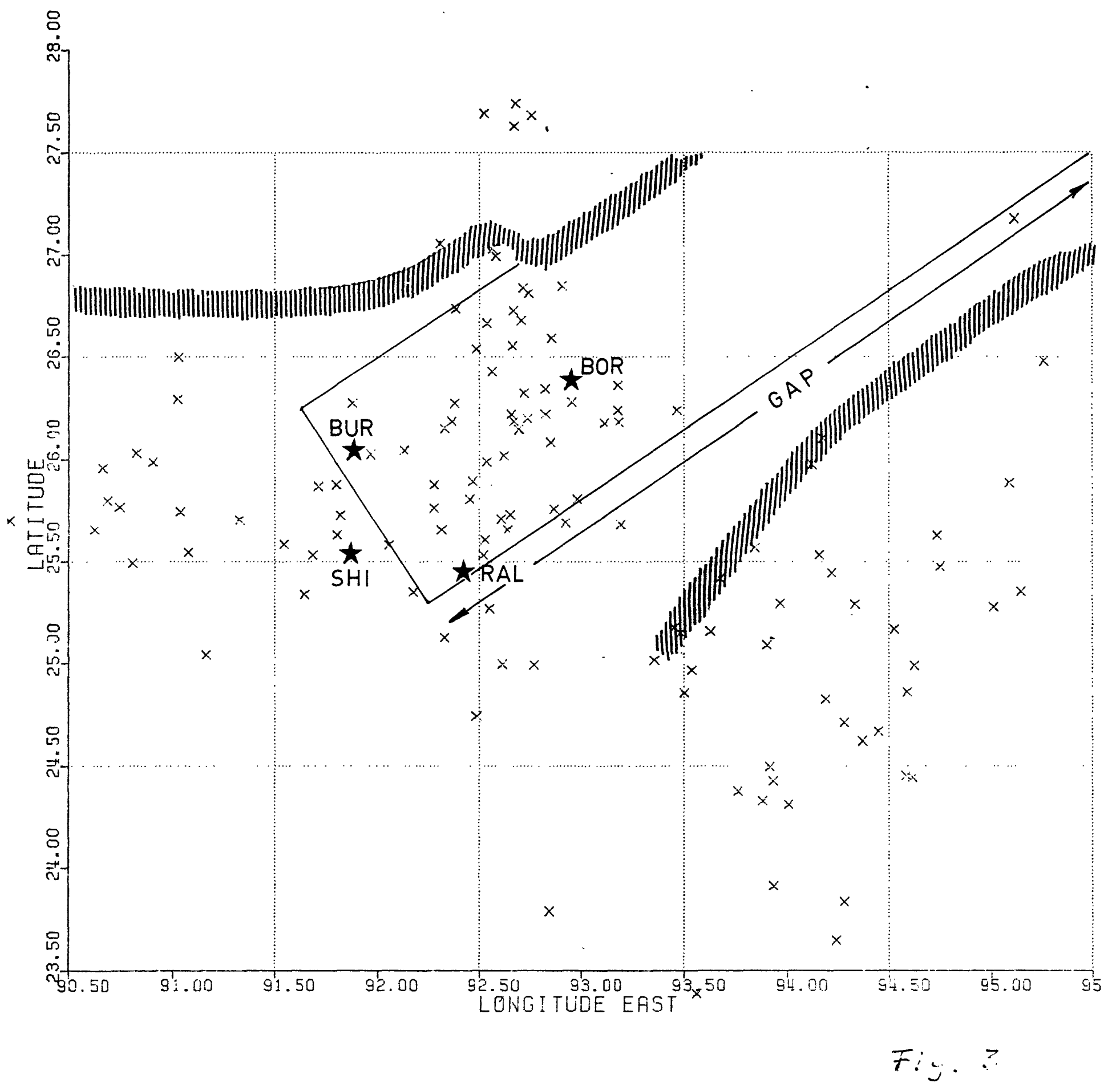


APPENDIX A

14 pages

Examples of seismograms at the three stations BOR, RAL and BUR for 9 arbitrarily selected days of fall 1979. The dates which appear as headings give day, month and year. 


\section{BÚR}
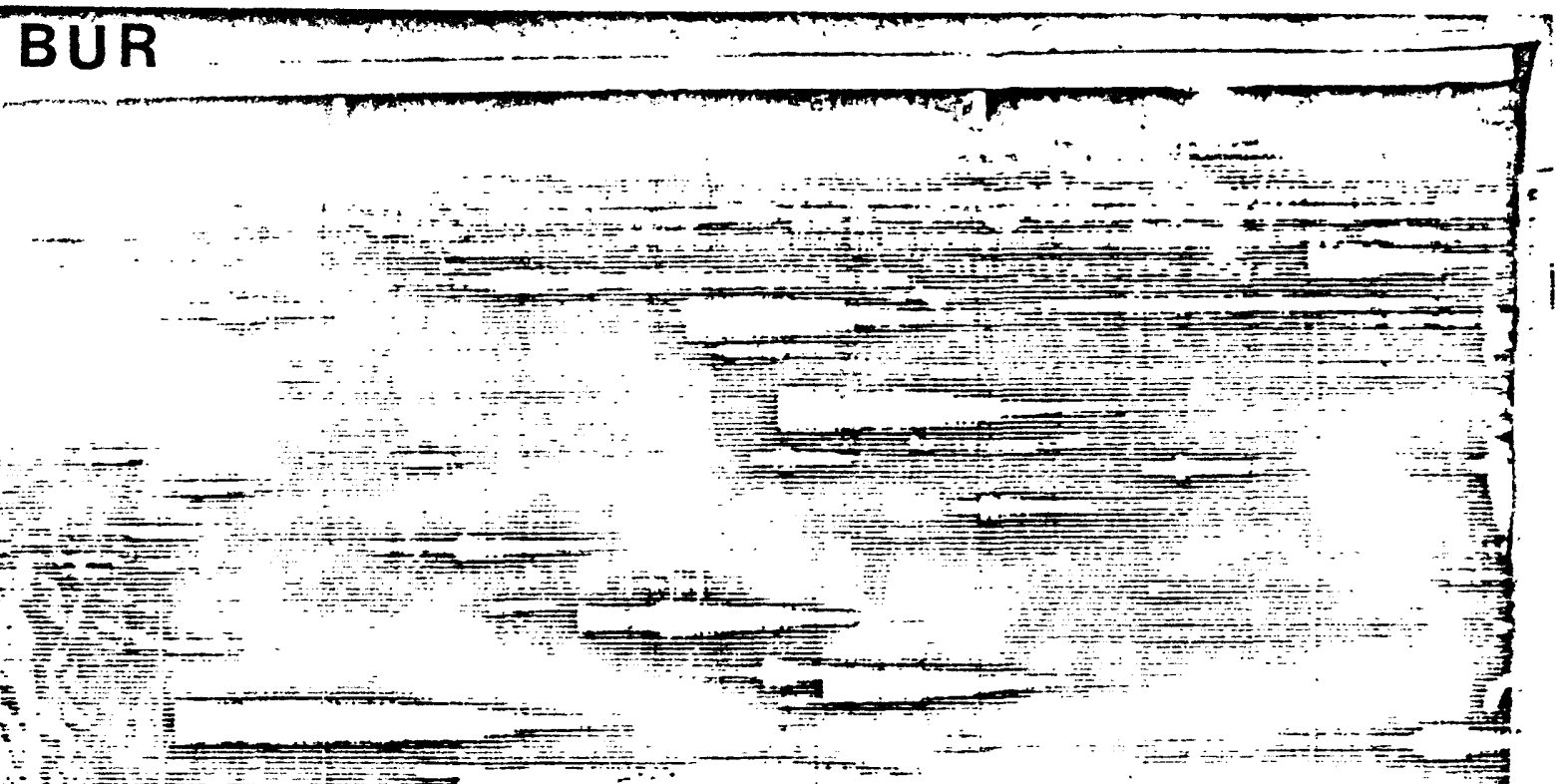

No

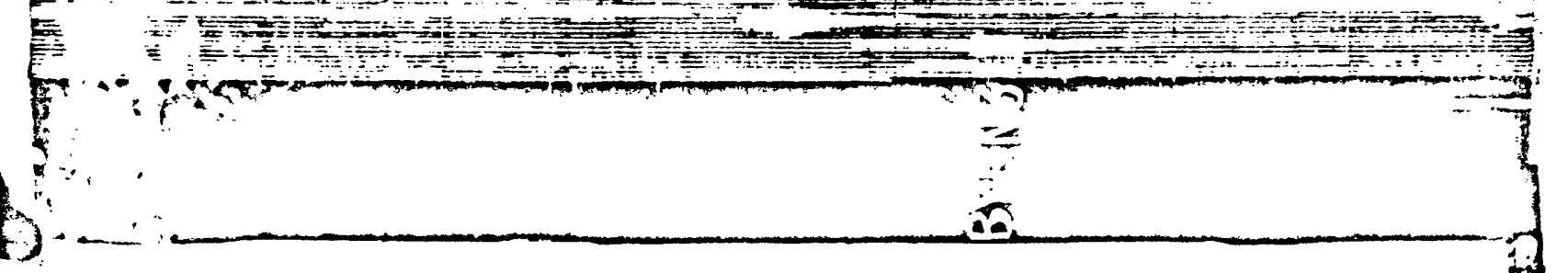

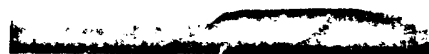

\section{9}

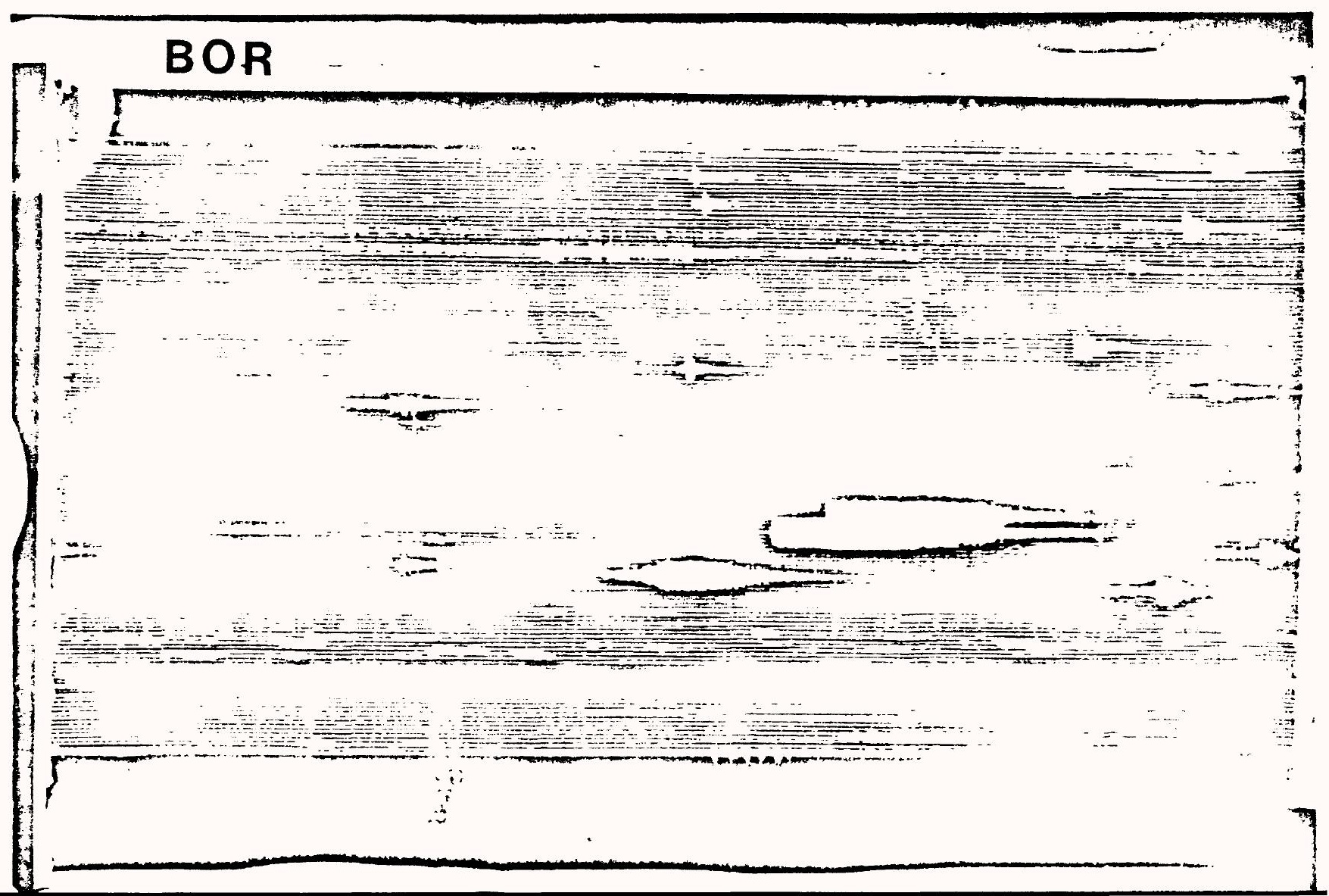



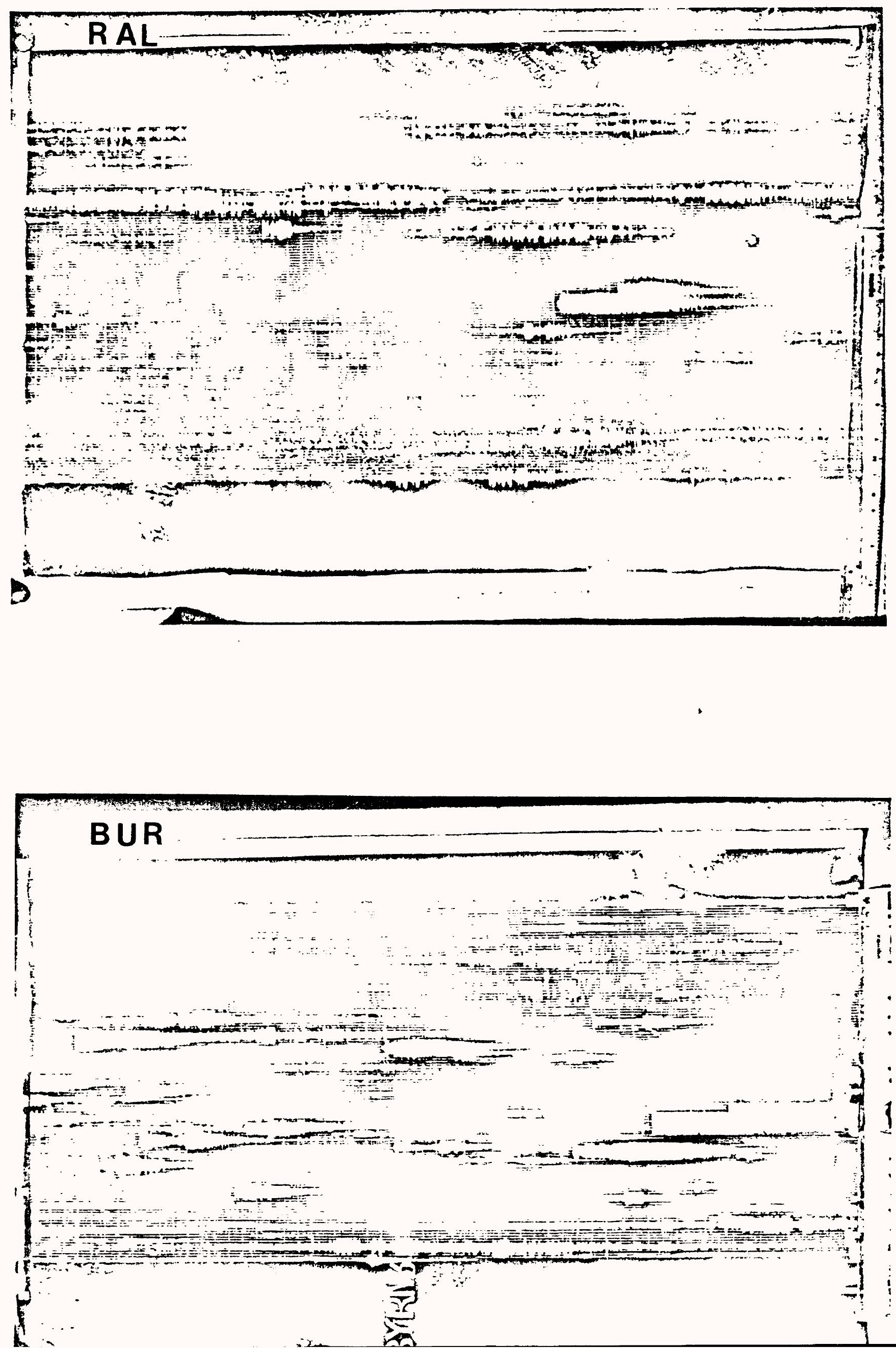


\section{$\begin{array}{lll}9 & 8 & 79\end{array}$}
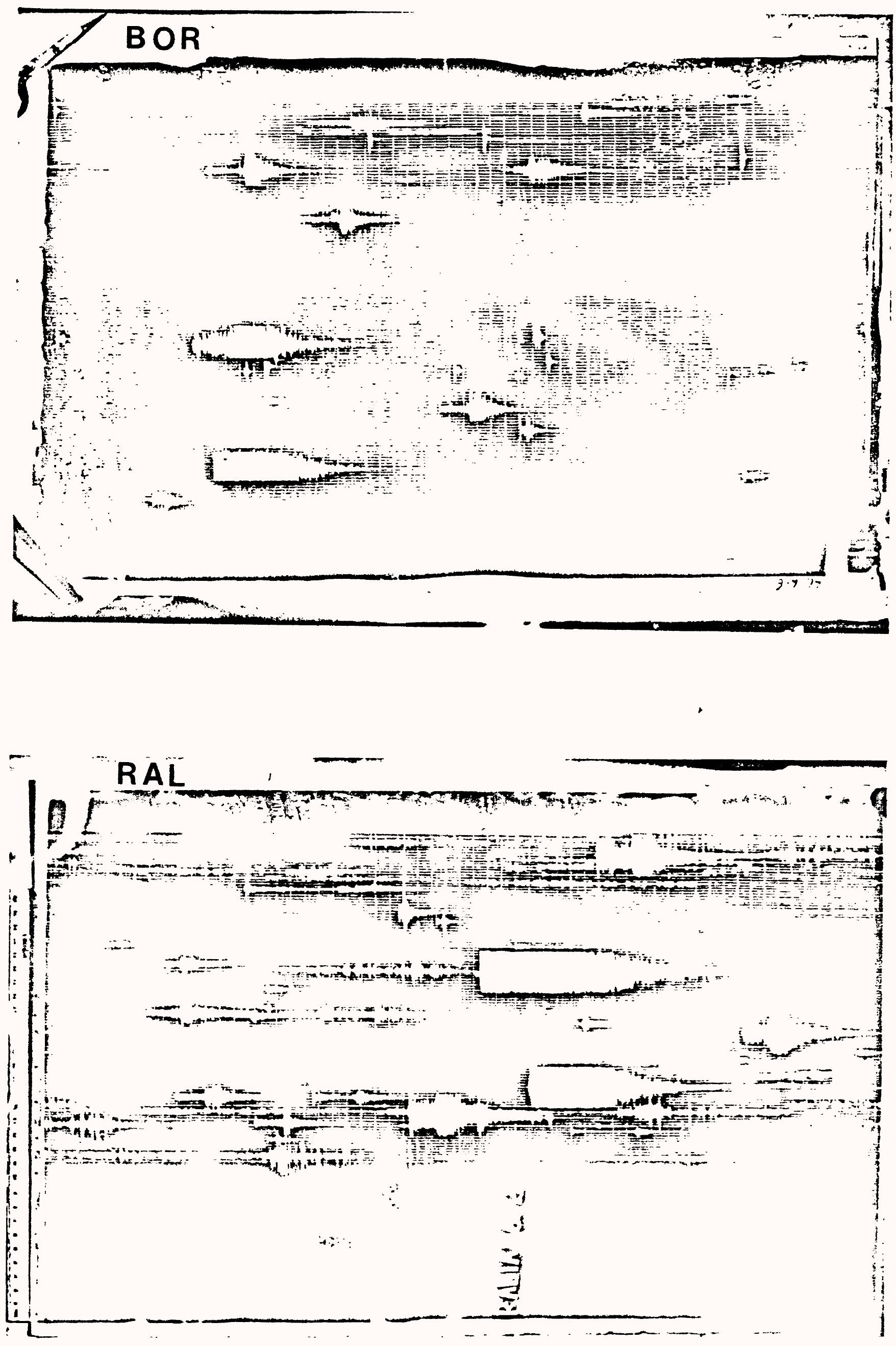


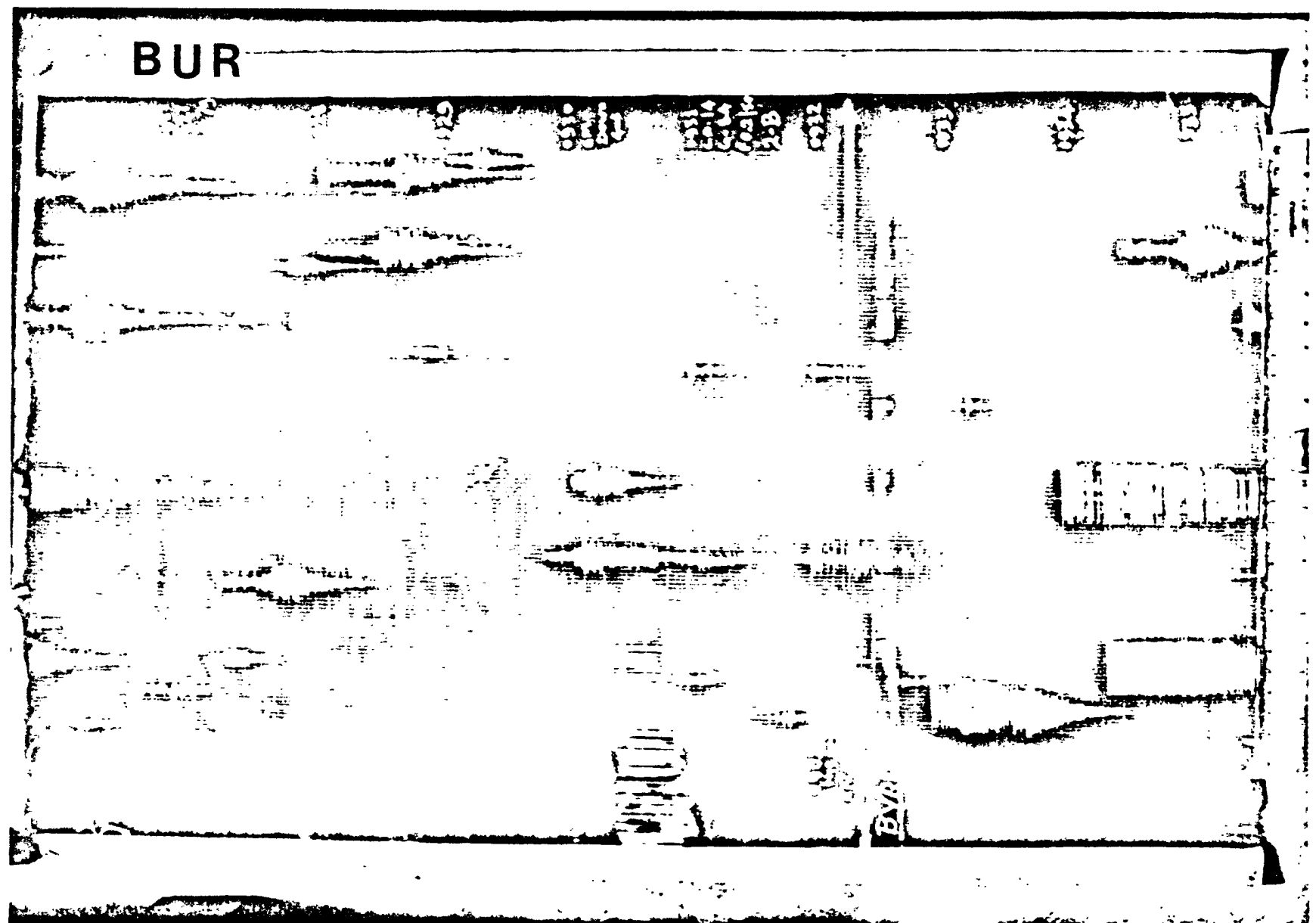

$\begin{array}{lll}15 \quad 9 & 79\end{array}$

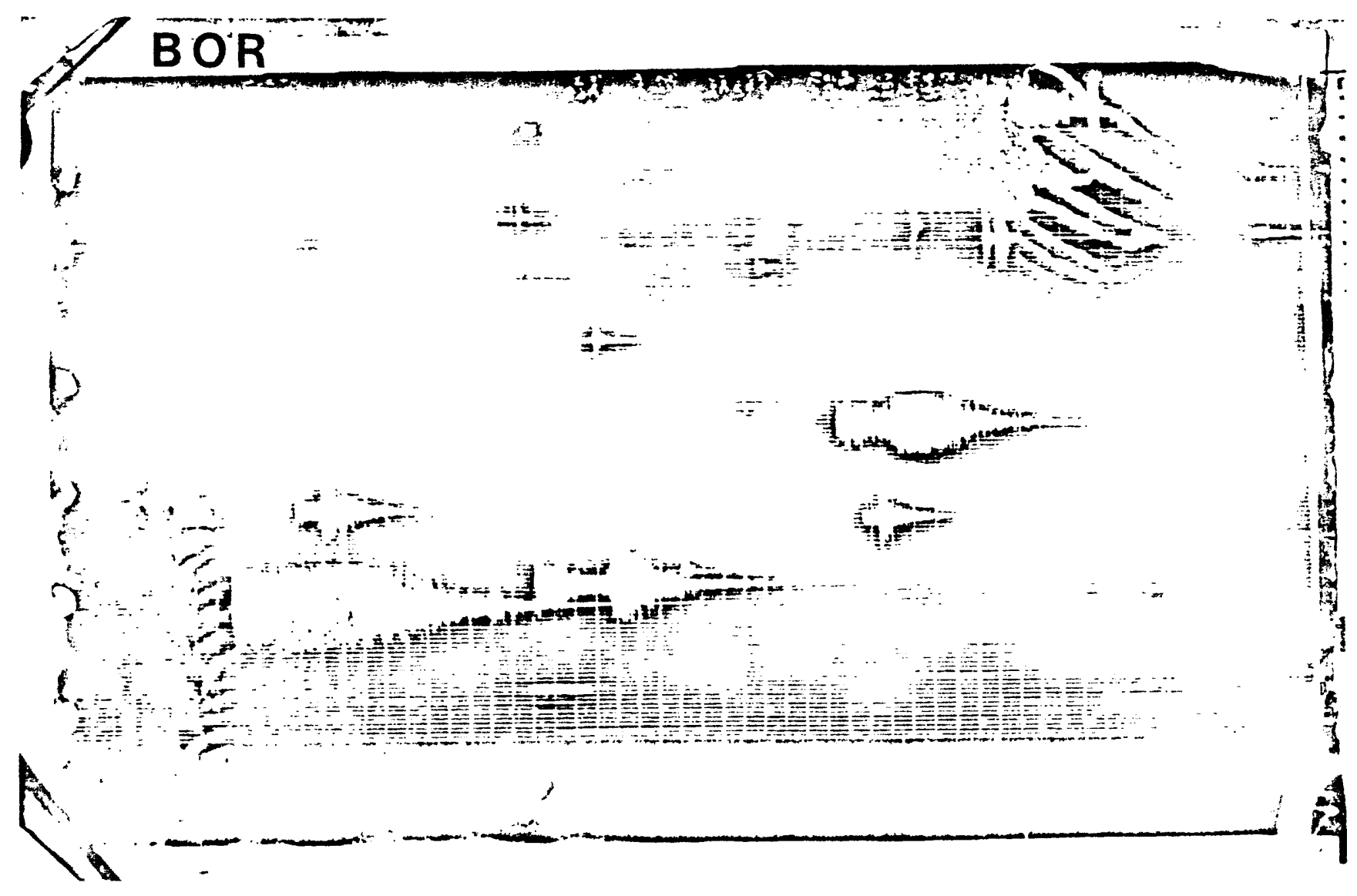



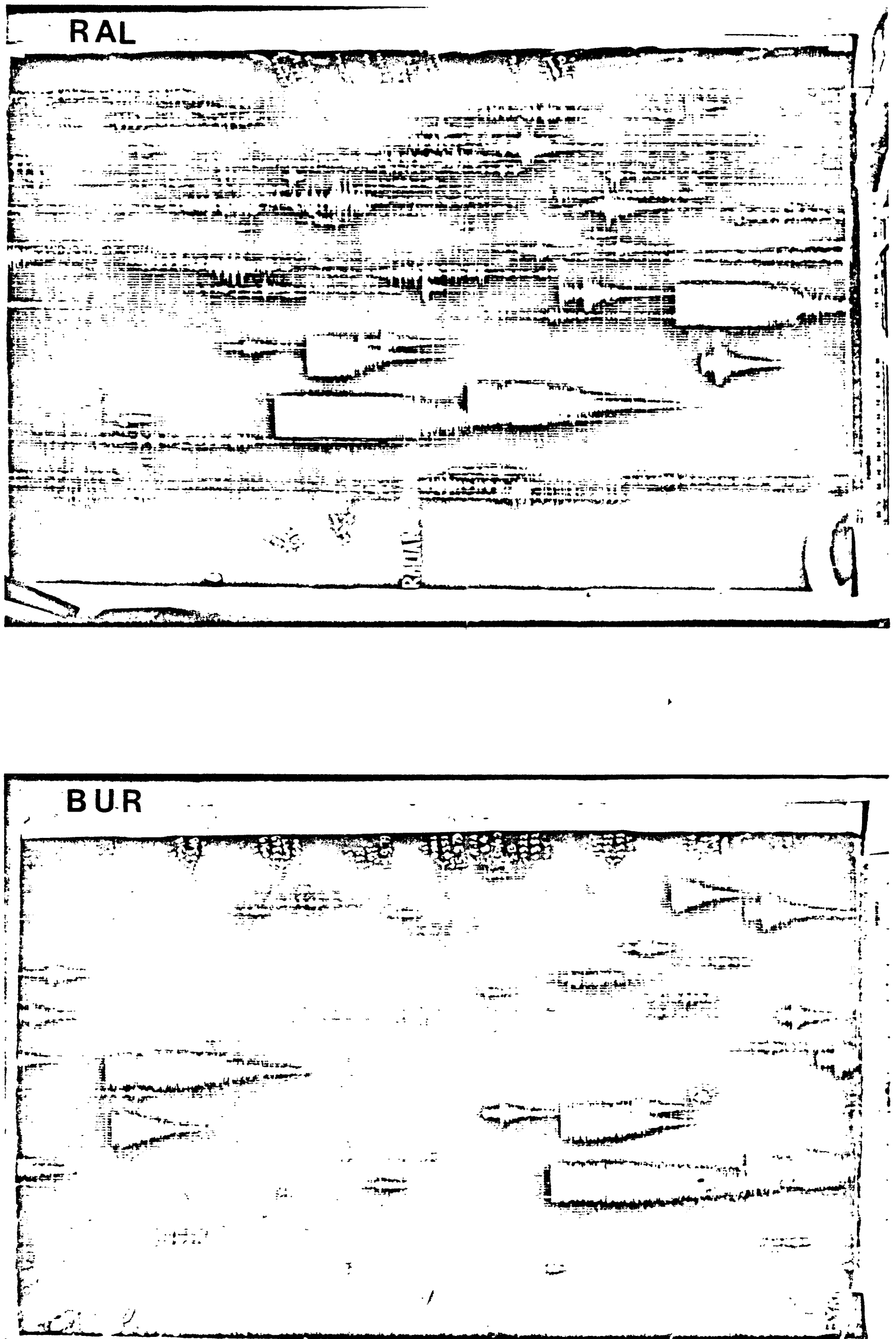


\section{$\begin{array}{lll}19 & 9 & 79\end{array}$}
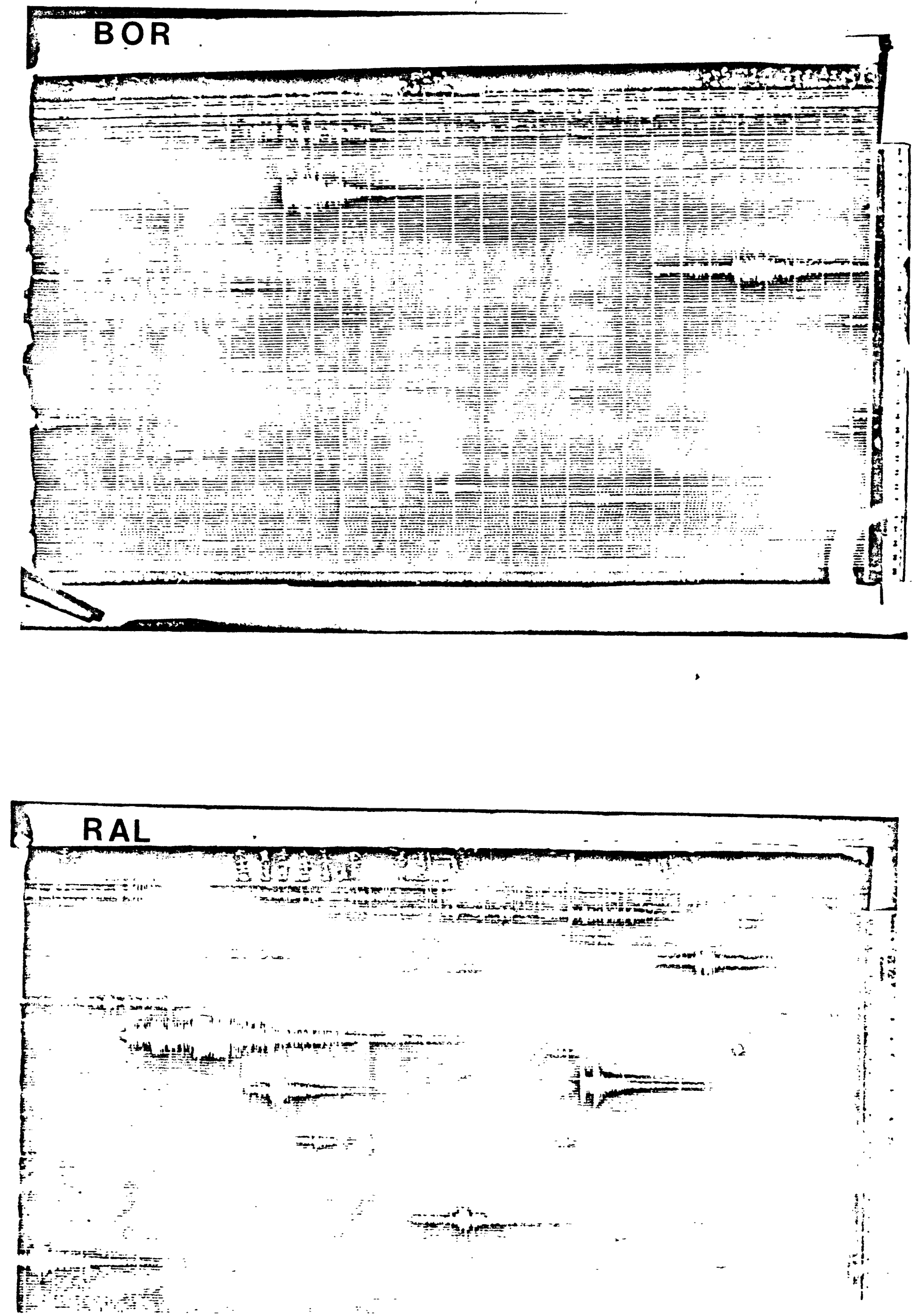


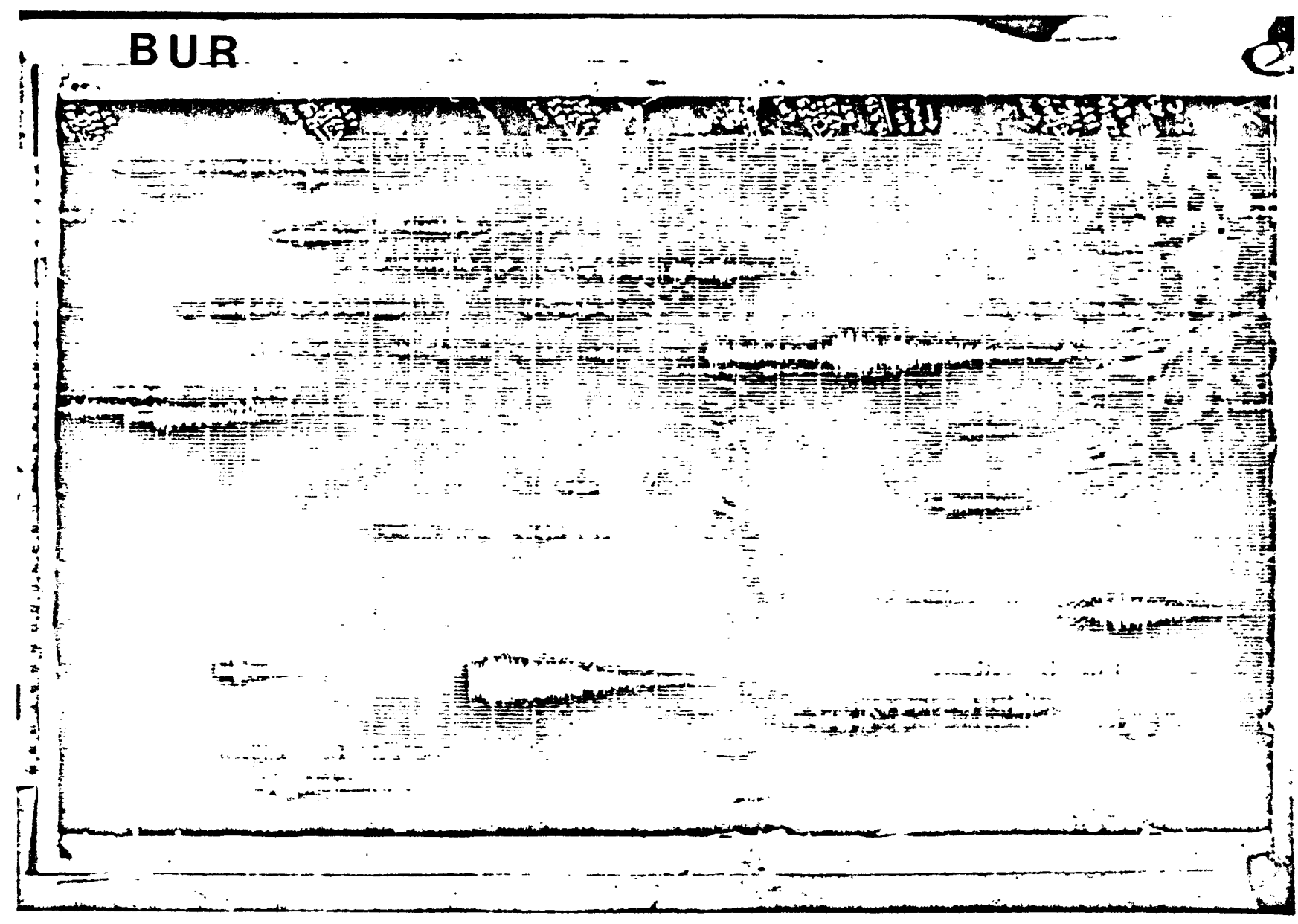

\section{9}

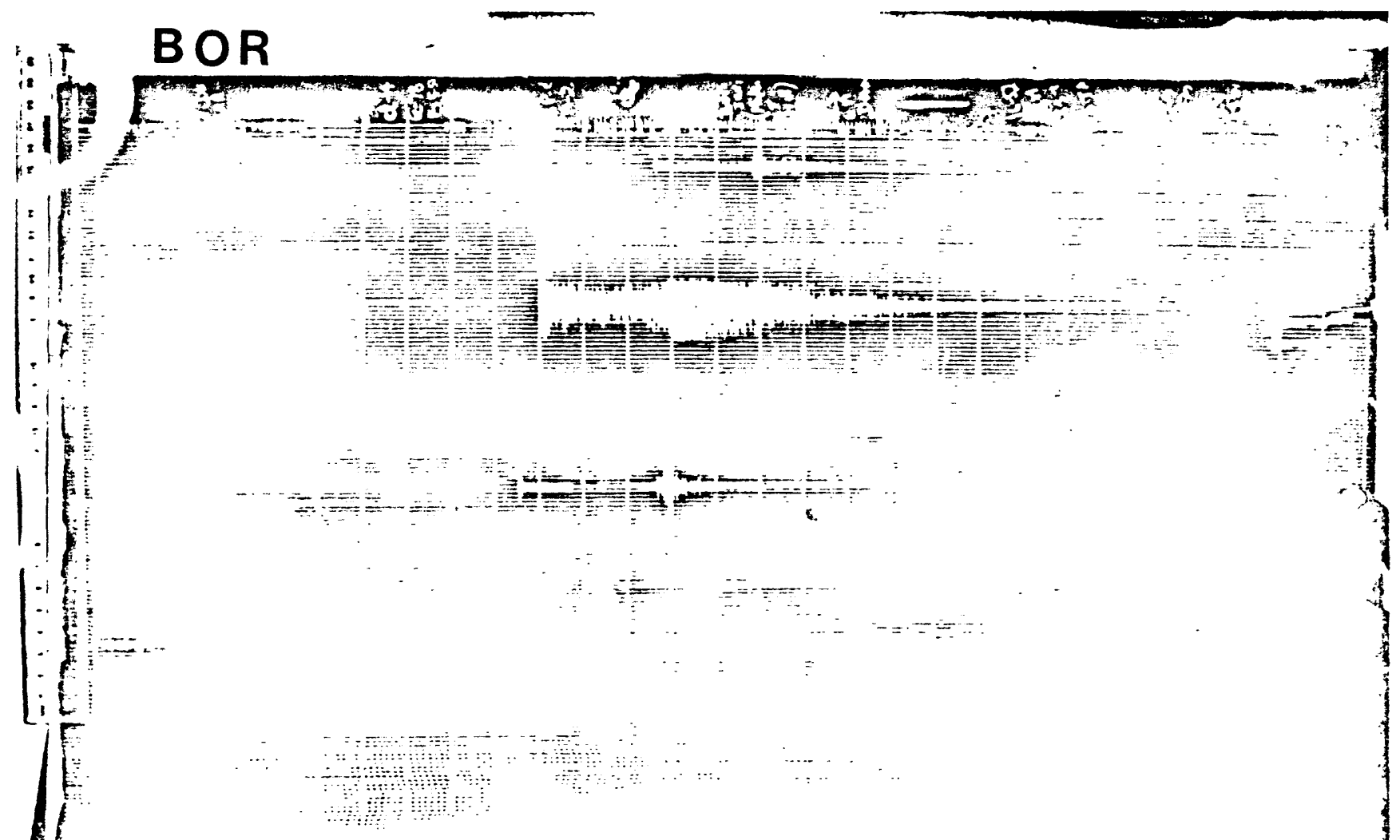



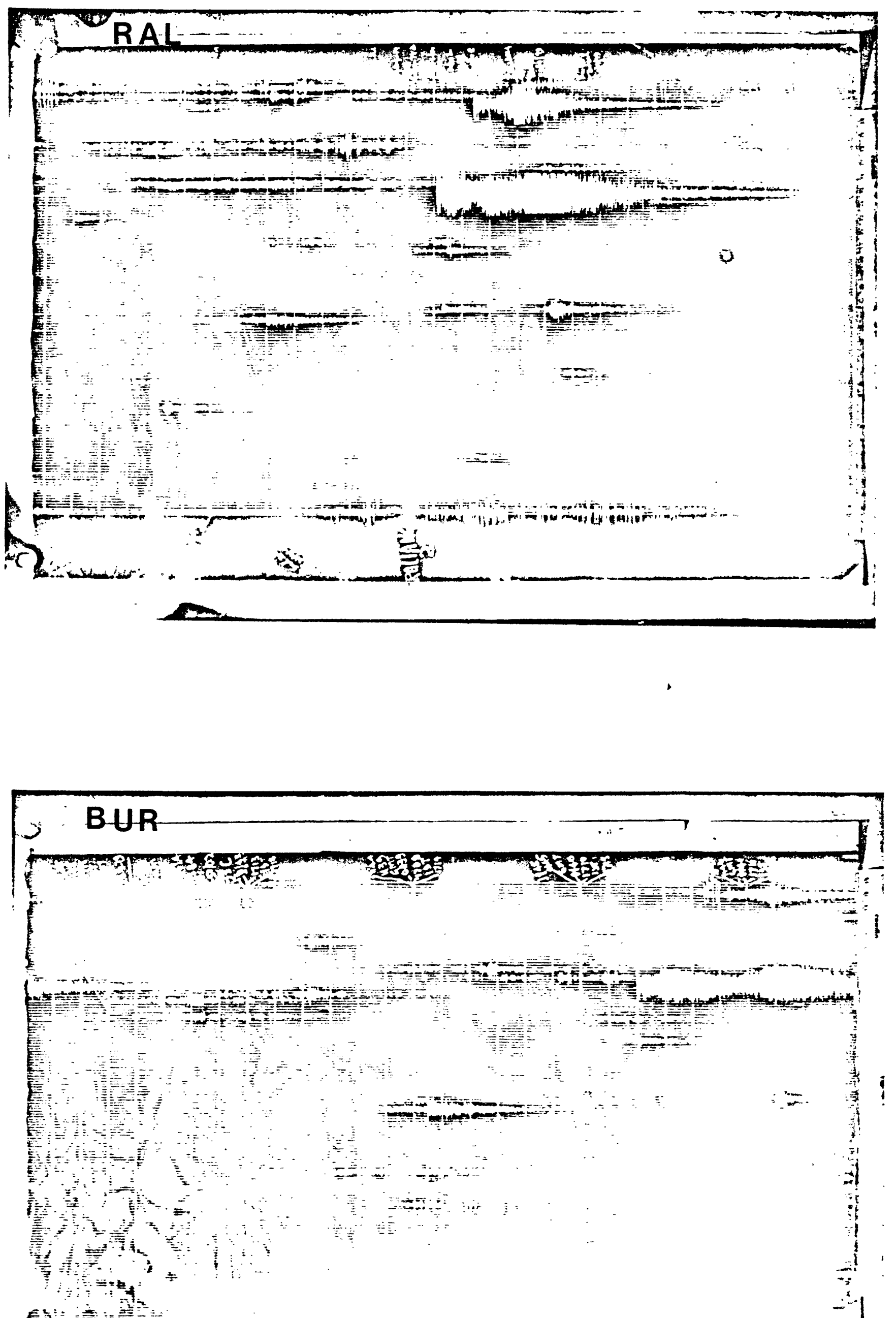


\section{$\begin{array}{lll}21 & 9 & 79\end{array}$}
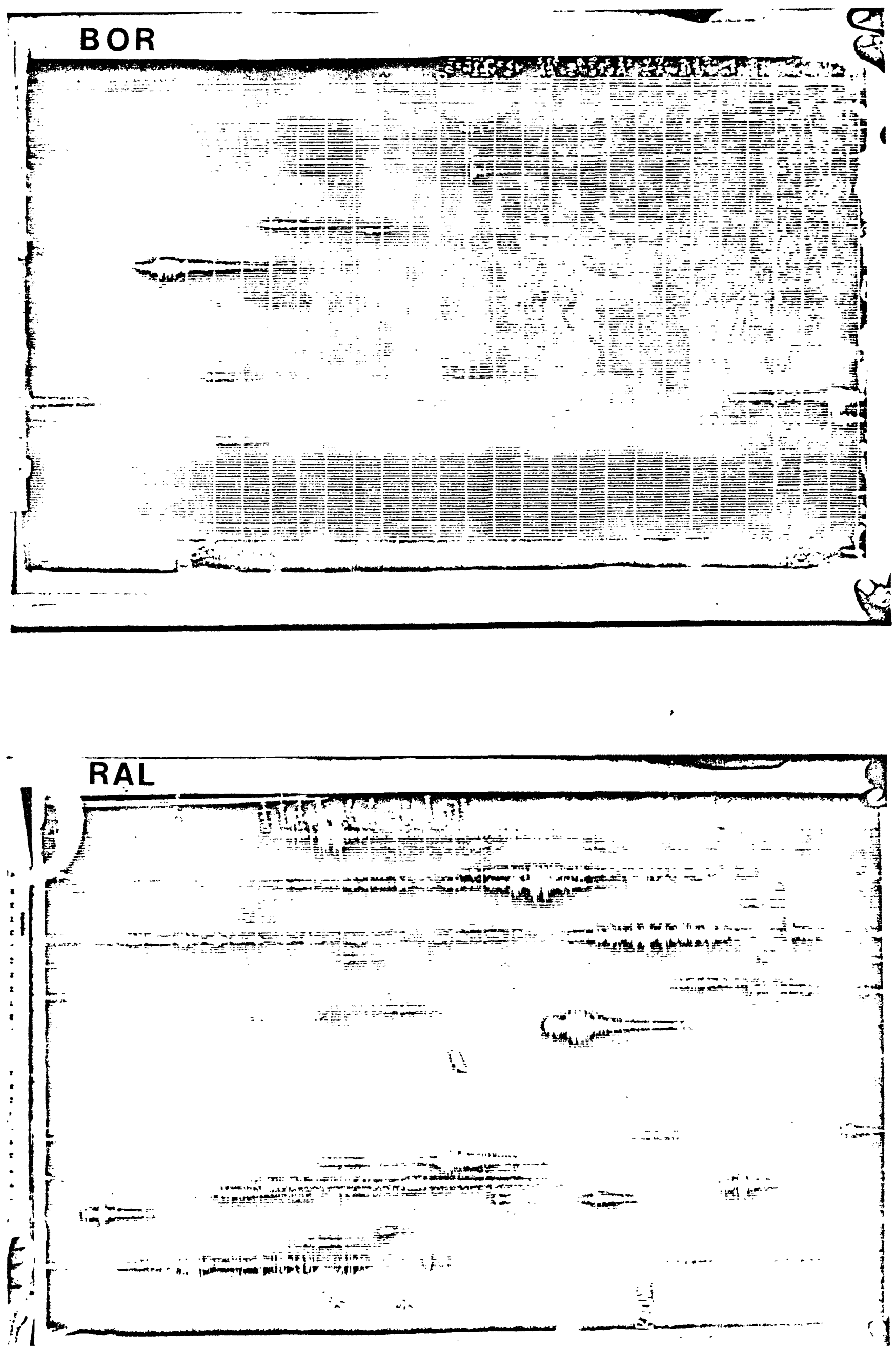


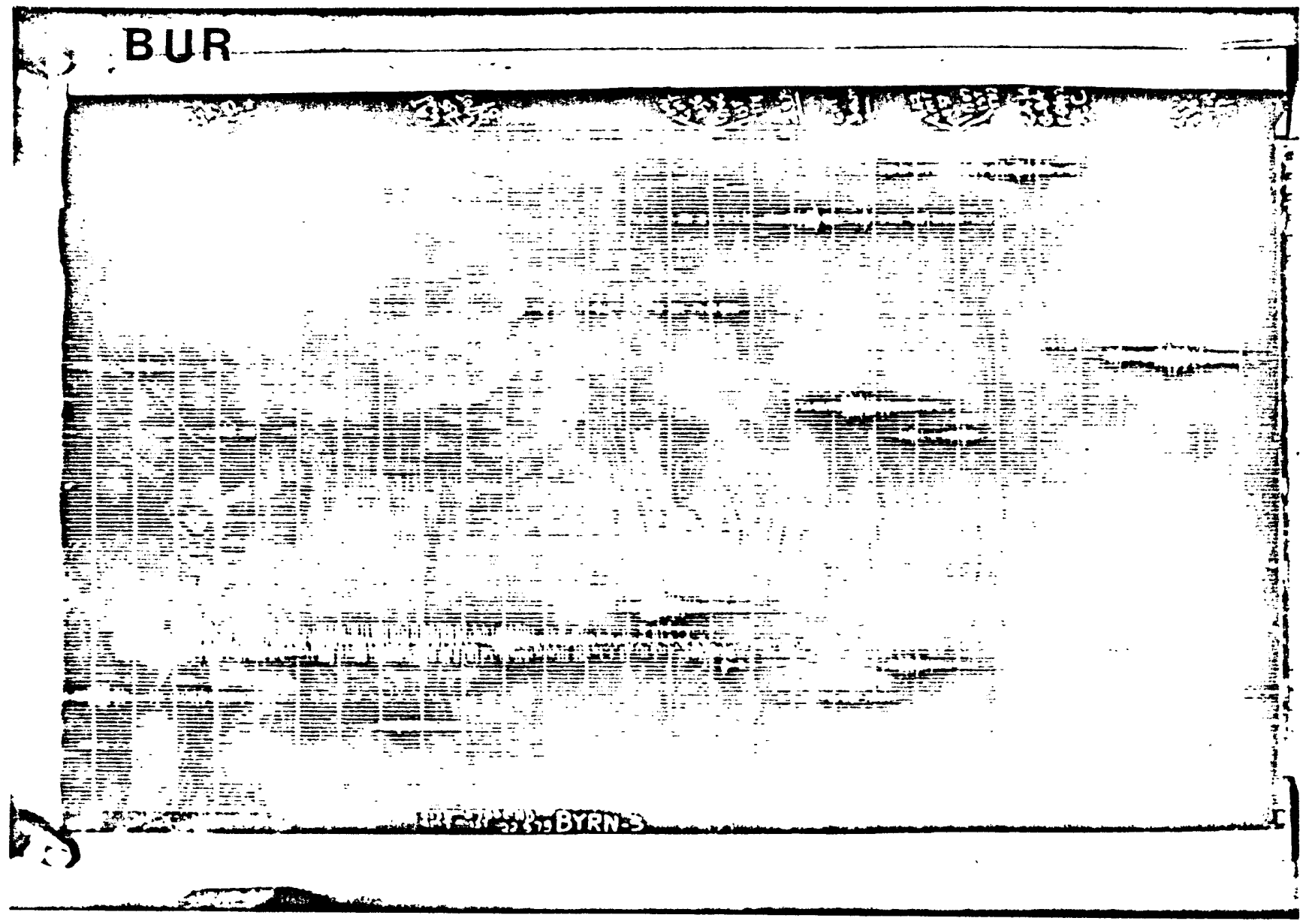

\section{$\begin{array}{lll}8 & 10 & 79\end{array}$}

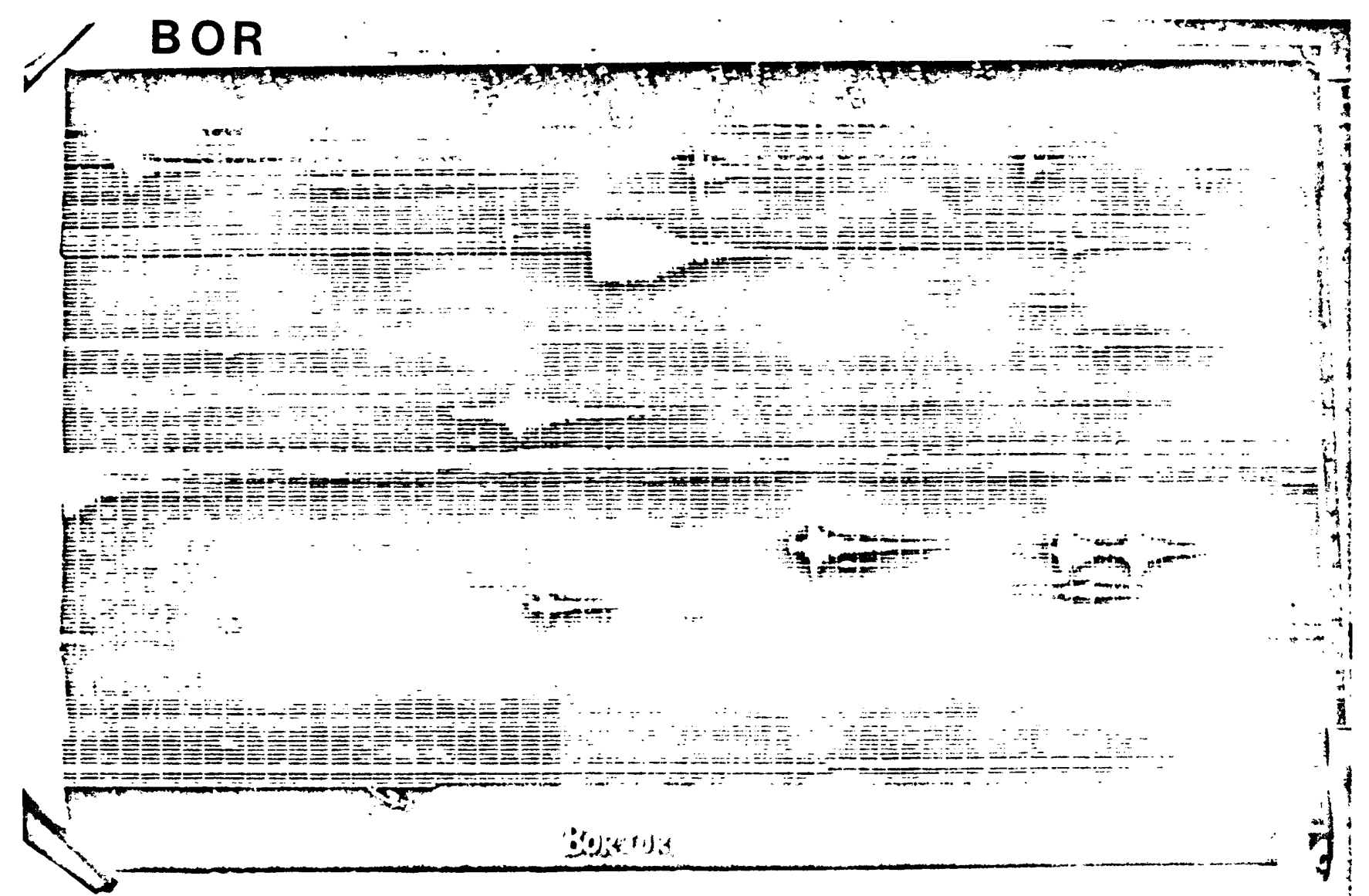




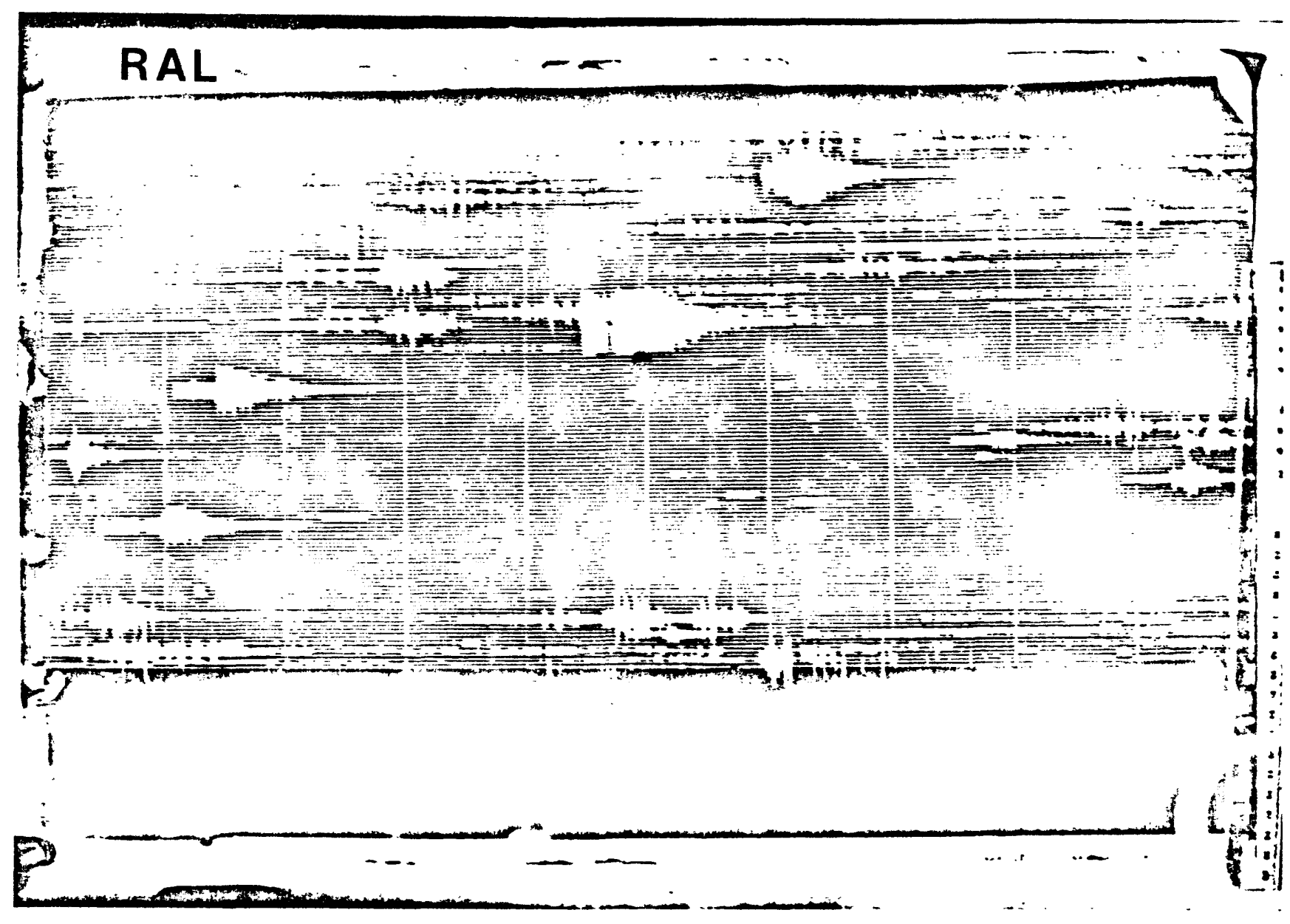

,

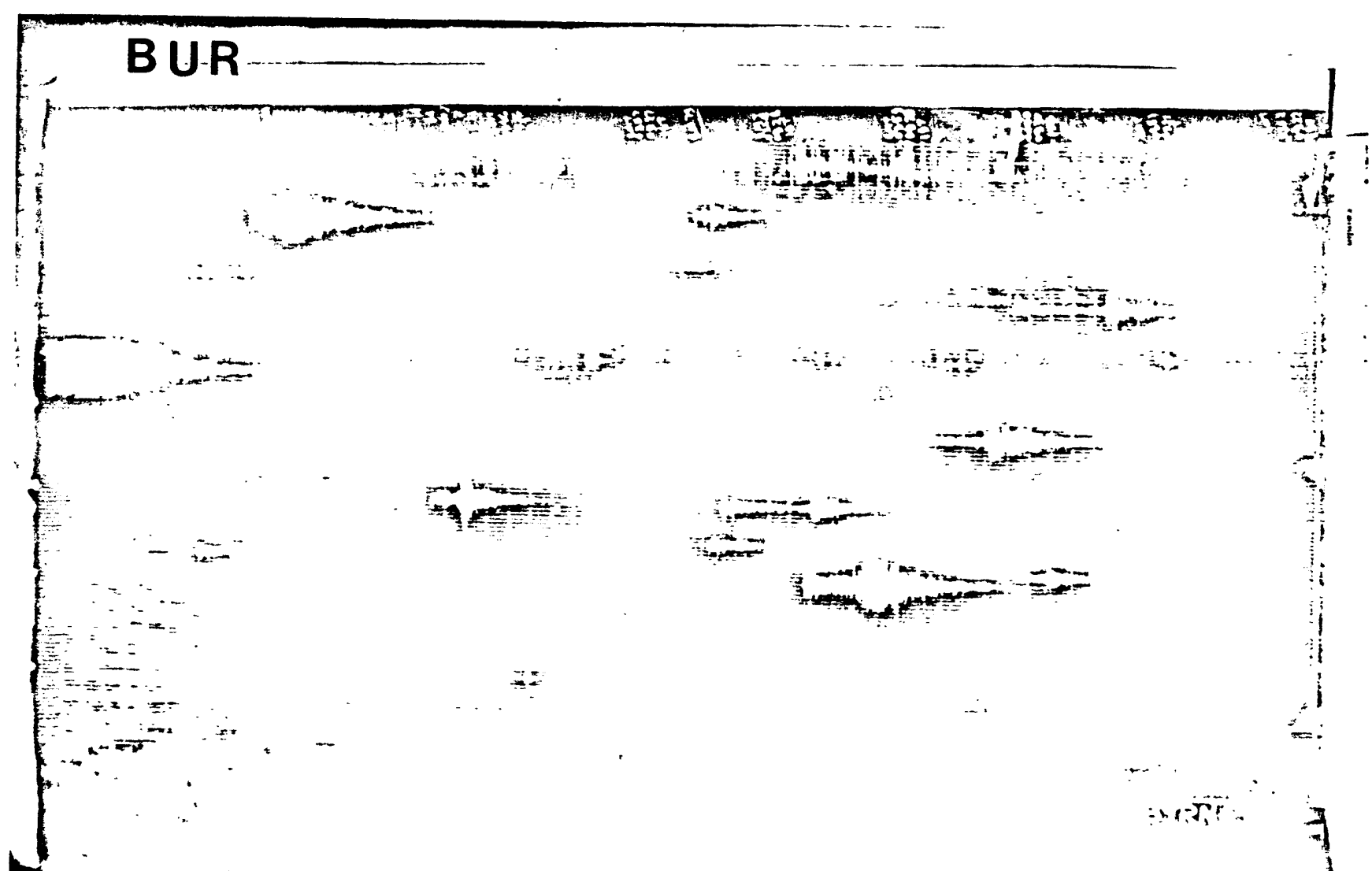




\section{$\begin{array}{lll}17 & 10 & 79\end{array}$}
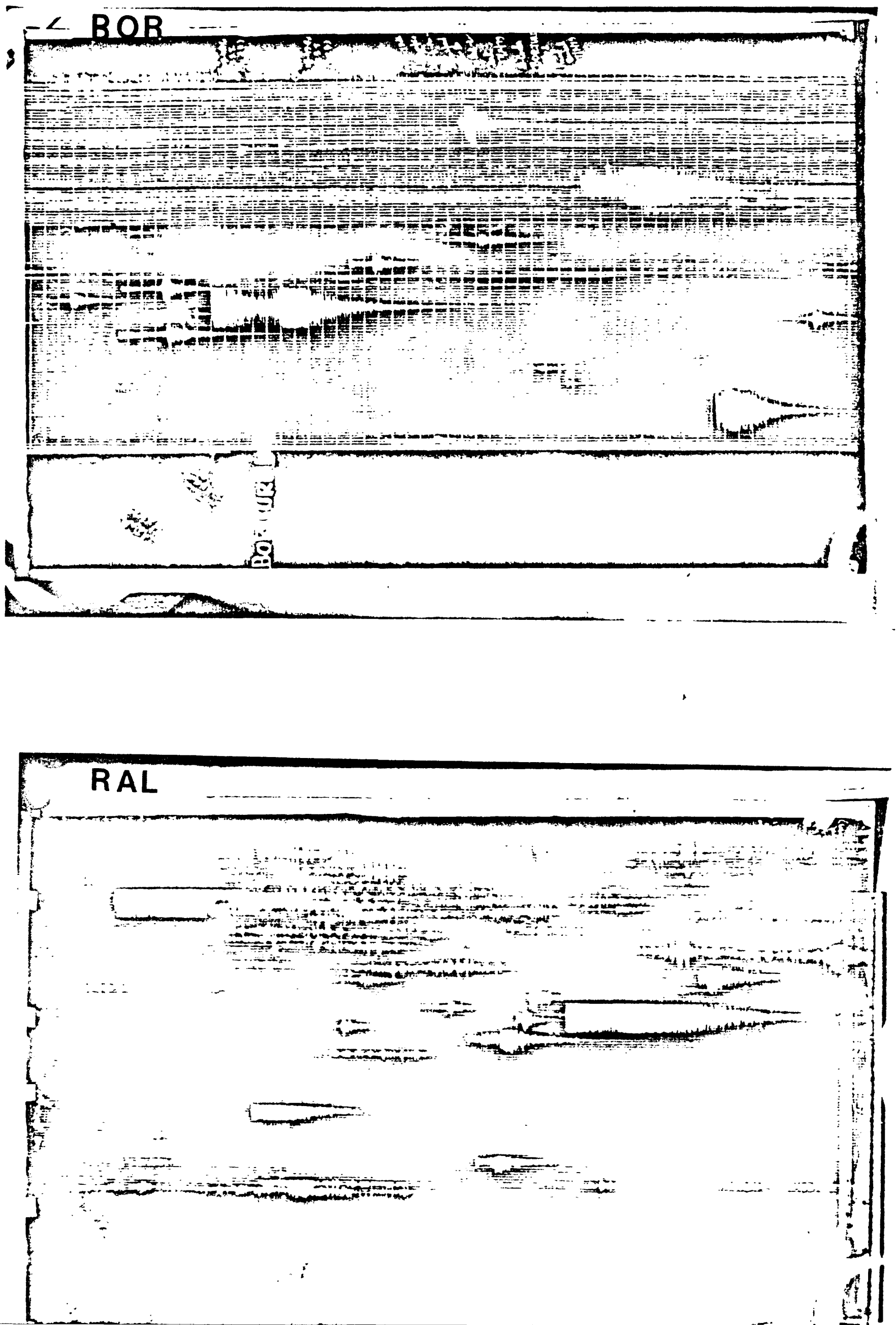


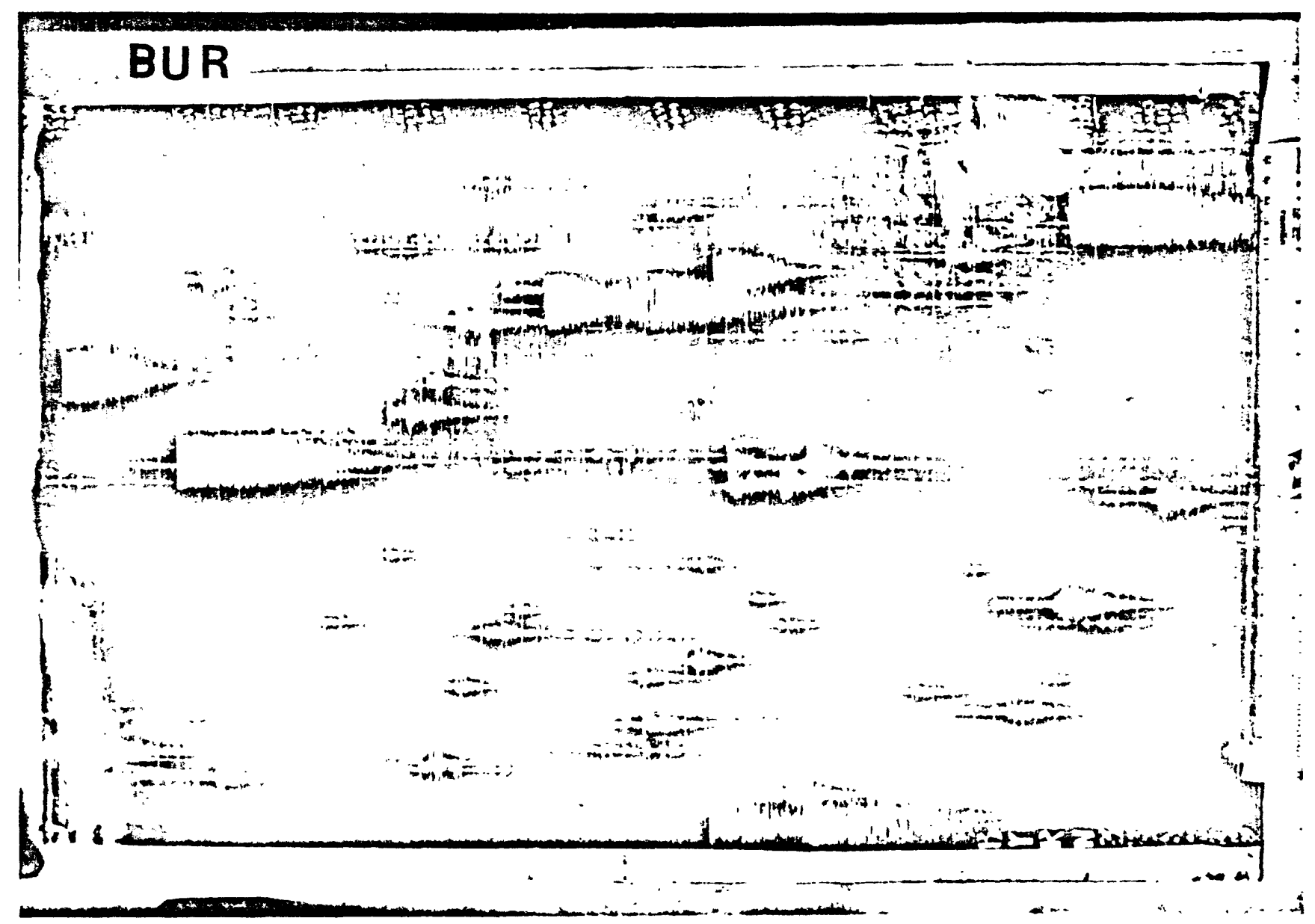




\section{APPENDIX B}

$$
28 \text { pages }
$$

Preliminary Phase Data for Assam 1979

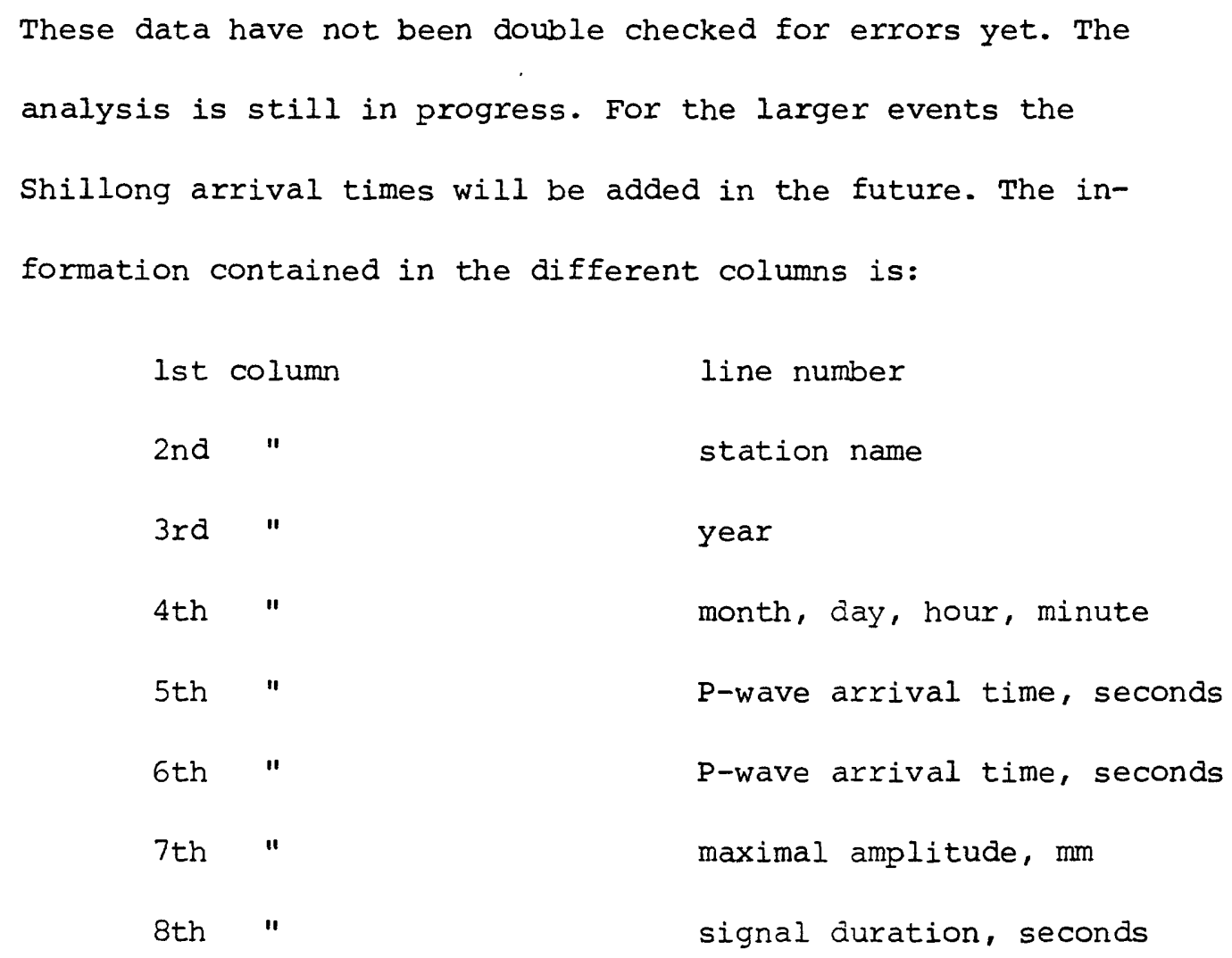




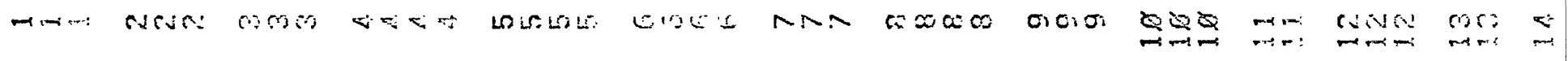

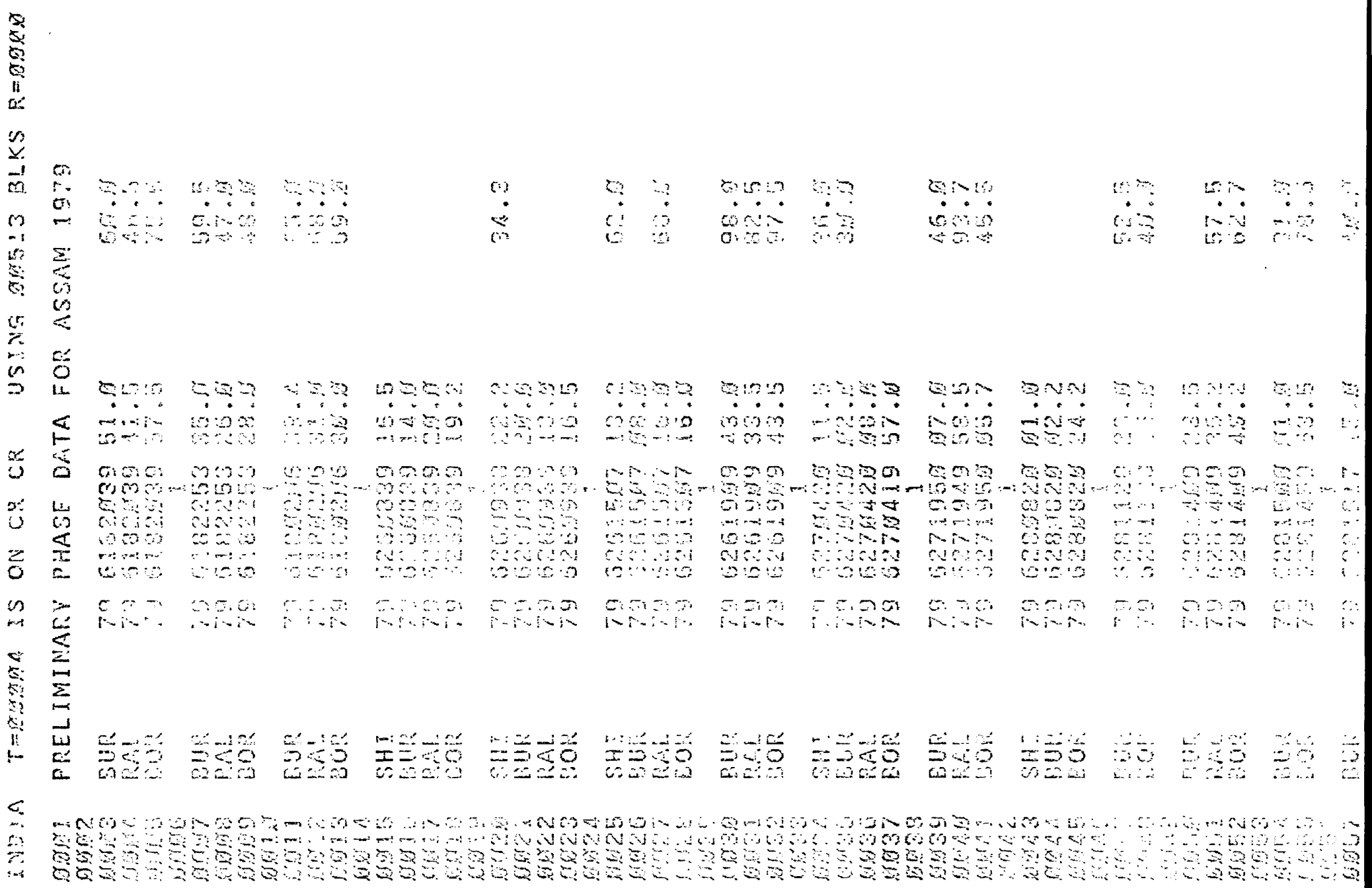




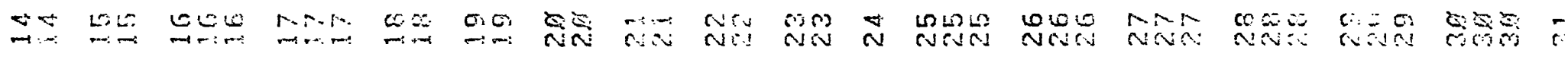

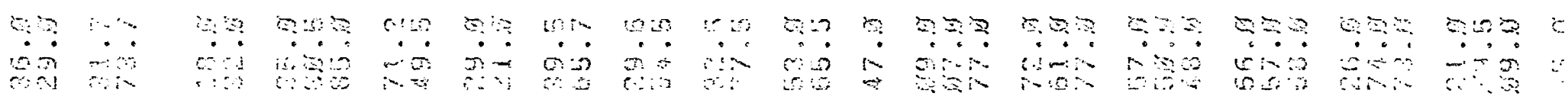

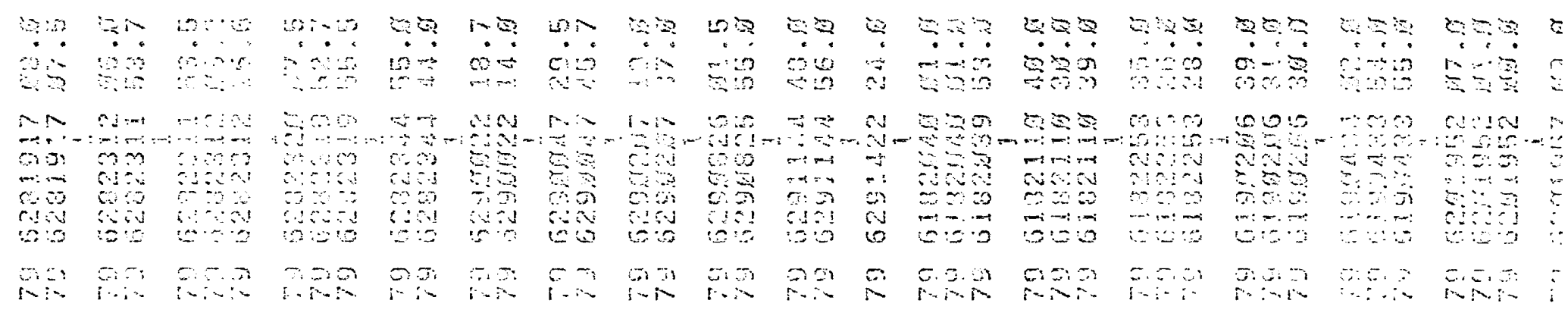

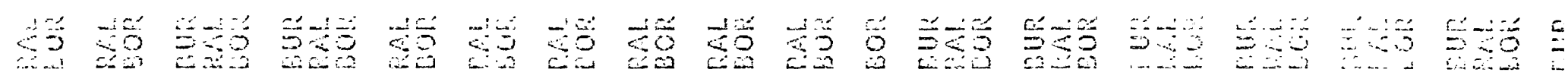
балан E \% 


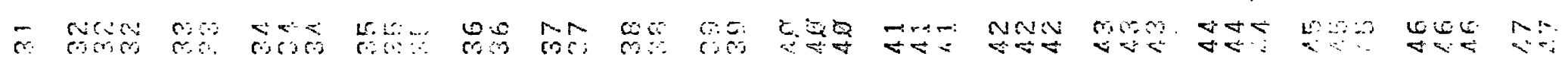

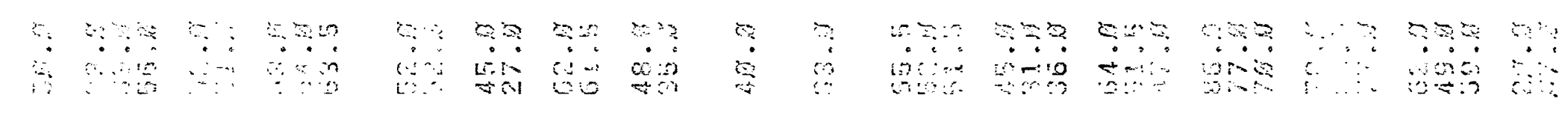

\begin{tabular}{|c|c|c|c|c|c|c|c|c|c|c|c|c|c|c|c|c|}
\hline $\begin{array}{l}3 \\
\vdots \vdots \\
\vdots \vdots\end{array}$ & 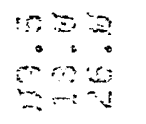 & $\begin{array}{l}E:= \\
\therefore: \\
\therefore: !\end{array}$ & $\begin{array}{r}\because 5: 3 \\
\therefore=3 \\
\because \because 4\end{array}$ & 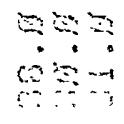 & 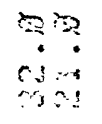 & 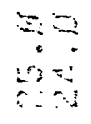 & $\begin{array}{l}5=0 \\
50 \\
0\end{array}$ & $\begin{array}{l}=0 \\
\vdots: 3 \\
\vdots: 3\end{array}$ & 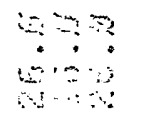 & $\begin{array}{l}0:= \\
6: 3 \\
0: \because\end{array}$ & 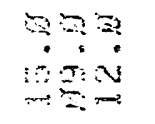 & 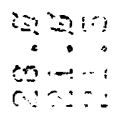 & 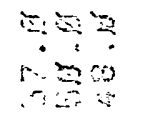 & 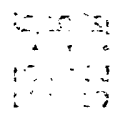 & 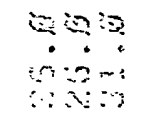 &  \\
\hline , & 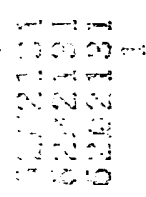 & 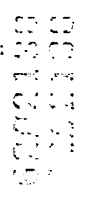 & 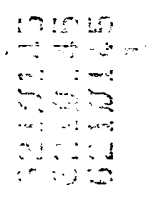 & 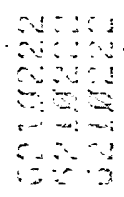 & 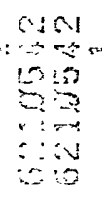 & 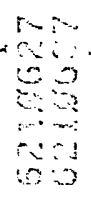 & 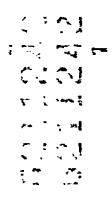 & 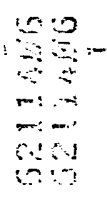 & 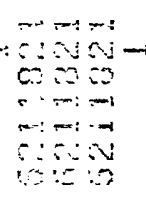 & 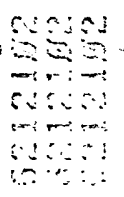 & 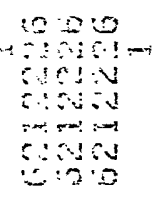 & 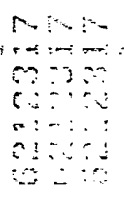 & 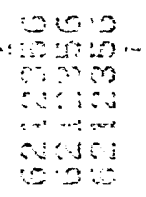 & & 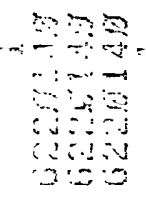 & -3 \\
\hline & $\begin{array}{l}\because \quad \therefore \% \\
\therefore: \because\end{array}$ & $\because \cdots$ & $\begin{array}{l}a r \\
\vdots \cdots\end{array}$ & $\cdots$ & $\because \square$ & 50 & $\because \because$ & $\begin{array}{l}\pi \\
\mathrm{m}\end{array}$ & $\begin{array}{l}\therefore \cdots \infty \\
i \cdots+\infty\end{array}$ & 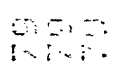 & $\begin{array}{l}n: m=1 \\
i=n\end{array}$ & $\begin{array}{l}\because \\
\therefore i=0\end{array}$ & & $\therefore$ & $\begin{array}{l}m a-1 \\
\therefore i=a\end{array}$ & \\
\hline
\end{tabular}

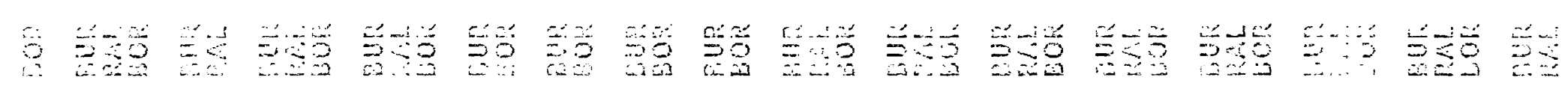

N a-d \% 


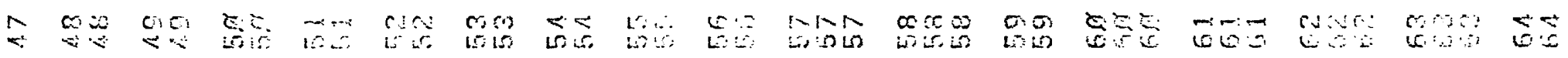

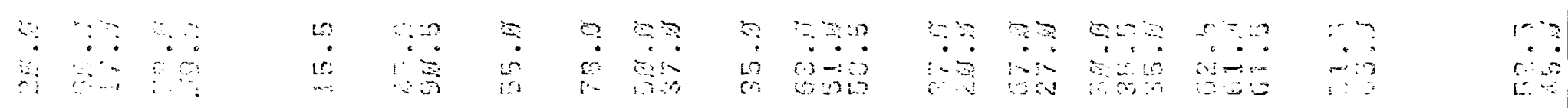

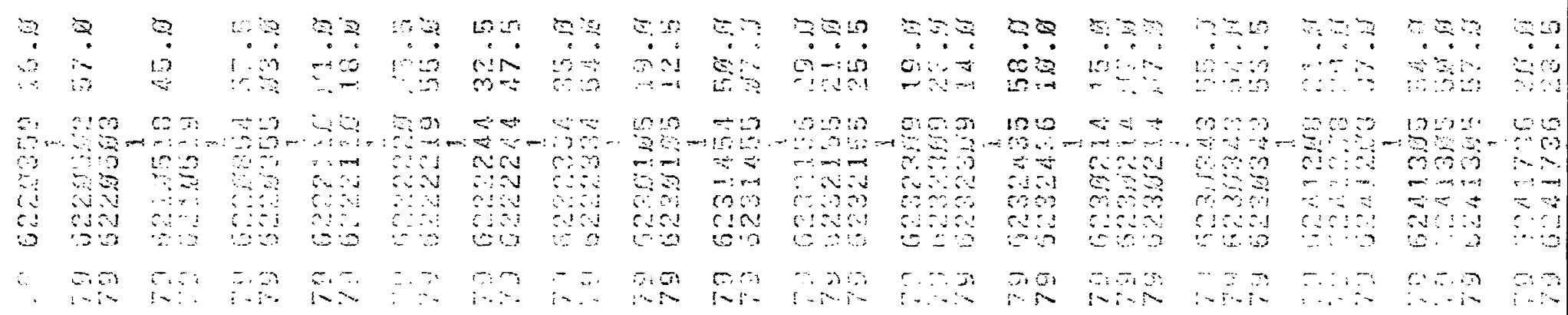

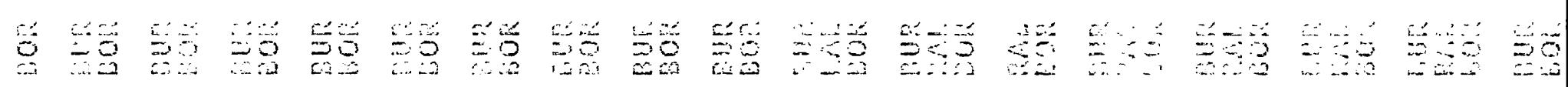

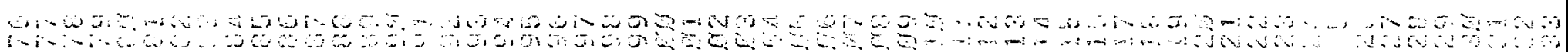
a

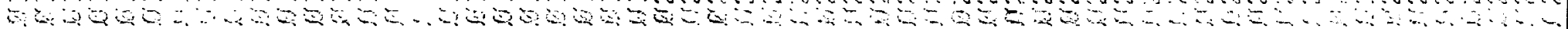




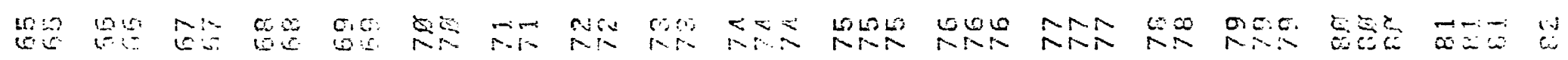

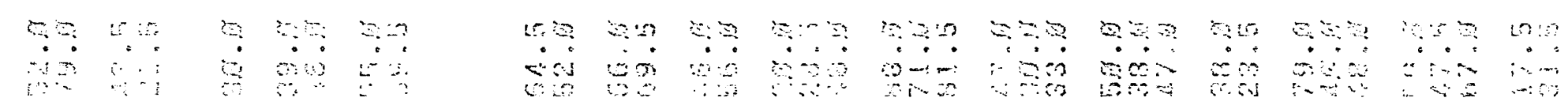

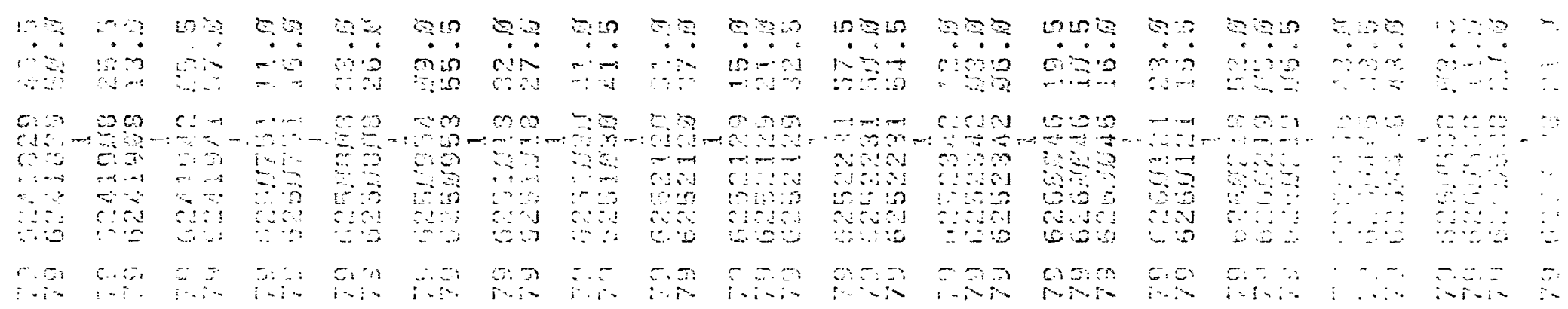

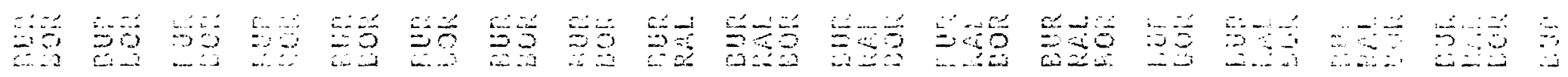

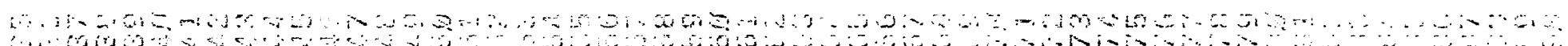

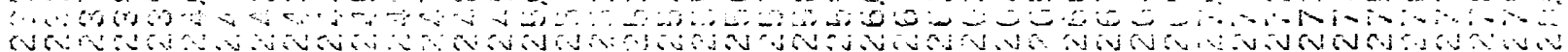
r 


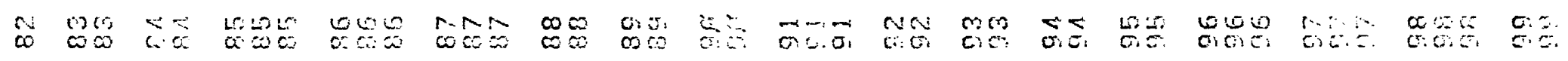

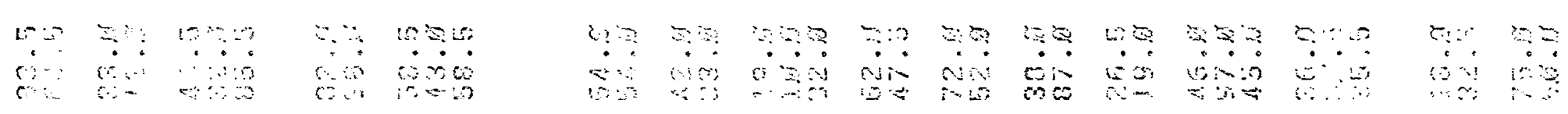

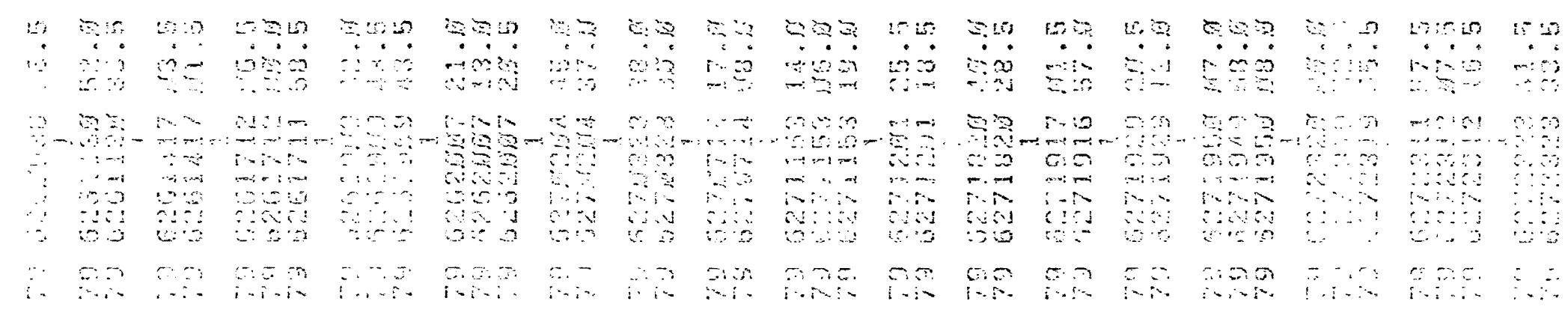

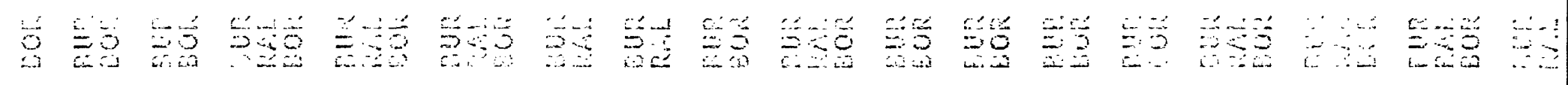

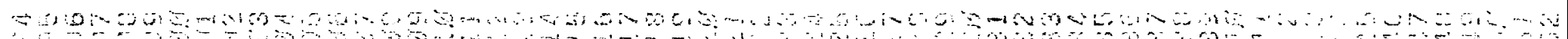

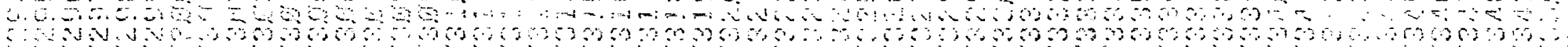

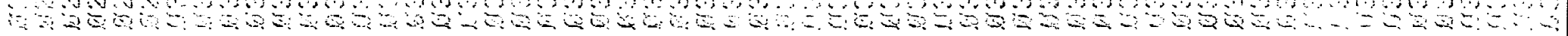




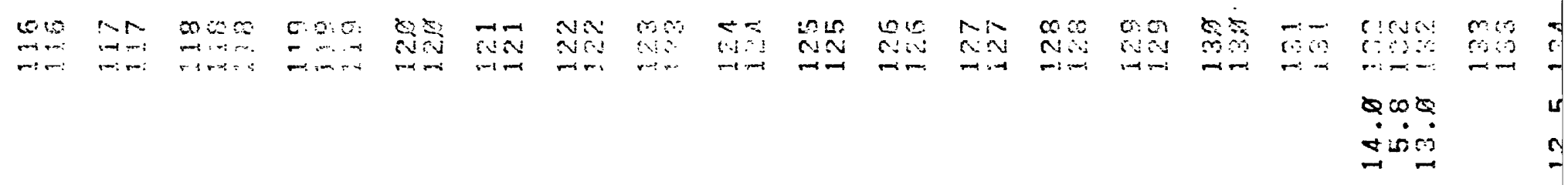

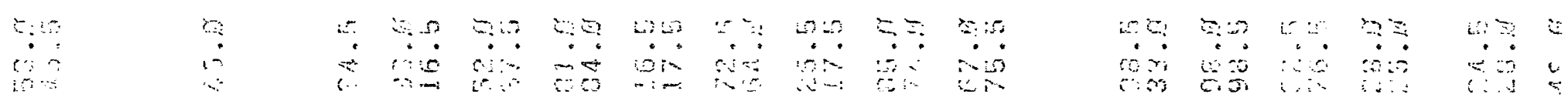

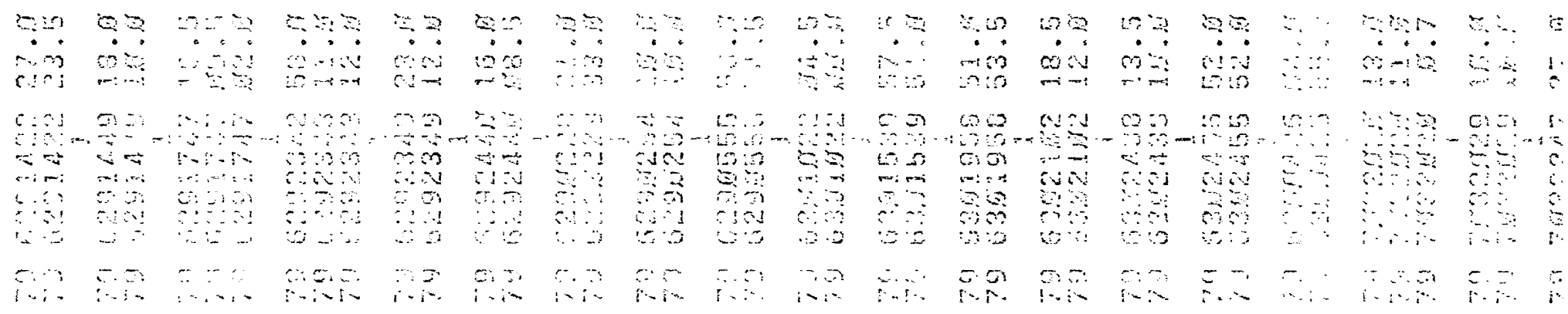

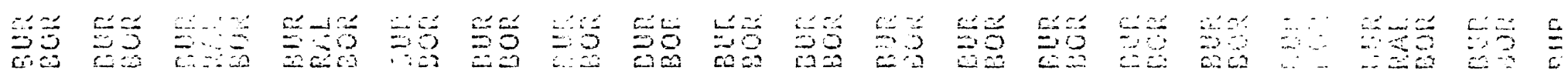

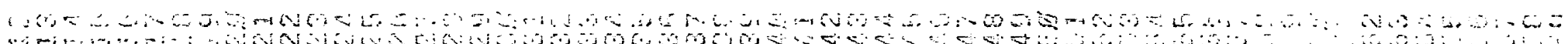

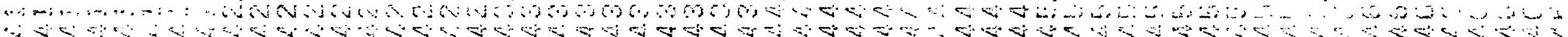
Cat 


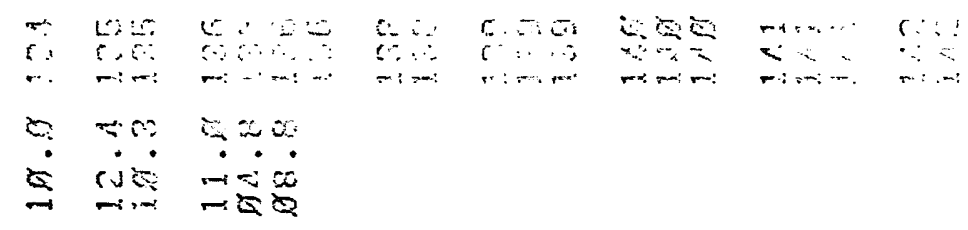

is:

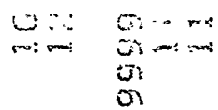

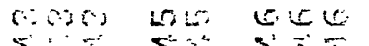

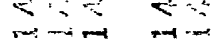
$+\infty$ risio Q cos mirco

बं $\dot{0} \dot{0}$ nin $;$ $\because \div$ 65 is sin $\quad$ in c. .5 $\because \because \cdots$

Li: $\operatorname{Li}_{i \rightarrow \infty} \quad \ldots$

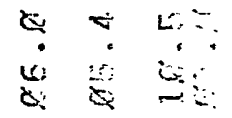

4528 H.

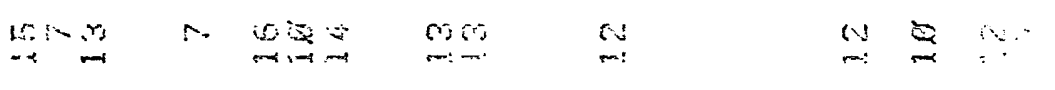

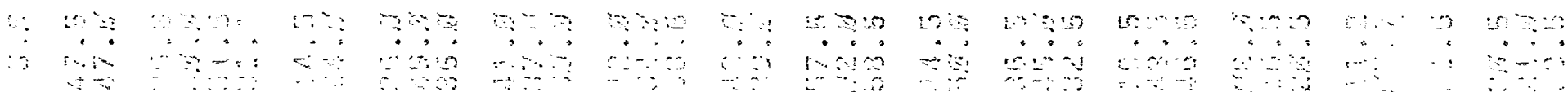

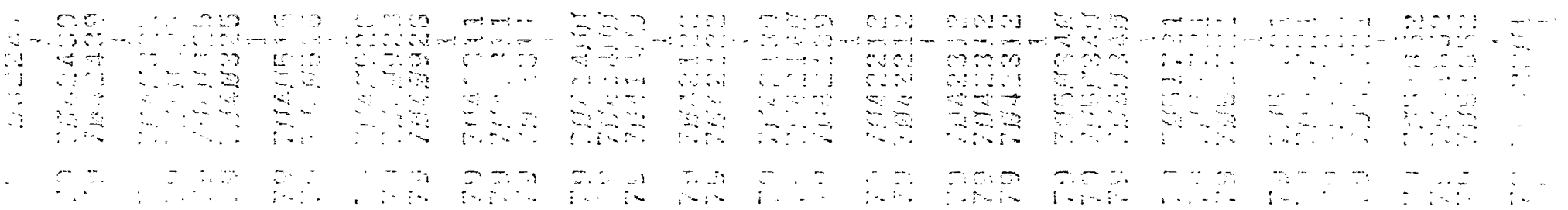

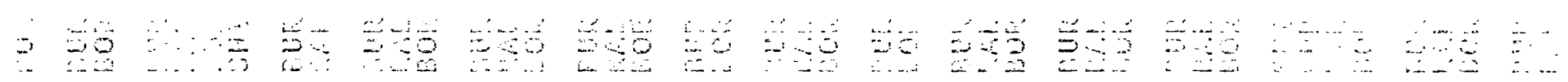

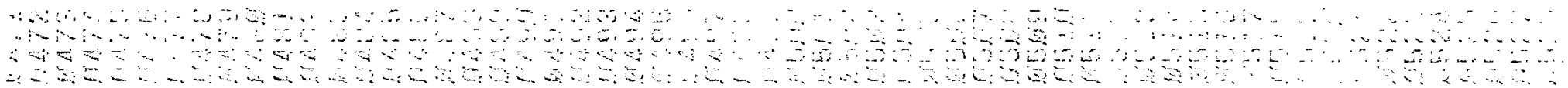




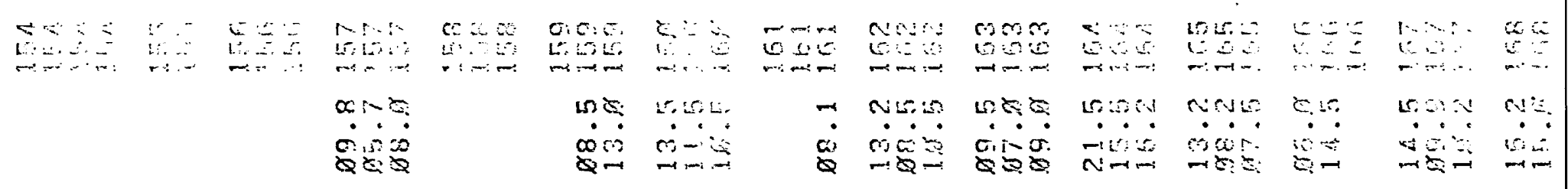

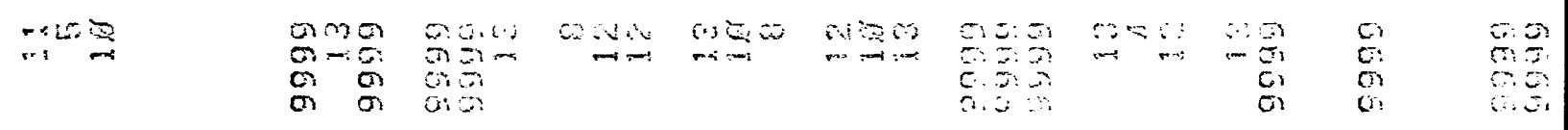

BAm

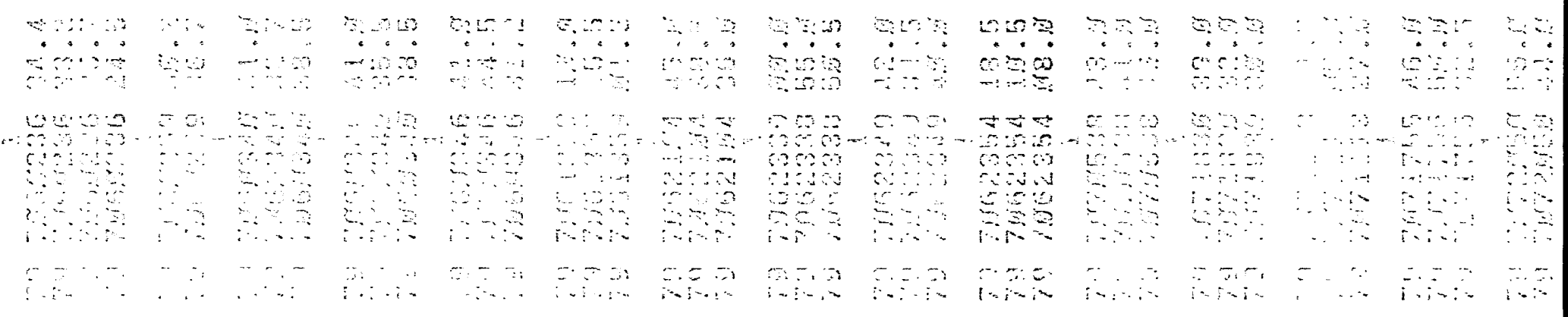

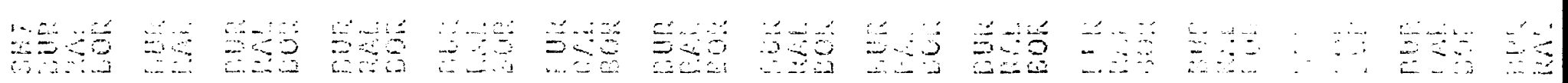
W. 

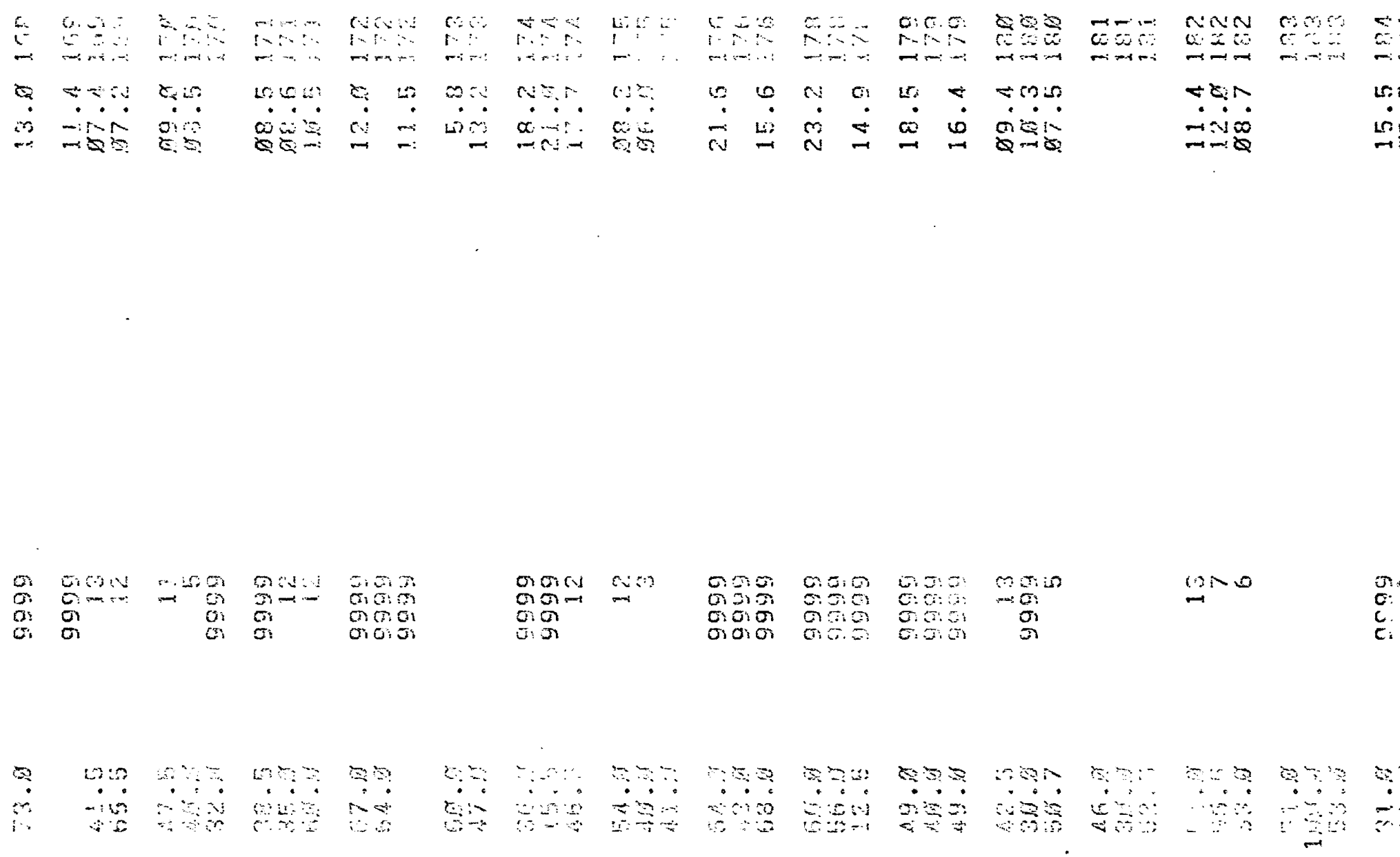

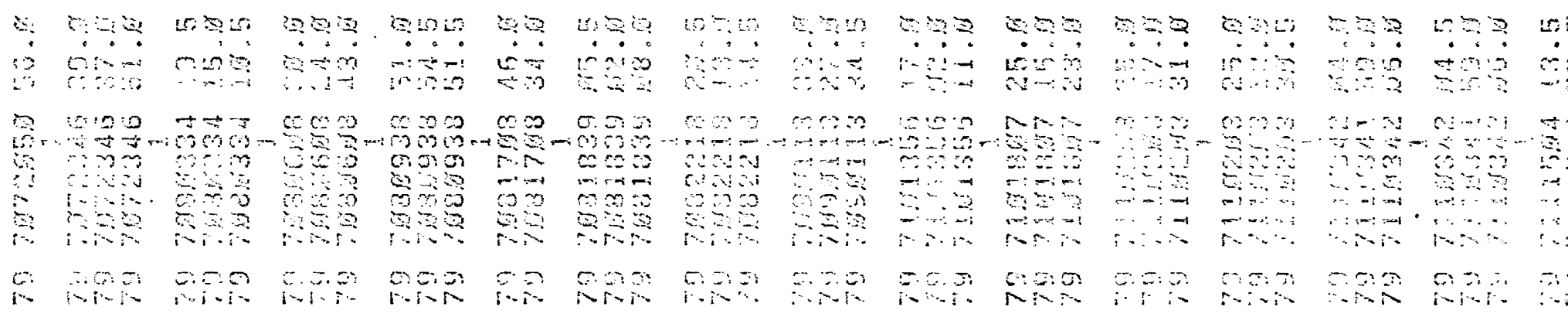

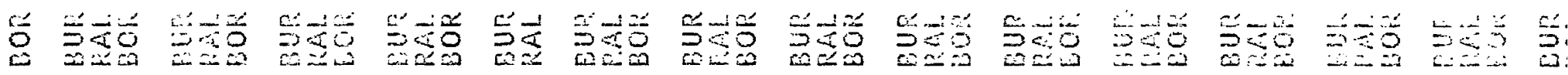

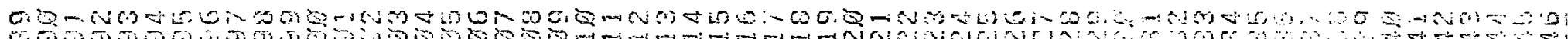

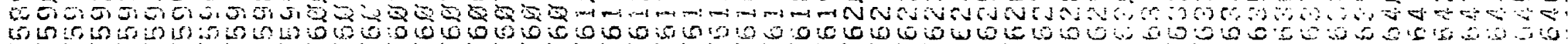
o 


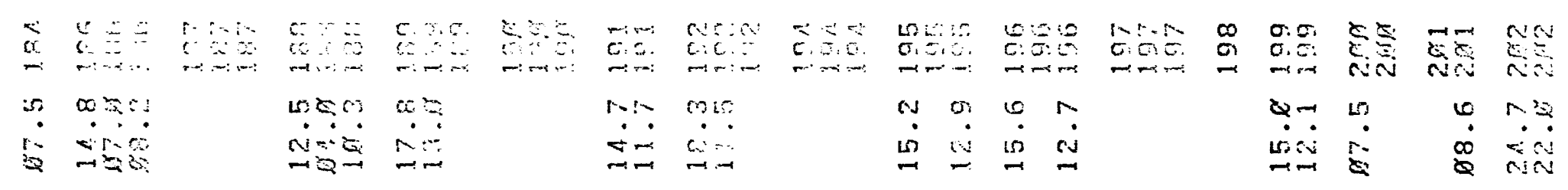

\begin{tabular}{|c|c|c|c|c|c|c|c|c|c|c|}
\hline 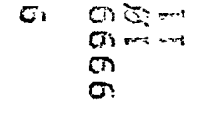 & $\pm=$ & $\begin{array}{l}\text { gan } \\
\text { बian } \\
\text { ang }\end{array}$ & $\begin{array}{c}n g \\
0 \\
0 \\
0\end{array}$ & $\begin{array}{l}\sin \\
6,0 \\
6:\end{array}$ & 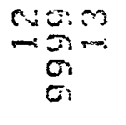 & $\begin{array}{l}-95 \\
050 \\
505\end{array}$ & $\begin{array}{l}\text { ane } \\
\text { angi } \\
0: \sigma i \\
\text { an }\end{array}$ & $N \infty$ & 0 & $\begin{array}{l}5: a \\
5: 0 \\
0: 0\end{array}$ \\
\hline
\end{tabular}

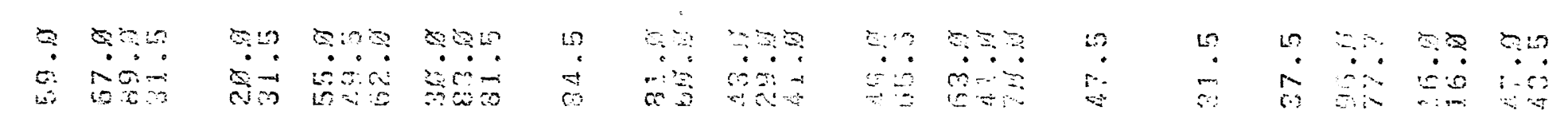

\begin{tabular}{|c|c|c|c|c|c|c|c|c|c|c|c|c|c|c|c|c|}
\hline$\dot{m}$ & 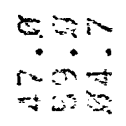 & 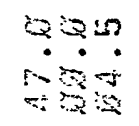 & $\begin{array}{l}500 \\
\therefore m m \\
6 \% 9\end{array}$ & 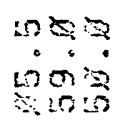 & $\begin{array}{l}10= \\
000 \\
0\end{array}$ & $\begin{array}{l}n \\
0.0\end{array}$ & $\begin{array}{l}53 \\
40 \\
0\end{array}$ & $\begin{array}{l}r 00 \\
\therefore 80 \\
\therefore 3\end{array}$ & $\begin{array}{l}520 \\
800 \\
80\end{array}$ & 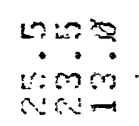 & $\begin{array}{l}50 \\
50 \\
3:-8\end{array}$ & $\stackrel{8}{8}$ & 8 & $\begin{array}{l}30 \\
3\end{array}$ & $\begin{array}{l}8 \\
08 \\
08\end{array}$ & $\begin{array}{l}m \\
\because a \\
\vdots\end{array}$ \\
\hline$\Rightarrow$ & 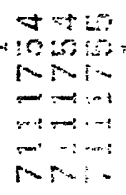 & 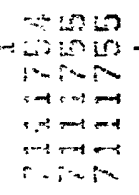 & 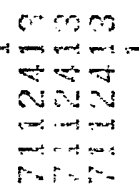 & 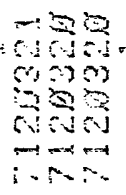 & 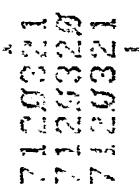 & 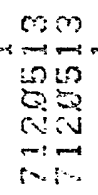 & 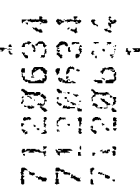 & 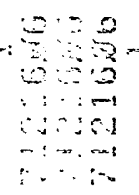 & $\begin{array}{l}0.8 \\
0 \\
0 \\
0\end{array}$ & 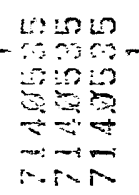 & & $\begin{array}{l}3 \\
m \\
m \\
\cdots \\
\cdots\end{array}$ & 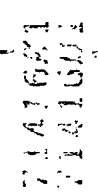 & $=$ & 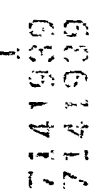 & 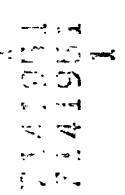 \\
\hline ๓ & $0: 0.03$ & an & 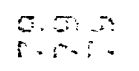 & 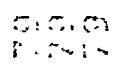 & Den & 90 & $-6-m$ & $\% \nabla$ & 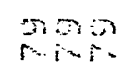 & 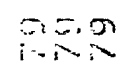 & & 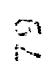 & $\rightarrow$ & & & \\
\hline
\end{tabular}

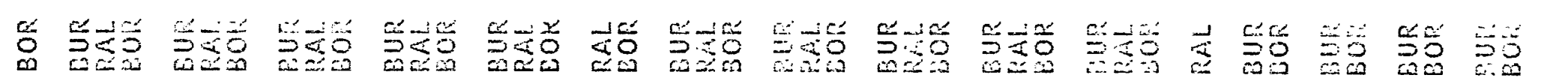

wOF-

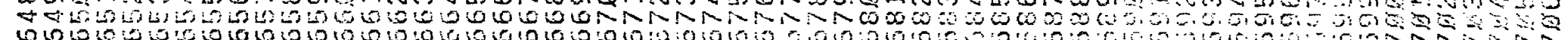

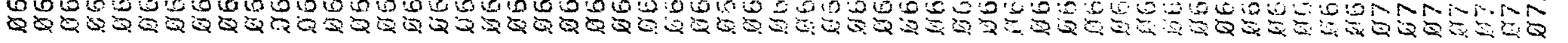




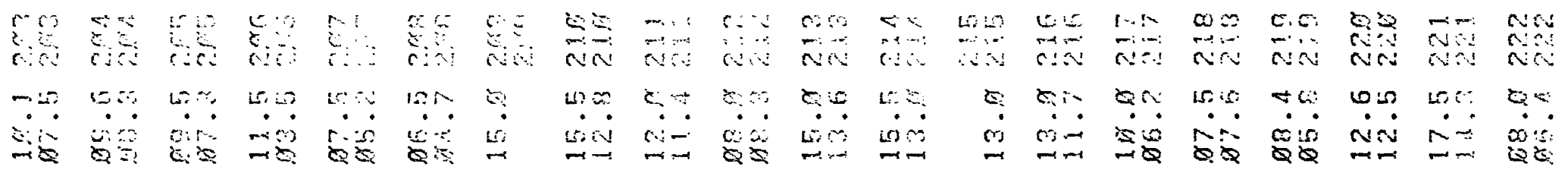

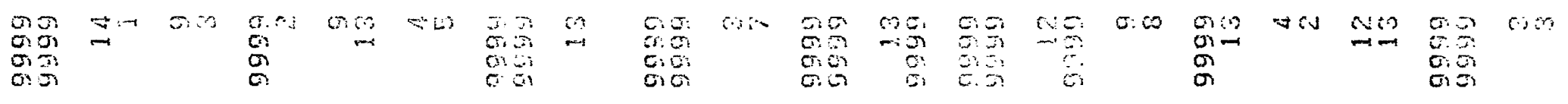

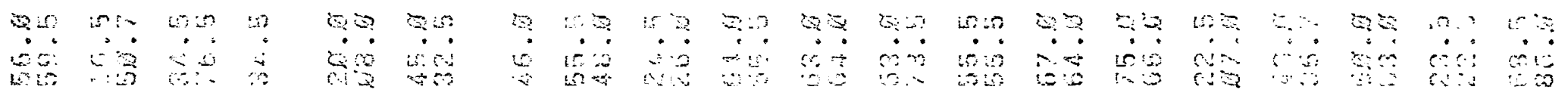

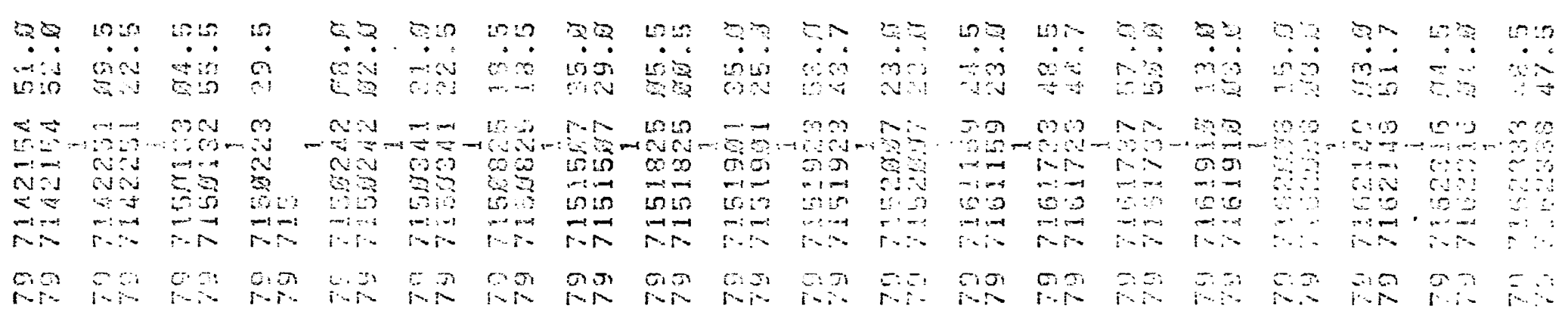

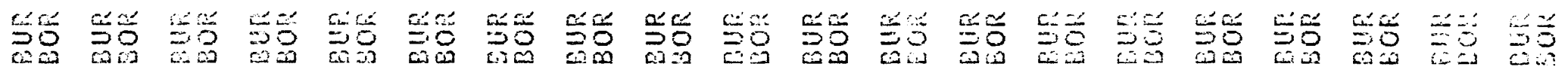

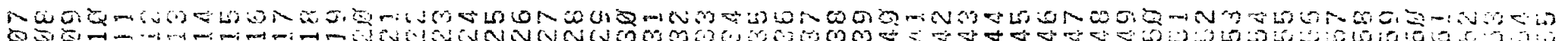

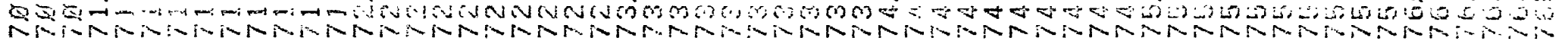

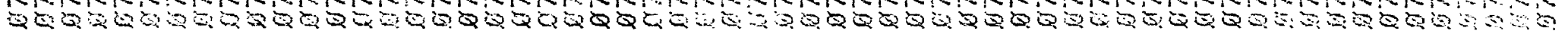




\begin{tabular}{|c|c|c|c|c|c|c|c|c|c|c|c|c|c|c|c|c|}
\hline \multirow{2}{*}{$\begin{array}{l}m s \\
6: \vdots \\
6 \vdots\end{array}$} & 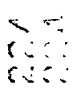 & $\begin{array}{l}\text { Le: } \\
\therefore: 5 \\
\therefore:\end{array}$ & $\begin{array}{l}r \\
\because \\
\therefore\end{array}$ & $\begin{array}{l}1 \cdot 1 \\
6 \because 2 \\
6\end{array}$ & $\begin{array}{l}\because \approx \\
\therefore \therefore \\
\therefore\end{array}$ & $\begin{array}{l}6 \vdots:-5 \\
6 \vdots \vdots \\
6 \vdots\end{array}$ & $\begin{array}{l}25 \\
6 \vdots \\
\therefore\end{array}$ & $\begin{array}{l}-i \\
\therefore \therefore\end{array}$ & $\begin{array}{l}0+1 \\
0: 3\end{array}$ & 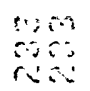 & $\begin{array}{l}5 \\
6 \\
6\end{array}$ & $\begin{array}{l}20 \\
200 \\
0\end{array}$ & $\begin{array}{l}\omega: \frac{1}{6} \\
060\end{array}$ & & $\begin{array}{l}c c i s \\
N \therefore\end{array}$ & \multirow[b]{2}{*}{$\begin{array}{l}250 \\
410 \\
4\end{array}$} \\
\hline & $\begin{array}{l}4 \\
15: \infty \\
-\infty\end{array}$ & $\vec{E} \leqslant$ & $\begin{array}{c}0 \\
0 \\
0\end{array}$ & $\begin{array}{l}1: 2 \\
6 \% \\
80\end{array}$ & $\begin{array}{l}5: 3 \\
-\infty \\
-3\end{array}$ & 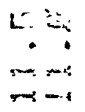 & 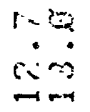 & $\begin{array}{l}\text { L: } \because \\
\therefore \cdots \\
\varepsilon E\end{array}$ & 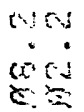 & $\begin{array}{l}15 \approx \\
150 \\
68\end{array}$ & $\begin{array}{c}15:= \\
m= \\
m=\end{array}$ & 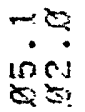 & $\begin{array}{l}4 \\
0\end{array}$ & & 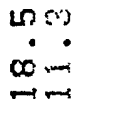 & \\
\hline
\end{tabular}

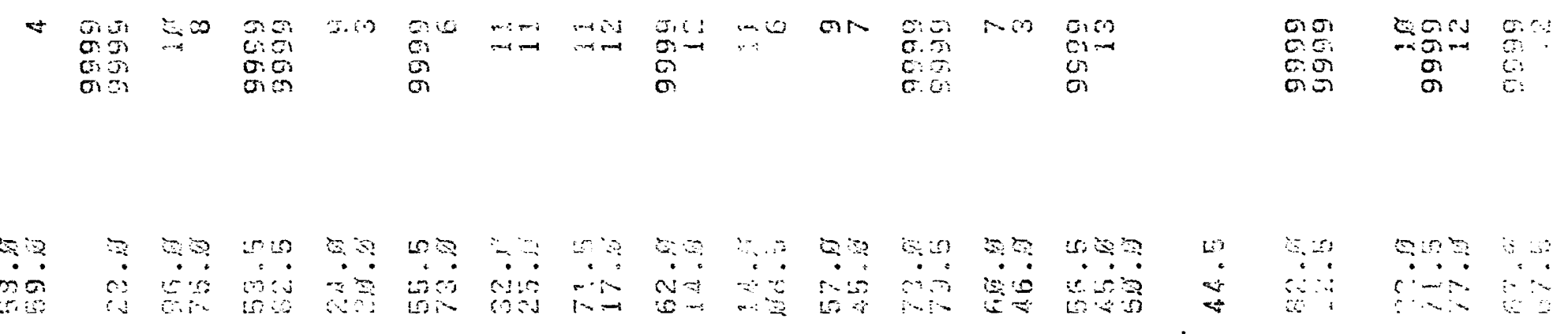

\begin{tabular}{|c|c|c|c|c|c|c|c|c|c|c|c|c|c|c|c|c|c|}
\hline $\begin{array}{l}\text { mis } \\
\text { mis } \\
m\end{array}$ & 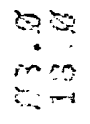 & 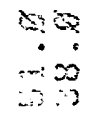 & $\begin{array}{r}4: 3 \\
03 \\
4: 5\end{array}$ & $\begin{array}{l}29 \\
0=9 \\
69\end{array}$ & 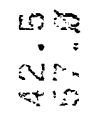 & $\begin{array}{l}\because 3 \\
\therefore 3 \\
\therefore 3\end{array}$ & 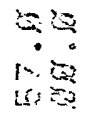 & $\begin{array}{l}6: 4 \\
03 \\
8\end{array}$ & $\begin{array}{l}2= \\
\therefore= \\
\therefore:\end{array}$ & $\begin{array}{l}\infty \vdots \\
\therefore 0 \\
\vdots \infty\end{array}$ & $\begin{array}{l}=: 3 \\
: 5 \\
: ;\end{array}$ & 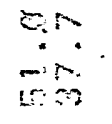 & 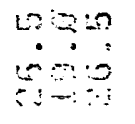 & 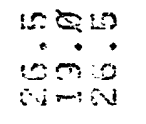 & 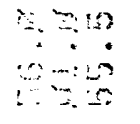 & $\begin{array}{l}6: 5 \\
505 \\
505\end{array}$ & $\begin{array}{l}\because: 3 \\
\because \vdots: \\
\because:\end{array}$ \\
\hline 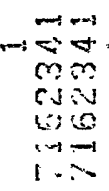 & 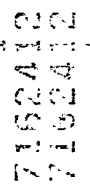 & 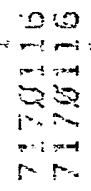 & 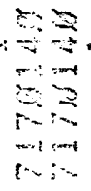 & 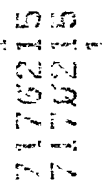 & 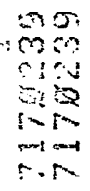 & 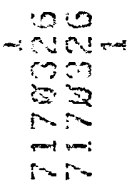 & $\begin{array}{l}\infty \pi \\
\infty m \\
m \infty \\
\infty \\
\infty=\infty \\
1 \rightarrow \infty\end{array}$ & 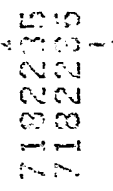 & 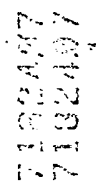 & 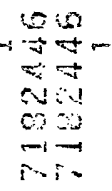 & 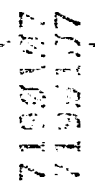 & 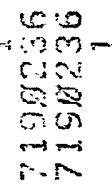 & 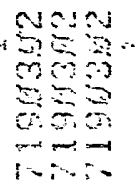 & 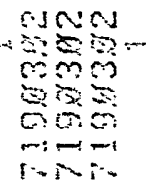 & $\begin{array}{l}\because \\
\because \\
\vdots \\
\vdots\end{array}$ & 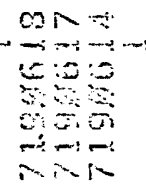 & \\
\hline$S_{i}$ & $\begin{array}{l}5: \cdots \\
5=\vdots\end{array}$ & $\begin{array}{l}2:-7 \\
12\end{array}$ & $\begin{array}{l}c: D \\
\cdots: D\end{array}$ & $5 \pi$ & $\begin{array}{l}3 \pi \\
\forall i=\end{array}$ & & $\begin{array}{l}\sigma i n \\
N R\end{array}$ & 1 & $\begin{array}{l}c= \\
r+i\end{array}$ & $\pi$ & $\because$ & $\pi n$ & & $i$ & & & \\
\hline
\end{tabular}

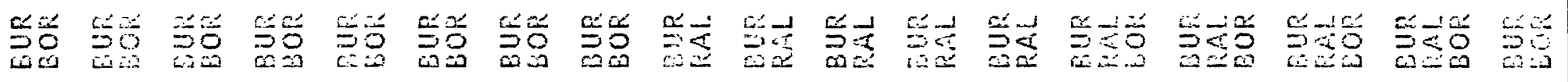

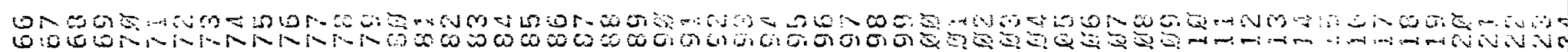

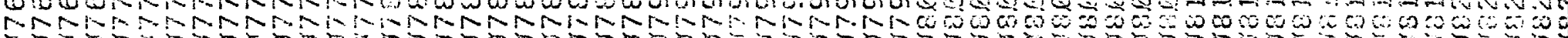
Q 


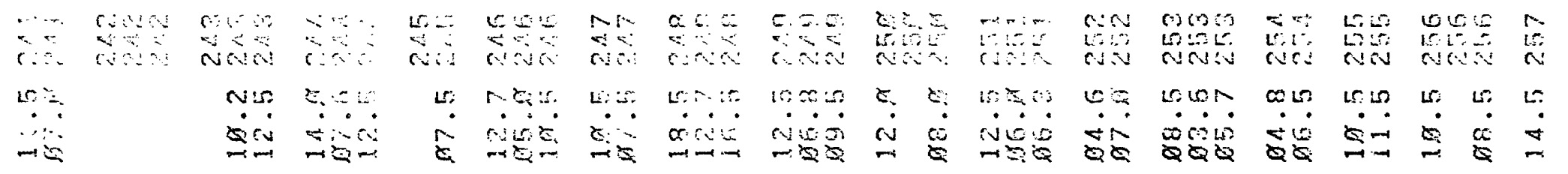

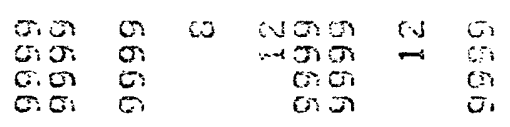

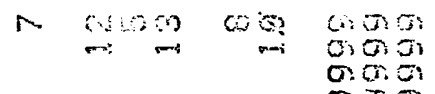
窝我

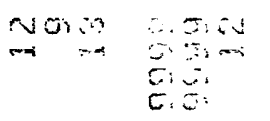
$\div$
ons an 0.5
$\begin{array}{cc}\pi+\pi & 0 \\ 0: & 0 \\ 0: & 5 \\ 61 & 5\end{array}$

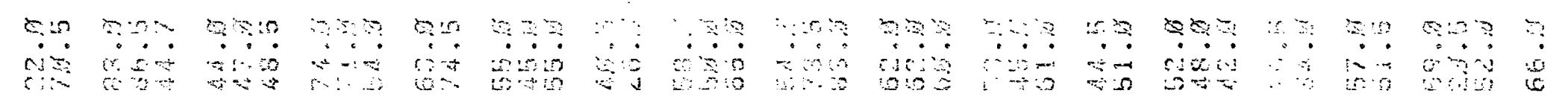

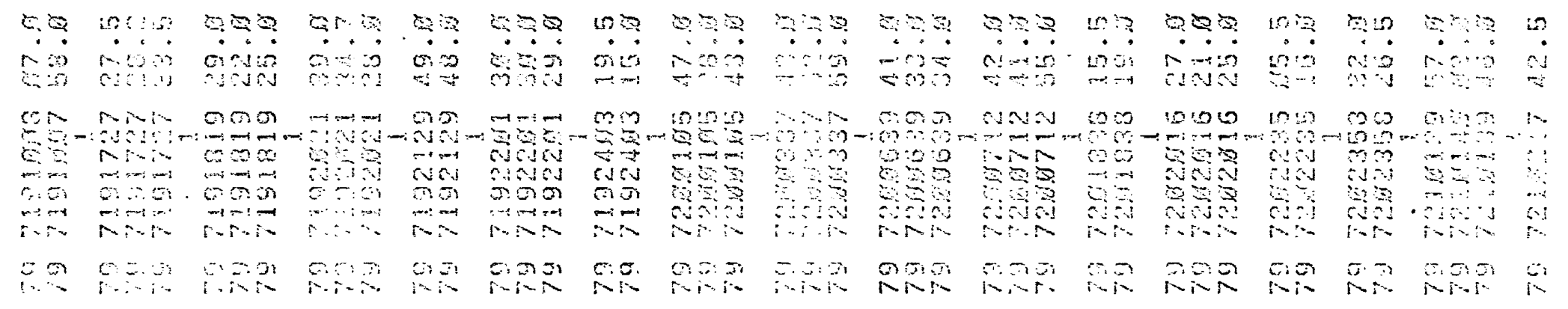

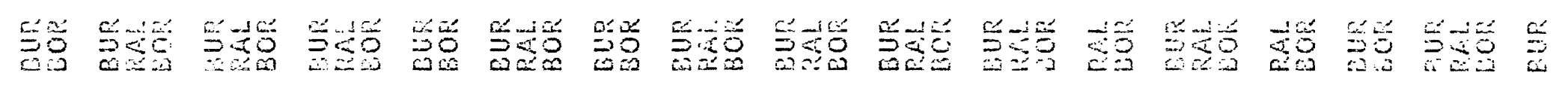

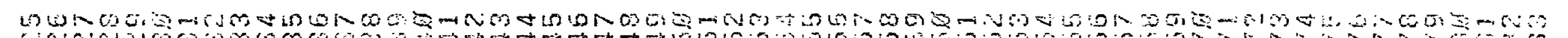

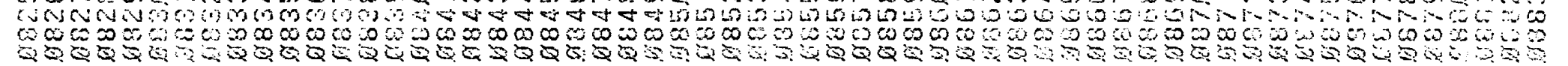




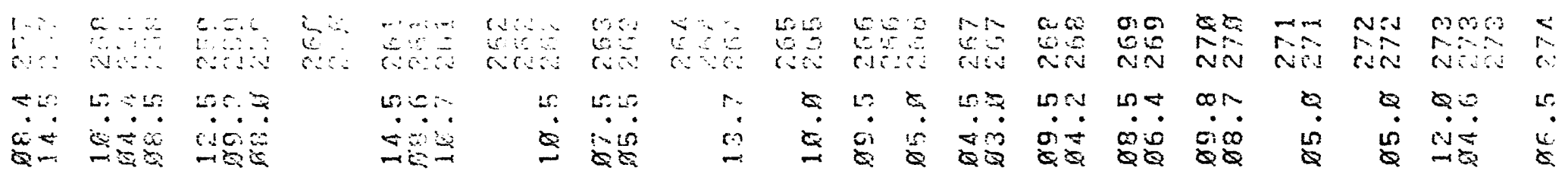

\begin{tabular}{|c|c|c|c|c|c|c|c|c|c|c|c|c|c|c|c|c|c|}
\hline$<\omega$ & 900 & $\cos$ & (1) & $\begin{array}{l}0 \cdot 3 \\
\sigma:-a \\
\sigma: a \\
\sigma \\
\sigma\end{array}$ & 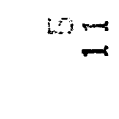 & 60 & $\begin{array}{ll}5 & 0 \\
0 & 0 \\
G & 0 \\
\sigma & 0\end{array}$ & 120 & $\Rightarrow m$ & $\therefore=$ & Eus & 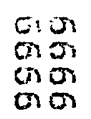 & $\begin{array}{l}0: 0 \\
5: 5 \\
6: 5 \\
\sigma:\end{array}$ & $m$ & $\begin{array}{c}M a \\
G \\
\text { a } \\
\text { on }\end{array}$ & $\begin{array}{c}\omega \sigma \\
\sigma \\
\sigma \\
\sigma\end{array}$ & $=$ \\
\hline $5:$ & 0 & $8 \%$ & $\because$ & 89 & $n$ & 20 & $\because>3$ & $\because \because$ & $\because a n$ & $=$ & min & ? & $\therefore: ?$ & & 5 & $3: 0$ & \\
\hline$\therefore$ & $46-2$ & 90 & $\begin{array}{c}40 \\
x\end{array}$ & $\begin{array}{l}\text { Was } \\
\text { nof }\end{array}$ & $-3-5$ & $\therefore$ & $\because \vdots$ & $\therefore$ & 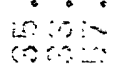 & $\therefore \dot{*}$ & 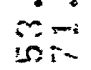 & $\therefore i$ & -8 & 8 & $8 \dot{s}$ & ro & 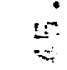 \\
\hline
\end{tabular}

\begin{tabular}{|c|c|c|c|c|c|c|c|c|c|c|c|c|c|c|c|c|}
\hline . & 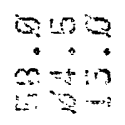 & $\begin{array}{l}9 s: ? \\
n=6\end{array}$ & $\begin{array}{l}90 \\
90 \\
90\end{array}$ & 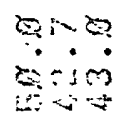 & 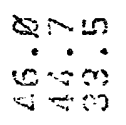 & $\begin{array}{l}015 \\
85 \\
0\end{array}$ & 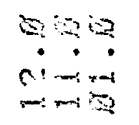 & 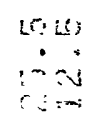 & 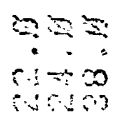 & $\begin{array}{l}39 \\
\because 3 \\
30\end{array}$ & $\begin{array}{l}3 \\
0 \\
0 \\
0\end{array}$ & $\begin{array}{l}20 \\
6 \infty \\
60\end{array}$ & $\begin{array}{l}\therefore: 5 \\
\therefore: 3\end{array}$ & 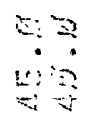 & $\begin{array}{l}\because ? \\
\therefore \infty \\
6\end{array}$ & \begin{tabular}{l}
$3: ?$ \\
$0: 0$ \\
\hdashline \\
\hdashline
\end{tabular} \\
\hline 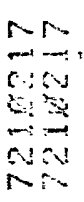 & 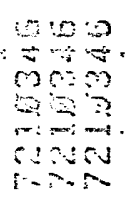 & 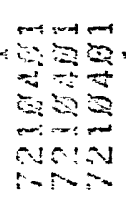 & 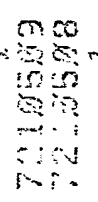 & 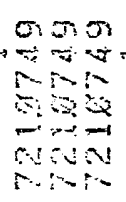 & 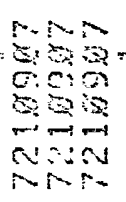 & 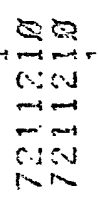 & 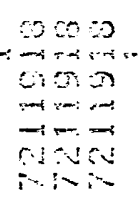 & 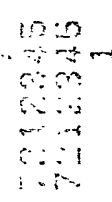 & 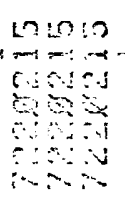 & 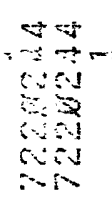 & $\begin{array}{c}0 \\
610 \\
610\end{array}$ & 8 & $\theta$ & & & 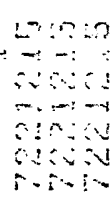 \\
\hline & & & & 0 & 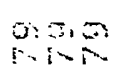 & $\Xi$ & & & 50 & & & & & & & \\
\hline
\end{tabular}

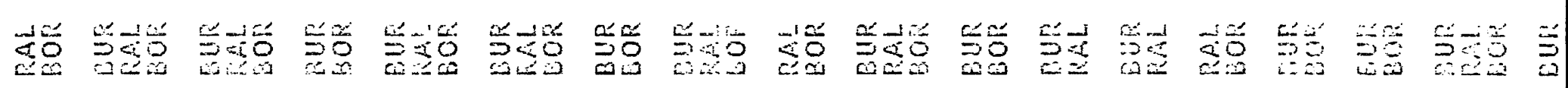

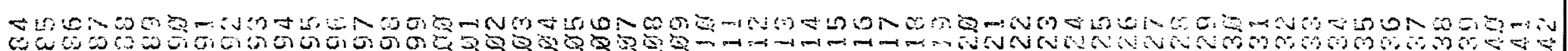

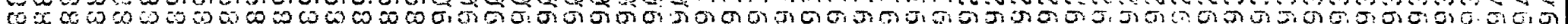

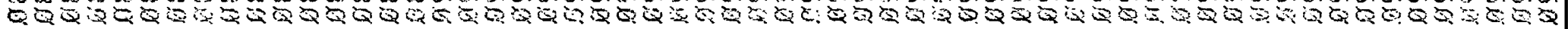




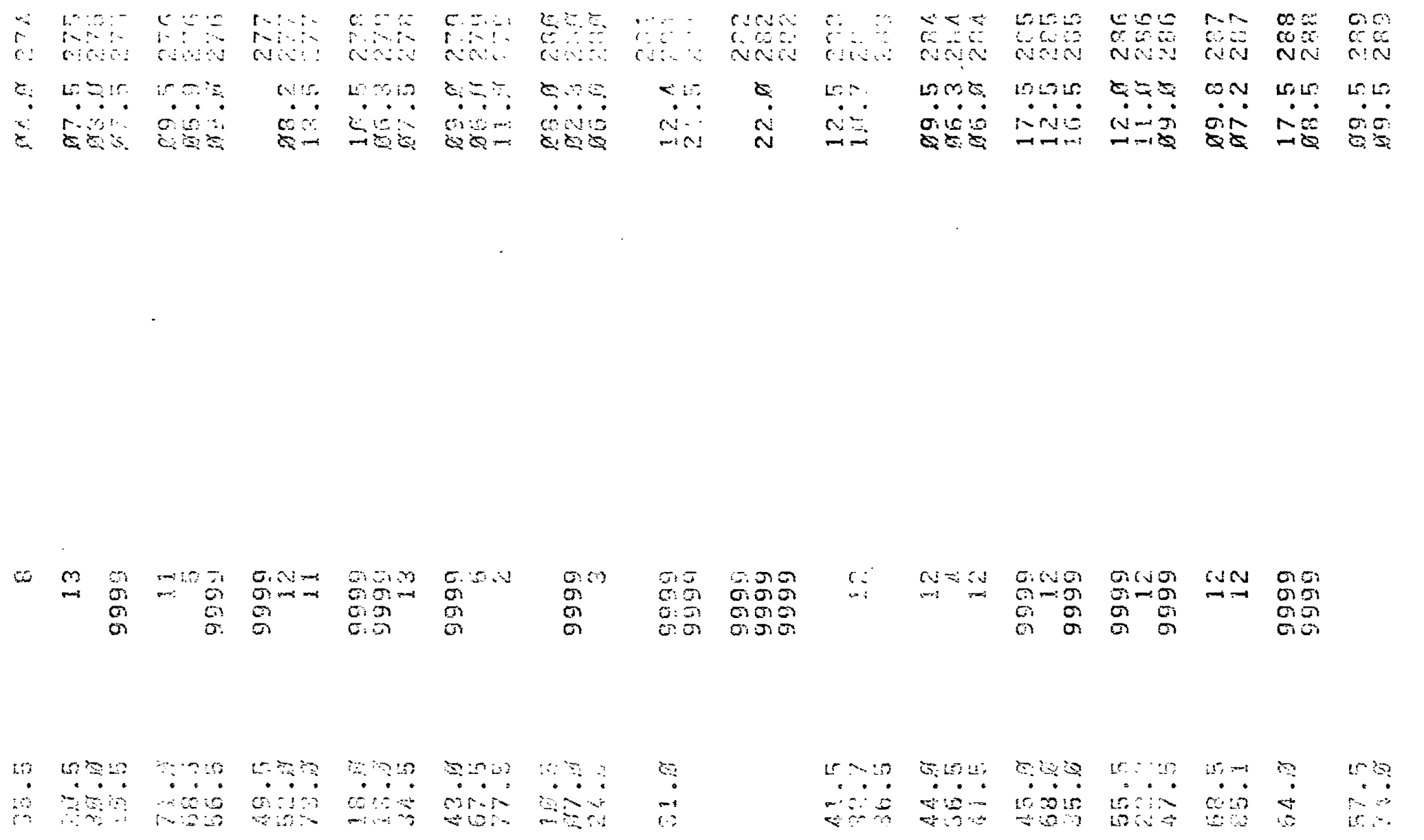

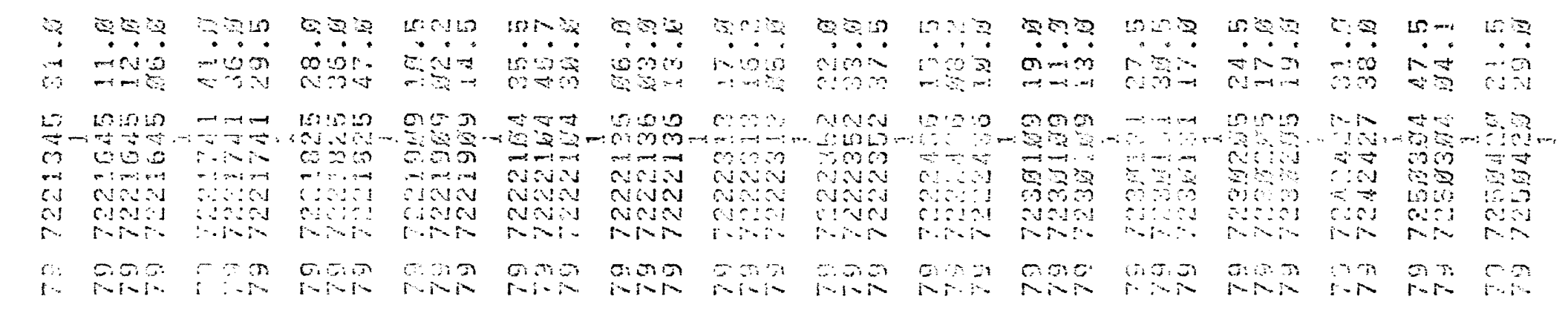

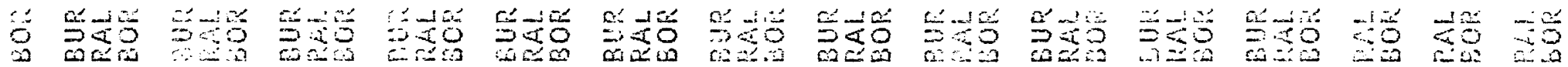

M.7.

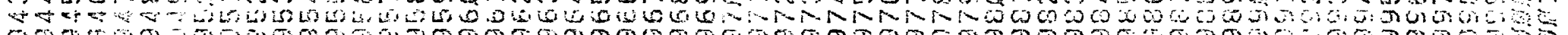

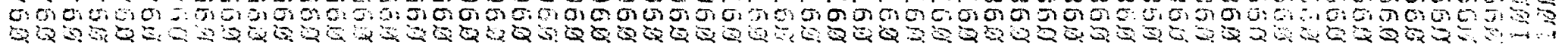




\begin{tabular}{|c|c|c|c|c|c|c|c|c|c|c|c|c|c|c|c|c|}
\hline $\begin{array}{l}5 \\
2 \\
2\end{array}$ & 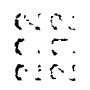 & $\begin{array}{l}\because \therefore \\
0 \therefore: \\
\therefore \therefore\end{array}$ & $\begin{array}{l}3 \\
2 \\
2\end{array}$ & 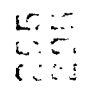 & 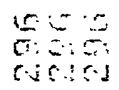 & 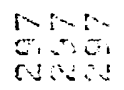 & $\begin{array}{lll}0 & 0 \\
0 & 0 \\
0 & 0\end{array}$ & $\begin{array}{l}c: \bar{\sigma} \\
\sigma \\
\therefore\end{array}$ & cis & $\begin{array}{l}-5= \\
650\end{array}$ & $\begin{array}{l}000 \\
6000 \\
600\end{array}$ & $\begin{array}{l}m \infty n \\
n 20\end{array}$ & 60 & $\frac{\cos }{\cos }=\frac{2}{6}$ & $\begin{array}{l}\omega s \\
\mathrm{~s} \\
\mathrm{~m}\end{array}$ & 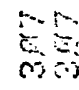 \\
\hline 5 & $\stackrel{n}{a}$ & $\begin{array}{l}r i s \\
m=\end{array}$ & $\therefore$ & $\dot{\square}$ & $\begin{array}{l}Q: 5 \\
5: 5 \\
-\cdots\end{array}$ & $\begin{array}{l}n: 5: \\
\dot{s i s}\end{array}$ & 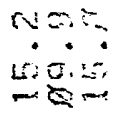 & $\underset{m}{0+\infty}$ & $\begin{array}{l}r i s \\
0: 3 \\
0 \%\end{array}$ & $\begin{array}{l}N \ll< \\
t:+2 \\
m-i=1\end{array}$ & $\begin{array}{l}\text { bis: } 0 \\
0 \div 0 \\
0=0\end{array}$ & & $\begin{array}{l}5: 5 \\
\vdots: \\
\omega: 5\end{array}$ & $\begin{array}{l}+5 \\
m=\dot{3} \\
=8\end{array}$ & $\begin{array}{l}N:=? \\
0: 5=0 \\
N=-4\end{array}$ & تص \\
\hline
\end{tabular}

\begin{tabular}{|c|c|c|c|c|c|c|c|c|c|c|c|c|c|c|c|c|c|c|}
\hline 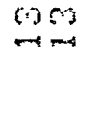 & $\begin{array}{l}0: 0 \\
010 \\
0.0 \\
010\end{array}$ & $\begin{array}{l}a n \\
0.0 \\
0.0\end{array}$ & 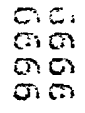 & $\begin{array}{l}6 \\
6 \\
6\end{array}$ & 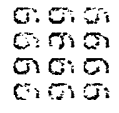 & $\begin{array}{c}m, a \\
0 \\
0 \\
0\end{array}$ & 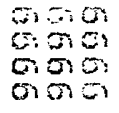 & 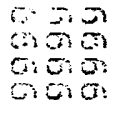 & 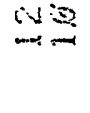 & 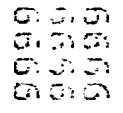 & $\begin{array}{l}0 \\
6 \\
6\end{array}$ & $\begin{array}{l}5 \\
\tilde{\sigma} \\
0\end{array}$ & $\begin{array}{l}\infty \pi \\
0 \Omega \\
0 n \\
0 \pi\end{array}$ & $\begin{array}{l}9 \\
0 \\
0\end{array}$ & $\begin{array}{l}0 i a \\
\text { ain } \\
0 \\
0\end{array}$ & $\begin{array}{l}c: \tau: \\
\tilde{E}: \\
\pi\end{array}$ & $\begin{array}{l}5 \\
5 \\
0 \\
0\end{array}$ & 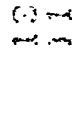 \\
\hline
\end{tabular}

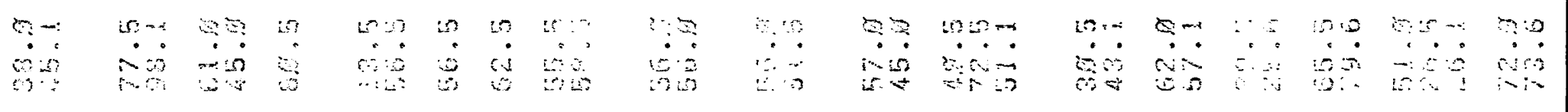

\begin{tabular}{|c|c|c|c|c|c|c|c|c|c|c|c|c|c|c|c|c|}
\hline $\begin{array}{l}?=2 \\
\dot{0}=2\end{array}$ & $\begin{array}{l}3+\infty \\
+\infty\end{array}$ & 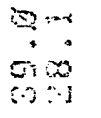 & 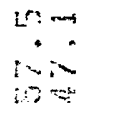 & 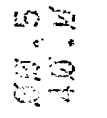 & $\underset{m i n}{40}$ & $\begin{array}{l}\dot{m}: 0 \\
\dot{0}=0\end{array}$ & 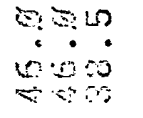 & 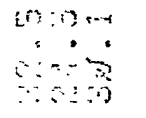 & $\begin{array}{l}-3: 0 \\
: 10 \\
\because 0\end{array}$ & 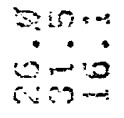 & 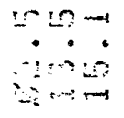 & 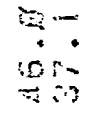 & $\begin{array}{l}0 \% \\
\therefore \therefore \\
\therefore \cdots\end{array}$ & $\begin{array}{l}10-5 \\
10 \cdot 0 \\
10\end{array}$ & 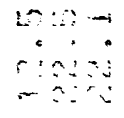 & 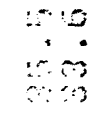 \\
\hline $\begin{array}{l}53 \\
602 \\
63 \\
50 \\
20\end{array}$ & 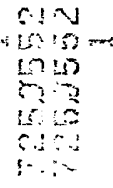 & 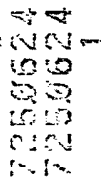 & 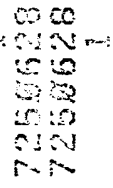 & 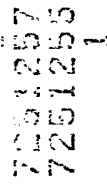 & 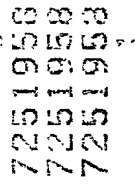 & 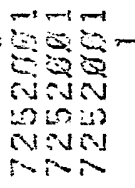 & 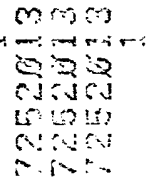 & 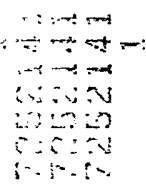 & 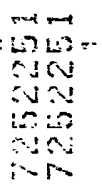 & 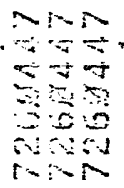 & 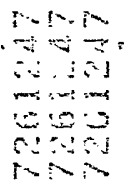 & 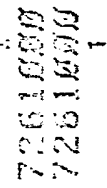 & 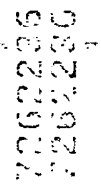 & 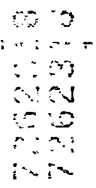 & $\because 301$ & \\
\hline$\therefore$ & $m i$ & $=\bar{i}$ & $G \theta$ & $0 \%$ & :90 & Mn & 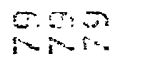 & 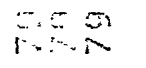 & 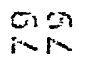 & $a \cdots$ & $\begin{array}{l}s=0 \\
r i n\end{array}$ & $\begin{array}{l}n \\
n\end{array}$ & 58 & $\begin{array}{l}-a \\
\therefore i=\end{array}$ & $n$ & \\
\hline
\end{tabular}

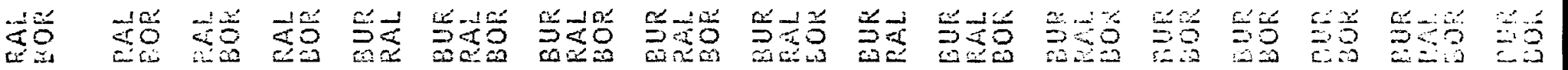

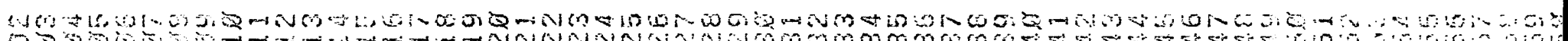
S. man m, 


\begin{tabular}{|c|c|c|c|c|c|c|c|c|c|c|c|c|c|c|c|}
\hline & $\begin{array}{l}c: 0 \\
: \vdots:\end{array}$ & 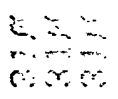 & $\approx \cdots$ & 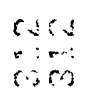 & $\begin{array}{l}63: 2 \\
6 \% \\
6 \%\end{array}$ & $\begin{array}{l}\cdots \\
\cdots \\
0\end{array}$ & $\begin{array}{l}4: 26 \\
6 \\
6\end{array}$ & $\because \therefore$ & 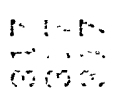 & $\begin{array}{l}\sin 60 \\
\cdots=0 \\
6: 50\end{array}$ & 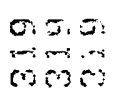 & mis & $\begin{array}{ll}m & -m \\
r & =\end{array}$ & 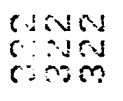 & $\begin{array}{l}\text { mas } \\
\text { mas }\end{array}$ \\
\hline 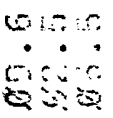 & 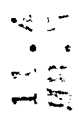 & 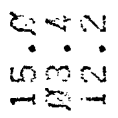 & $\underbrace{0: 0}_{n=1}$ & $\begin{array}{l}\because \div \\
\because \vdots \\
-8\end{array}$ & $\begin{array}{l}: 5: 5 \\
\dot{5}: 5\end{array}$ & $\begin{array}{l}8:-2 \\
m a x\end{array}$ & 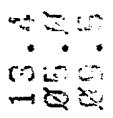 & $\begin{array}{r}-4 \\
-10 \\
-i x\end{array}$ & $\begin{array}{l}\therefore 5: \\
m: 5= \\
m \dot{0}=\end{array}$ & 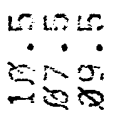 & 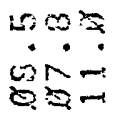 & $\begin{array}{l}10: \\
\dot{0}: \dot{0}\end{array}$ & 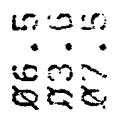 & 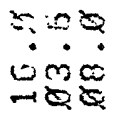 & $\begin{array}{l}0.3 \\
* \therefore:\end{array}$ \\
\hline
\end{tabular}

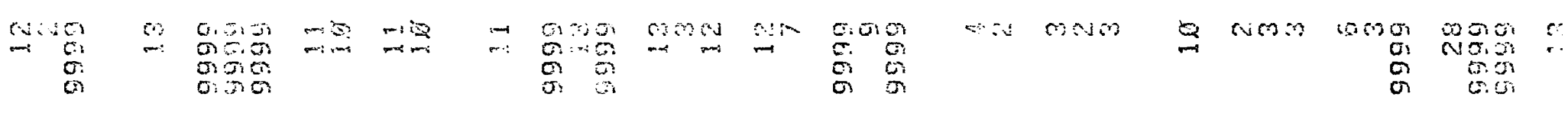

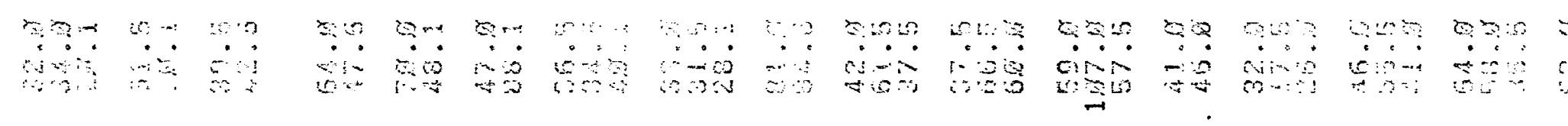

\begin{tabular}{|c|c|c|c|c|c|c|c|c|c|c|c|c|c|c|c|}
\hline 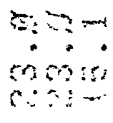 & $\begin{array}{l}0: 3 \\
\because: \infty\end{array}$ & 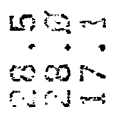 & $\begin{array}{l}30 \\
=\pi \\
\infty\end{array}$ & $\begin{array}{l}3 i-1 \\
\because 3 i= \\
6:=\end{array}$ & $\begin{array}{l}8-1 \\
6 \\
5 \\
3\end{array}$ & $\begin{array}{l}6: S:-1 \\
0:-1 \\
\therefore: 3=-1\end{array}$ & 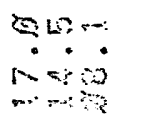 & 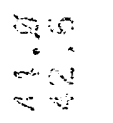 & 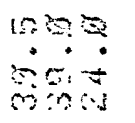 & 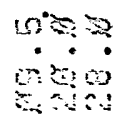 & $\begin{array}{l}\mathrm{s}: 0 \\
\mathrm{c}: 3 \mathrm{i}\end{array}$ & $\begin{array}{l}0: 3 \\
0: 3 \\
0.3\end{array}$ & $\begin{array}{l}3: 5: \\
80 ; \\
-50\end{array}$ &  & 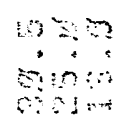 \\
\hline 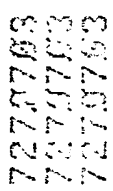 & 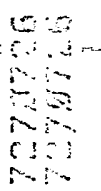 & 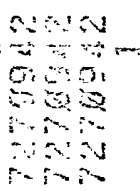 & 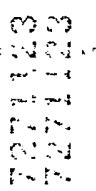 & 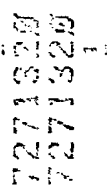 & 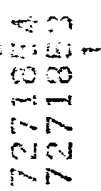 & 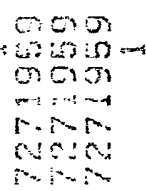 & 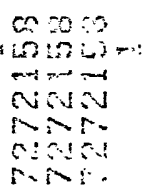 & 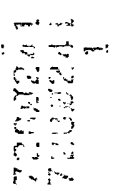 & 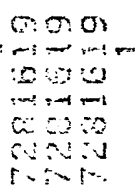 & 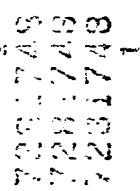 & 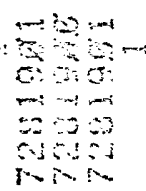 & 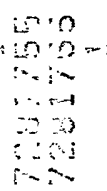 & 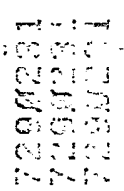 & 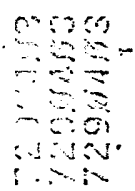 & 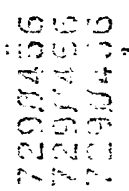 \\
\hline 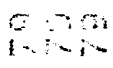 & 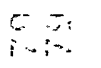 & $\bar{a}=\bar{a}$ & 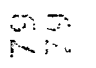 & $\pi n$ & 85 & $\because$ & & & & & & 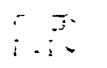 & & & $\because$ \\
\hline
\end{tabular}

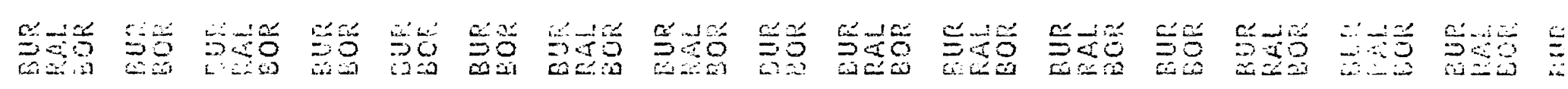
m

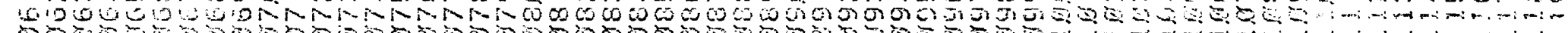
จ 


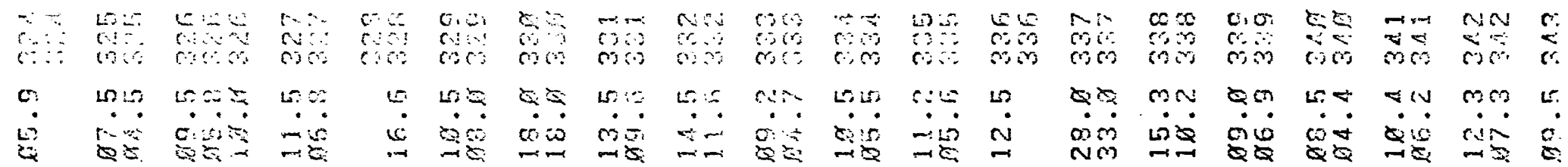

\begin{tabular}{|c|c|c|c|c|c|c|c|c|c|c|c|c|c|c|c|c|c|c|c|}
\hline 50 & 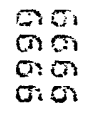 & $\begin{array}{l}\sigma \\
0 \\
0 \\
0\end{array}$ & $\begin{array}{l}0 \\
0 \\
0 \\
0\end{array}$ & $\begin{array}{ll}\cos \\
\mathrm{s}\end{array}$ & $\begin{array}{l}N= \\
N \rightarrow\end{array}$ & $\begin{array}{c}\infty 0 \\
6 \mathrm{n} \\
\mathrm{n} \\
\mathrm{n}\end{array}$ & $\begin{array}{c}\sigma a \\
N a \\
\sigma \\
\sigma\end{array}$ & $\begin{array}{l}\text { no } \\
\text { no } \\
\text { o }\end{array}$ & UE & $\begin{array}{l}\text { No } \\
\text { is }\end{array}$ & $+\infty$ & $\begin{array}{c}\omega \infty \\
\therefore \sigma \\
0 \\
0\end{array}$ & $\begin{array}{l}\sigma: \pi \\
\infty \sigma \\
0 . \\
\pi\end{array}$ & $\begin{array}{l}0 \\
01 \\
0 \\
01\end{array}$ & & & $\begin{array}{l}\text { ni } \\
\text { on } \\
\text { on }\end{array}$ & $\begin{array}{l}0,10 \\
0 \\
\text { b } \\
0\end{array}$ & 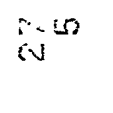 \\
\hline
\end{tabular}

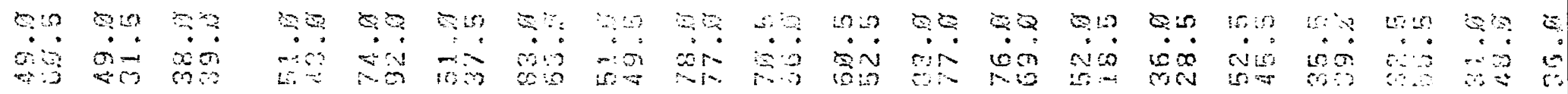

\begin{tabular}{|c|c|c|c|c|c|c|c|c|c|c|c|c|c|c|c|c|c|c|}
\hline 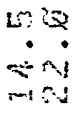 & $\begin{array}{l}30 \\
4 m \\
+i=1\end{array}$ & 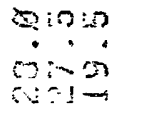 & $\begin{array}{l}\text { sin } \\
n=i \\
8 i n\end{array}$ & $\begin{array}{l}8: ? \\
0: 3 \\
m: 8\end{array}$ & $\begin{array}{l}x: 5 \\
0 \\
0+\infty\end{array}$ & $\begin{array}{l}30 \\
030 \\
0.0\end{array}$ & $\begin{array}{l}640 \\
405 \\
603\end{array}$ & $\begin{array}{l}30 \\
0 \\
0 \\
0\end{array}$ & $\begin{array}{l}\because 0 \\
\because a \\
\because \cdots\end{array}$ & $\begin{array}{l}=3 \\
=\dot{3} \\
=\dot{1}\end{array}$ & $\begin{array}{l}0 \% \\
0 ; \\
03\end{array}$ & $\begin{array}{l}0: 0 \\
0: 0 \\
0: 5\end{array}$ & $\begin{array}{l}0: 0 \\
i: \pi\end{array}$ & $\begin{array}{l}=n \\
0 \\
0 \\
0\end{array}$ & $\begin{array}{l}5: 5 \\
\therefore=8 \\
30\end{array}$ & $\begin{array}{l}3: 9 \\
0 \\
0 \\
0\end{array}$ & $\begin{array}{l}4: 9 \\
: 0 \\
\therefore: ?\end{array}$ & $\begin{array}{l}\text { sin } \\
\therefore-i n \\
\text { in }\end{array}$ \\
\hline $\begin{array}{l}6 \\
0 \\
0 \\
01 \\
01\end{array}$ & 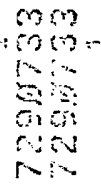 & 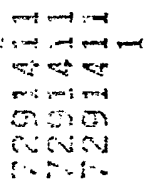 & $\begin{array}{l}\infty: 9 \\
m=1 \\
m i s \\
m i s\end{array}$ & 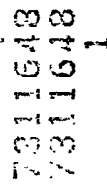 & 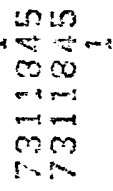 & 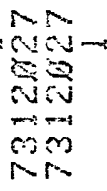 & 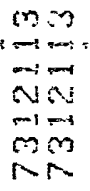 & 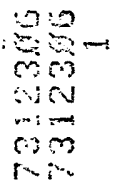 & 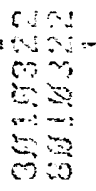 & $\begin{array}{l}3=1 \\
3 i 5 \\
30 \\
60 \\
50 \\
50\end{array}$ & 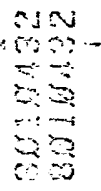 & 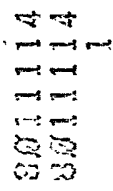 & 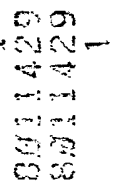 & 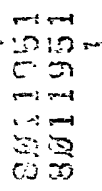 & 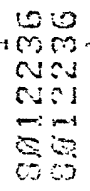 & 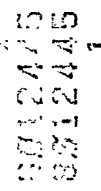 & 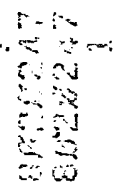 & 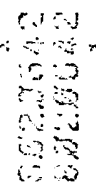 \\
\hline
\end{tabular}

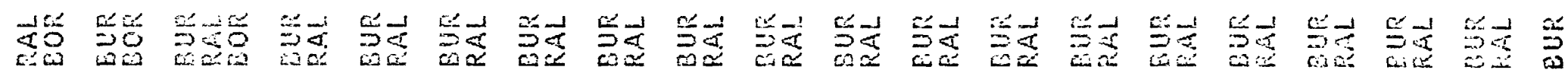

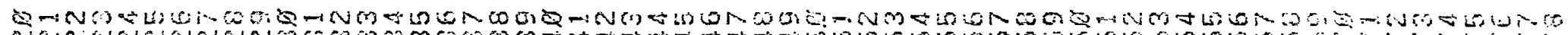

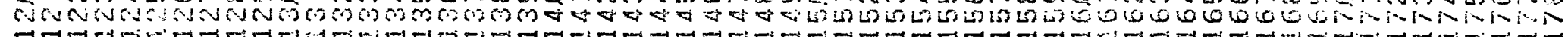

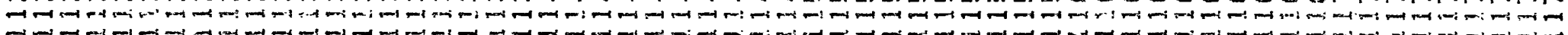




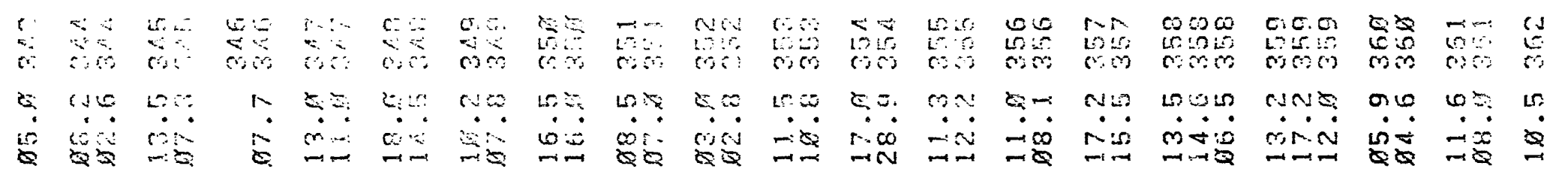

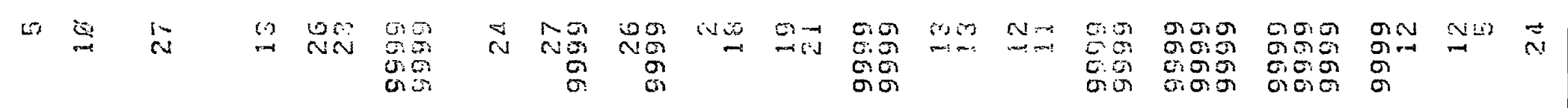

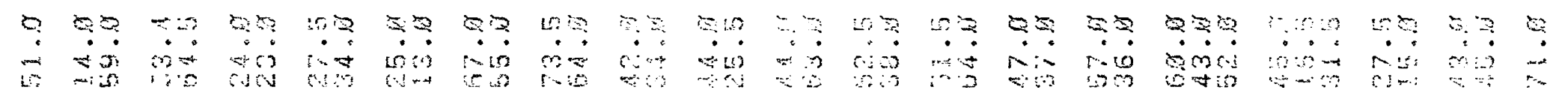

\begin{tabular}{|c|c|c|c|c|c|c|c|c|c|c|c|c|c|c|c|c|c|c|}
\hline 8 & $\begin{array}{l}50 \\
30 \\
34\end{array}$ & 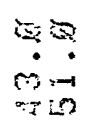 & $\begin{array}{l}80 \\
80 \\
80\end{array}$ & $\begin{array}{l}0: 3 \\
98\end{array}$ & $\begin{array}{l}a s \\
0\end{array}$ & an & 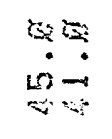 & 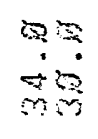 & 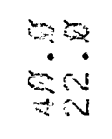 & 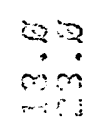 & $\because \rightarrow$ & $\begin{array}{l}\operatorname{ma} \\
\min \end{array}$ & 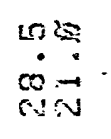 & $\begin{array}{l}30 \\
0 \\
30\end{array}$ & 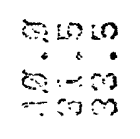 & 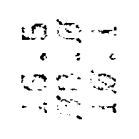 & $\begin{array}{l}5: 4 \\
\vdots 0 \\
0\end{array}$ & $\begin{array}{l}0 ? \\
3: 3 \\
23\end{array}$ \\
\hline & 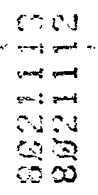 & 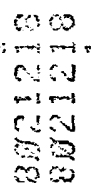 & $\begin{array}{l}05 \\
85 \\
53 \\
85 \\
65\end{array}$ & 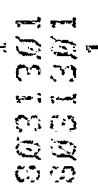 & $\begin{array}{l}50 \\
20 \\
30 \\
\cdots \\
6 \\
6\end{array}$ & $\begin{array}{l}15: 5 \\
: 5 \\
0 \\
0 \\
0 \\
0\end{array}$ & 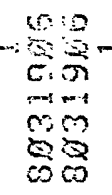 & 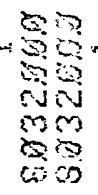 & $\begin{array}{l}5 \\
20 \\
20 \\
03\end{array}$ & $\begin{array}{l}\infty \\
80 \\
60\end{array}$ & 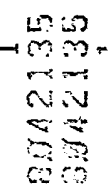 & $\$ 3$ & 60 & & $\begin{array}{l}-50 \\
68 \\
08\end{array}$ & $\begin{array}{l}6 \\
6 \% \\
6\end{array}$ & & 8 \\
\hline & $\tilde{F}$ & 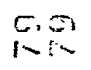 & $=$ & $\therefore$ & sin & 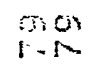 & ara & 8 & & & $M$ & & 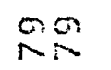 & & & & & \\
\hline
\end{tabular}

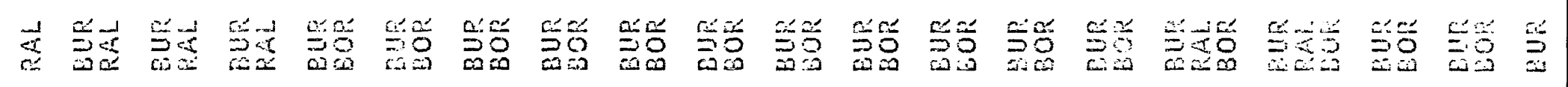

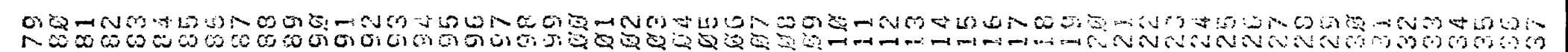

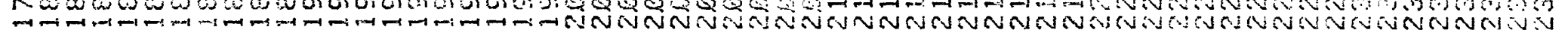
개 


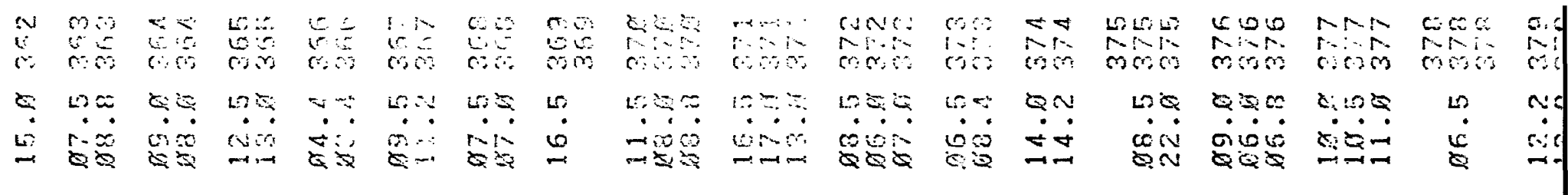

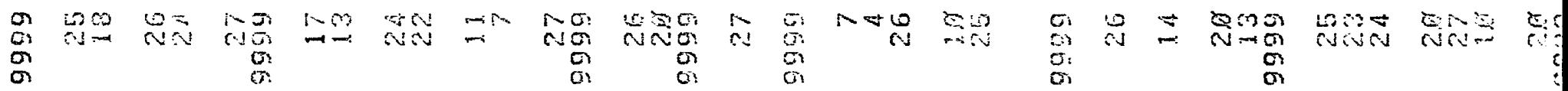

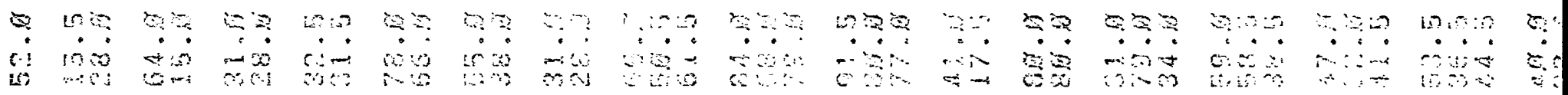

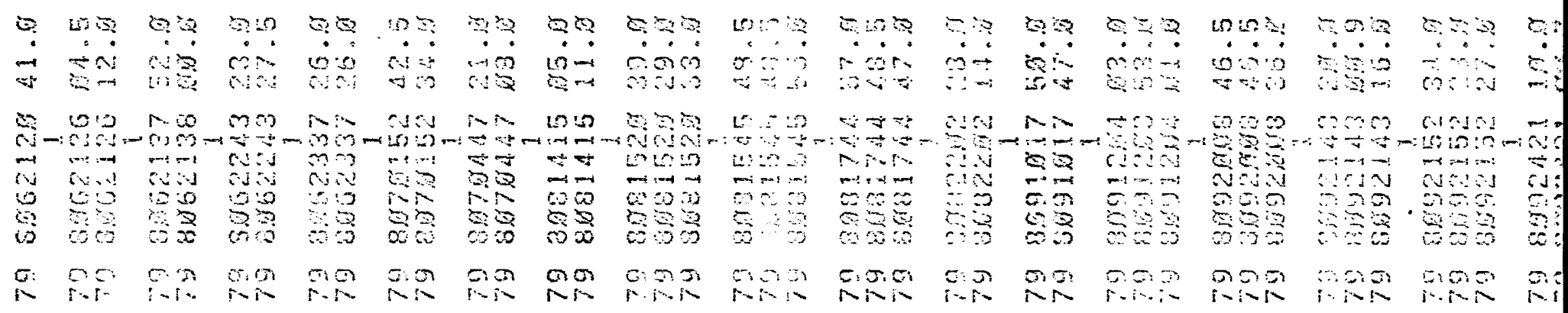

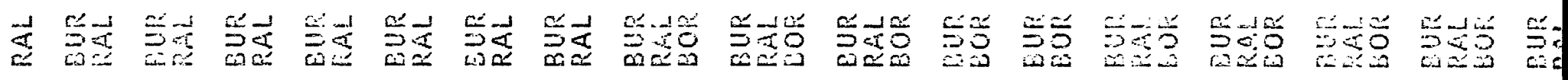

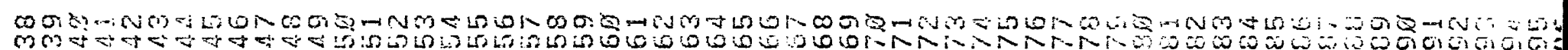

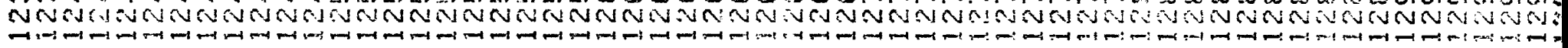




\begin{tabular}{|c|c|c|c|c|c|c|c|c|c|c|c|c|c|c|}
\hline 6 & \begin{tabular}{l}
$2=2$ \\
\hdashline \\
0
\end{tabular} & 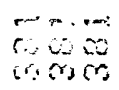 & $\begin{array}{l}6 \\
6 \\
6 \\
0\end{array}$ & 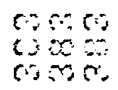 & 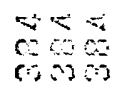 & 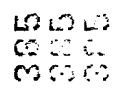 & 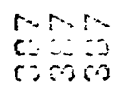 & $\begin{array}{l}c=0 \\
\cos \theta \\
\cos \theta\end{array}$ & 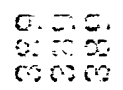 & 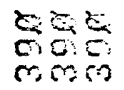 & 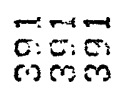 & 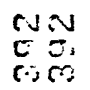 & $\begin{array}{l}\mathrm{s} s=0 \\
\mathrm{~s}=\mathrm{m}\end{array}$ & $\begin{array}{l}x \leq \neq \\
6 \pm 0 \\
0\end{array}$ \\
\hline $\begin{array}{l}\infty \\
\dot{z}\end{array}$ & 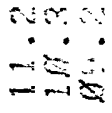 & $\dot{8}$ & 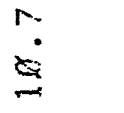 & $\begin{array}{l}\text { जE: } \\
\dot{4} \dot{0}\end{array}$ & $\begin{array}{l}\because 2 \\
00\end{array}$ & 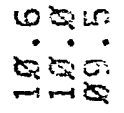 & 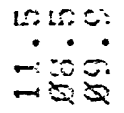 & 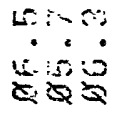 & 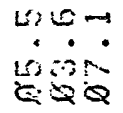 & 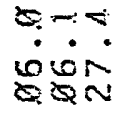 & $\begin{array}{l}n+N \\
0 \sin \end{array}$ & $\begin{array}{l}\dot{Q}: 0 \\
\dot{0}: \dot{0}\end{array}$ & $\begin{array}{l}8: 6 \\
810\end{array}$ & $\begin{array}{r}x+5: 0 \\
\dot{m}=0\end{array}$ \\
\hline
\end{tabular}
$m$ nivis

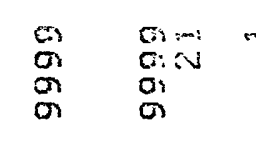

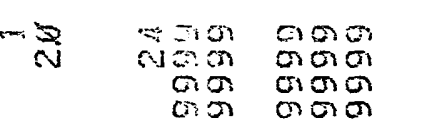

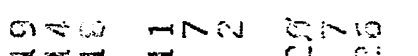

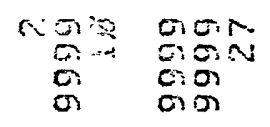
Lo:

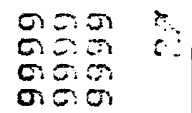

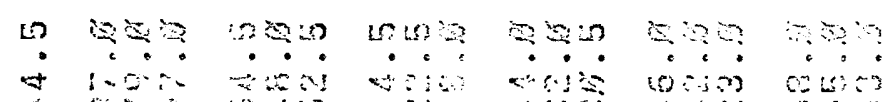

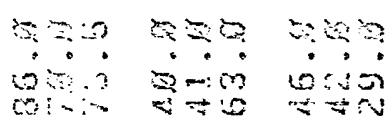
enis
in: 200
in $\sin$ $\sin$
20
mo

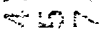

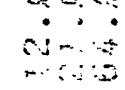
a

\begin{tabular}{|c|c|c|c|c|c|c|c|c|c|c|c|c|c|c|c|}
\hline vis & 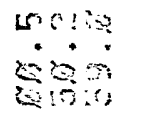 & 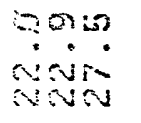 & 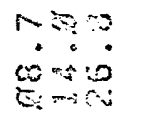 & 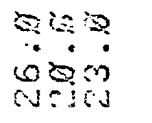 & 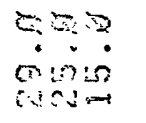 & 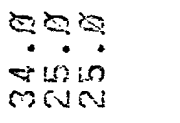 & 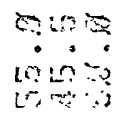 & 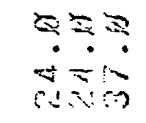 & 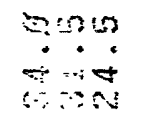 & $\begin{array}{l}6=0 \\
c: 00 \\
\infty=0\end{array}$ & 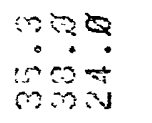 & $\begin{array}{r}30 \\
-3 \\
-3\end{array}$ & 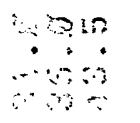 & 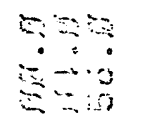 & 8 \\
\hline 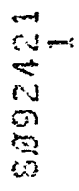 & 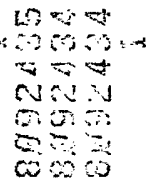 & 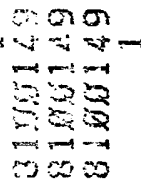 & 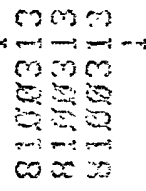 & 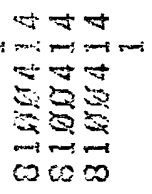 & 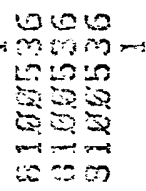 & 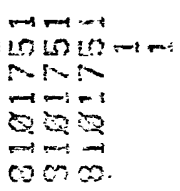 & 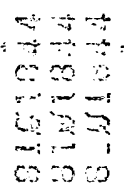 & 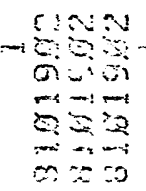 & 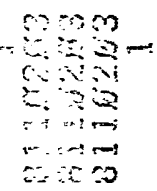 & 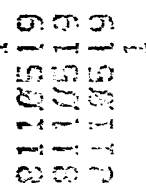 & 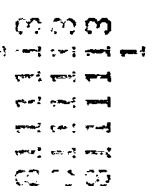 & 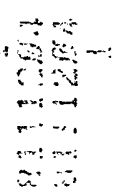 & 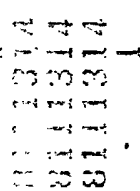 & 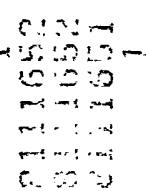 & $i=$ \\
\hline
\end{tabular}

○

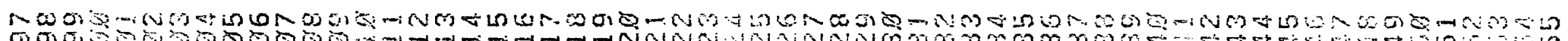

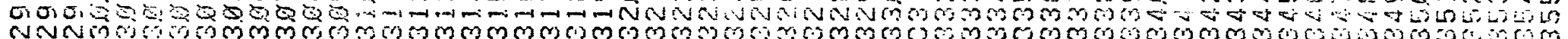
n- 


\begin{tabular}{|c|c|c|c|c|c|c|c|c|c|c|c|c|c|c|c|}
\hline $\begin{array}{l}4=5 \\
620\end{array}$ & 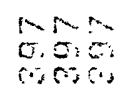 & $\begin{array}{ll}c: \\
c: c\end{array}$ & $\begin{array}{l}00 \\
00 \\
\cos 0\end{array}$ & $\varepsilon:-2$ & $\begin{array}{ll}x \\
x<\end{array}$ & 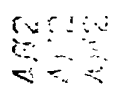 & $E$ & 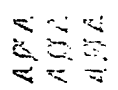 & L & $\underset{4}{5}$ & 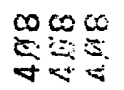 & 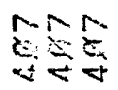 & 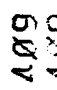 & $\begin{array}{l}1:= \\
\vdots\end{array}$ & $C_{n}$ \\
\hline 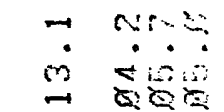 & 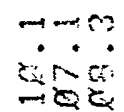 & $\dot{0}$ & & $\begin{array}{l}\dot{0} \\
\dot{m}\end{array}$ & 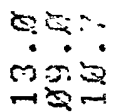 & 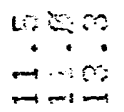 & 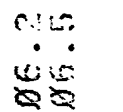 & $\dot{v i s}$ & $\begin{array}{l}x-10 \\
00 \\
00\end{array}$ & $\begin{array}{l}m= \\
103 \\
08\end{array}$ & $\dot{0}$ & $\stackrel{5}{\square}$ & $\stackrel{\infty}{\infty}$ & $\dot{0}$ & \\
\hline
\end{tabular}

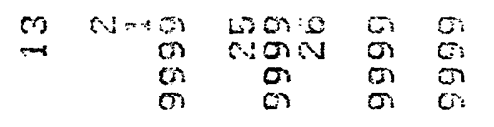

\begin{tabular}{|c|c|c|}
\hline & 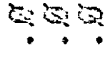 & 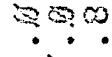 \\
\hline 0 & 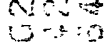 & $83=$ \\
\hline
\end{tabular}

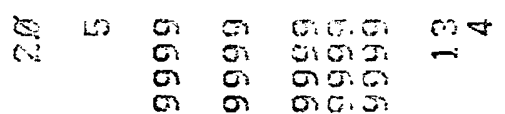

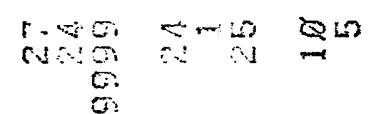

Gis

Cis

Gion cis:

a $\sigma: 0$

Gois

ก)

$\therefore$

\begin{tabular}{|c|c|c|c|c|c|c|c|c|c|c|c|c|c|c|c|}
\hline $\begin{array}{l}830 \\
4 i \pi\end{array}$ & $\begin{array}{l}m: 5 \\
\because m 0 \\
\because 28\end{array}$ & 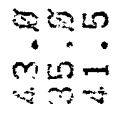 & 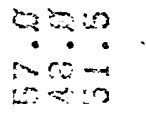 & $\begin{array}{l}0: 8 \\
010 \\
03\end{array}$ & 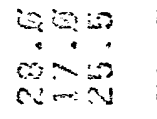 & 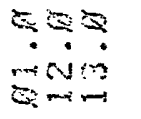 & 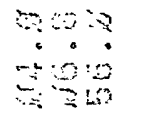 & $\begin{array}{r}0603 \\
25: 2 \\
4: 3\end{array}$ & $\begin{array}{r}15: 9 \\
\div \div 3 \\
4: 3\end{array}$ & $\begin{array}{l}=3 \\
=-4 \\
=-4\end{array}$ & $\begin{array}{l}5:-0 \\
\therefore-i\end{array}$ & $\begin{array}{l}10010 \\
0509 \\
\because 10 \%\end{array}$ & 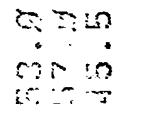 & 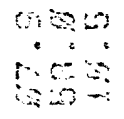 & $\begin{array}{l}-5: 3 \\
\therefore: \\
\therefore:\end{array}$ \\
\hline 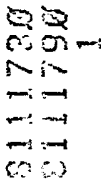 & 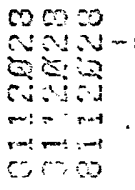 & 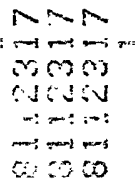 & 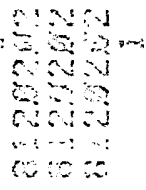 & 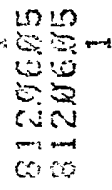 & 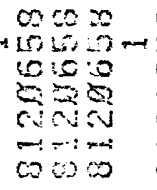 & 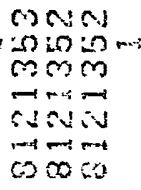 & 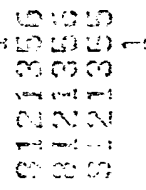 & 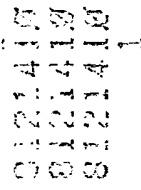 & 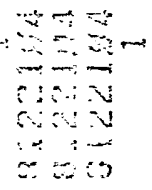 & 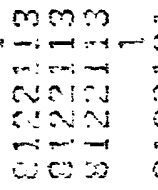 & 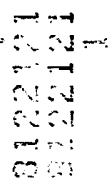 & 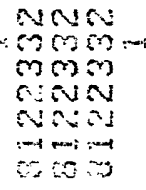 & 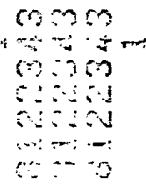 & 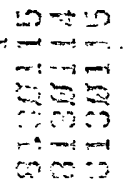 &  \\
\hline $\begin{array}{ll}c \\
r \times n\end{array}$ & 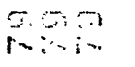 & $\begin{array}{l}05 \\
10 i=1\end{array}$ & $\begin{array}{l}6 \\
M\end{array}$ & $\begin{array}{l}\text { Cin } \\
\text { N. }\end{array}$ & MN & and & 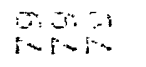 & $\begin{array}{lll}n \\
i \rightarrow n\end{array}$ & son & & Si & $\begin{array}{lll}0 & 100 \\
n+m\end{array}$ & & 小心 & \\
\hline
\end{tabular}

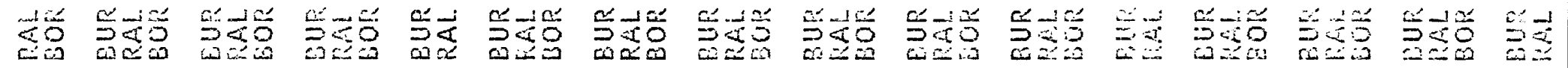

5N

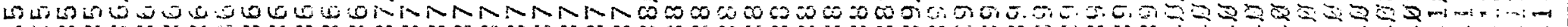

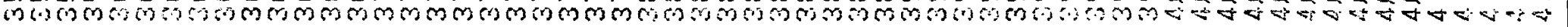

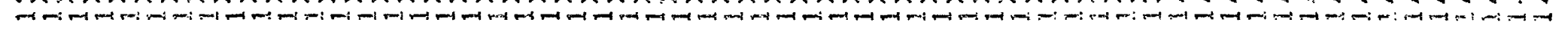




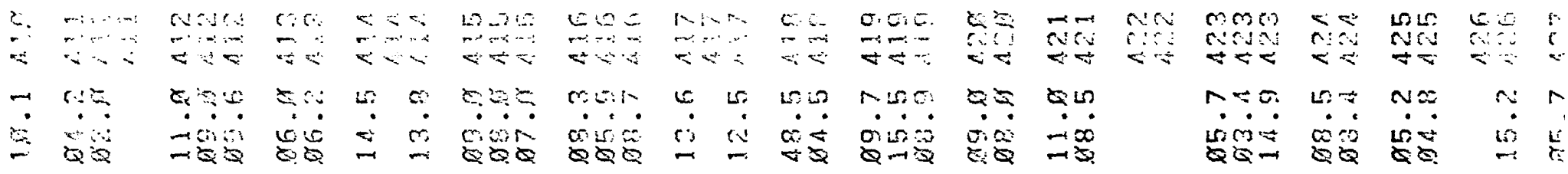



๓⿴囗十

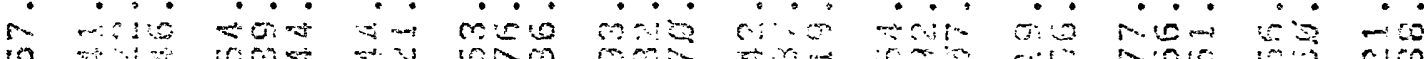

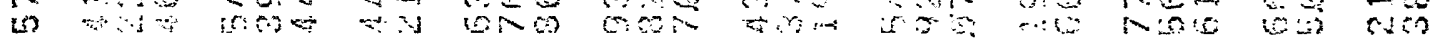

\begin{tabular}{|c|c|c|c|c|c|c|c|c|c|c|c|c|c|c|c|c|}
\hline $\begin{array}{l}\infty \\
\infty\end{array}$ & 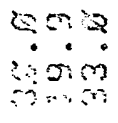 & 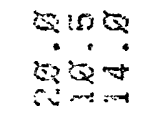 & 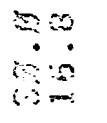 & 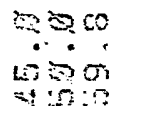 & $\begin{array}{l}300 \\
8-\infty\end{array}$ & 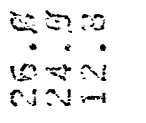 & 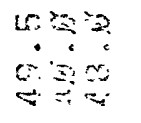 & $\begin{array}{l}03 \\
\vdots \\
\vdots \\
\vdots \\
\vdots\end{array}$ & 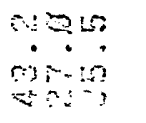 & $\begin{array}{l}\because=0 \\
\because \approx \\
\because y\end{array}$ & $\begin{array}{l}0: 5 \\
: 5 \\
5: 2\end{array}$ & $\begin{array}{l}3: 2 \\
0 \div \\
30\end{array}$ & $\begin{array}{l}15: 0 \\
-60 \\
0: 5: 5\end{array}$ & $\begin{array}{l}3: 3 \\
\therefore \therefore \\
\therefore\end{array}$ & $\begin{array}{l}5: \infty \\
: 5 \\
2: 5\end{array}$ & $\begin{array}{l}10: 2 \\
\therefore: 3 \\
\therefore: 3\end{array}$ \\
\hline 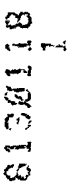 & 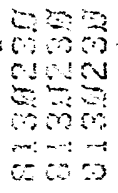 & 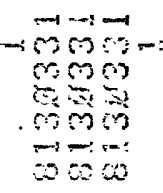 & 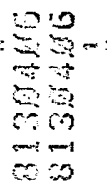 & 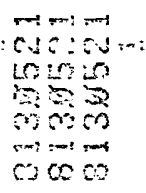 & 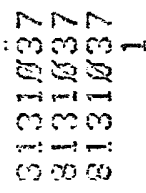 & 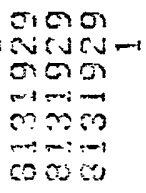 & 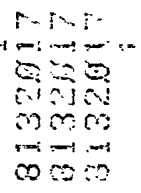 & 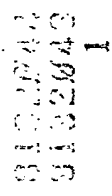 & 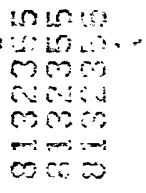 &  & $\begin{array}{l}-\infty \\
200 \\
=0 \\
=0 \\
=0 \\
=0\end{array}$ & 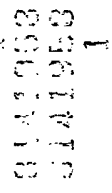 & 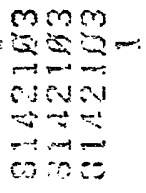 & 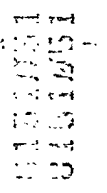 & 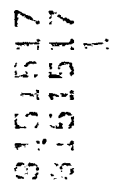 & 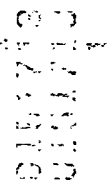 \\
\hline & 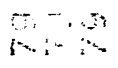 & $\begin{array}{c}G i n \\
M M N\end{array}$ & $0: 3$ & $\begin{array}{ll}\infty \\
1 . \infty\end{array}$ & G6 & ind & $\begin{array}{l}0.0 \\
r .1\end{array}$ & $\begin{array}{l}=0 \\
i 2: 0\end{array}$ & Disin & & & & 3 & $\begin{array}{l}a=i \\
0 . i=\end{array}$ & & \\
\hline
\end{tabular}




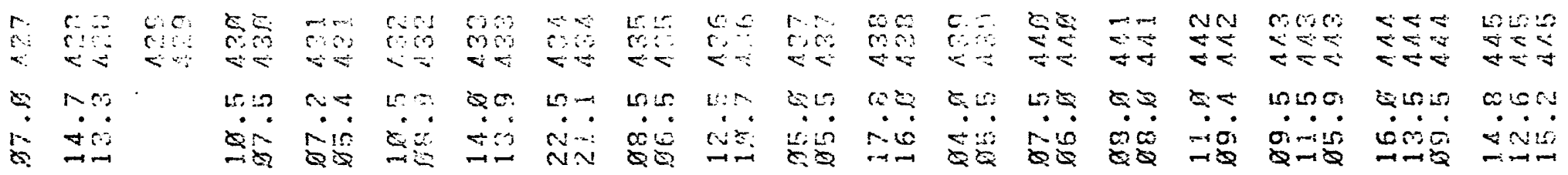

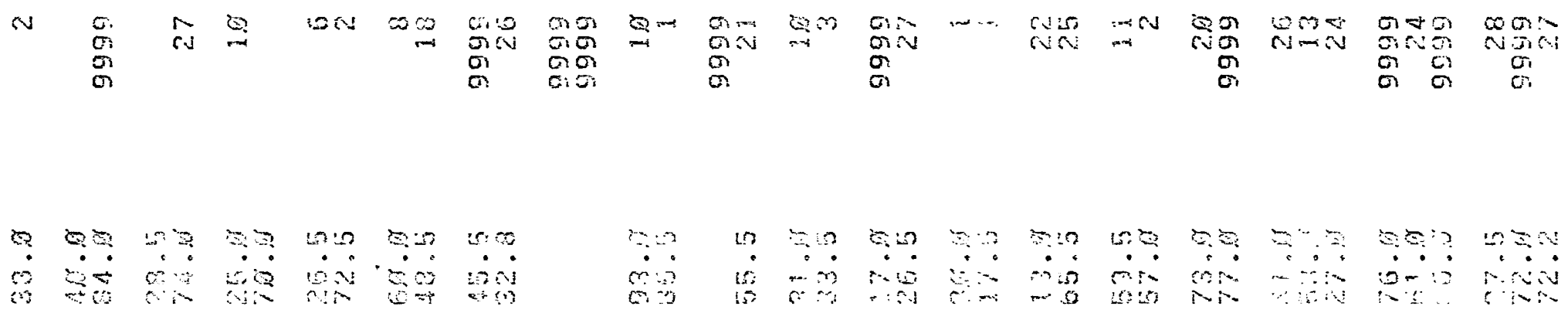

\begin{tabular}{|c|c|c|c|c|c|c|c|c|c|c|c|c|c|c|c|c|c|c|}
\hline$\ddot{0}$ & $\begin{array}{l}1=: 2 \\
0: 0 \\
5: 5\end{array}$ & $\begin{array}{l}x=2 \\
\therefore=2\end{array}$ & $\begin{array}{l}-i 0 \\
\dot{5}: \dot{0}\end{array}$ & $\begin{array}{l}60 \\
\div 0 \\
80\end{array}$ & $\begin{array}{l}\because 8 \\
\therefore i\end{array}$ & 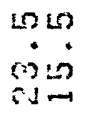 & $\begin{array}{l}3 \pi \\
3 \pi \\
03\end{array}$ & $\begin{array}{l}30 \\
\div 20 \\
100\end{array}$ & $\begin{array}{l}3: 3 \\
\therefore \infty\end{array}$ & 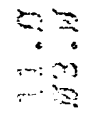 & 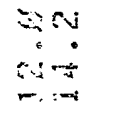 & 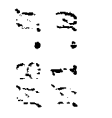 & $\begin{array}{c}1010 \\
510 \\
30\end{array}$ & 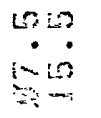 & $\begin{array}{l}-3: 2 \\
-: 0 \\
-: 3\end{array}$ & 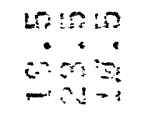 & 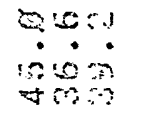 & 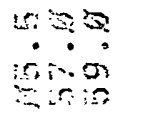 \\
\hline $\begin{array}{l}3 \\
6 \\
0 \\
0 \\
0\end{array}$ & 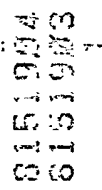 & 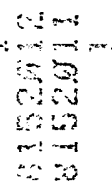 & $\begin{array}{l}-8 \\
08 \\
06 \\
04 \\
00\end{array}$ & 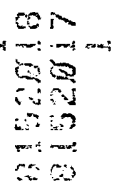 & 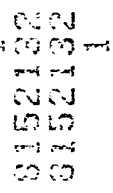 & 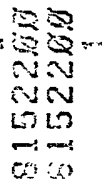 & 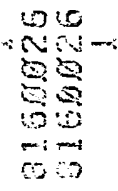 & 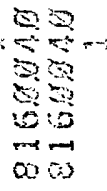 & 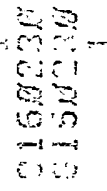 & 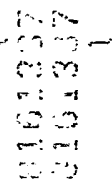 & 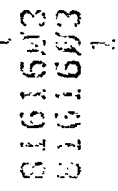 & 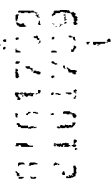 & 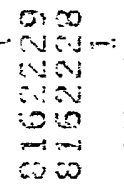 & 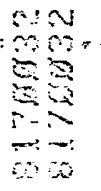 & $\begin{array}{l}50 \\
10 \leq 2 \\
1=0 \\
1 \rightarrow-1 \\
0=3\end{array}$ & 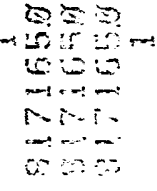 & 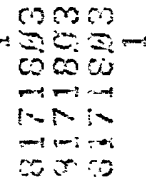 & 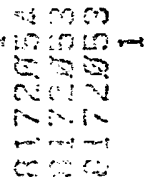 \\
\hline
\end{tabular}

$\because$ 후

世UWH

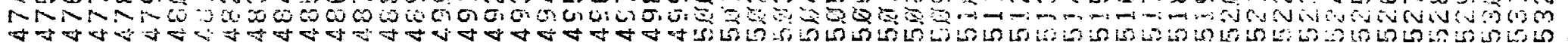

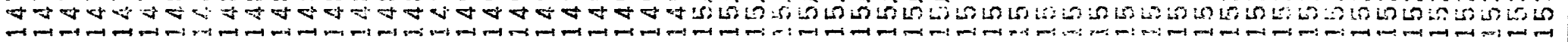




\begin{tabular}{|c|c|c|c|c|c|c|c|c|c|c|c|c|c|c|c|}
\hline $\begin{array}{l}\because+\cdots \\
\because\end{array}$ & 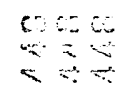 & 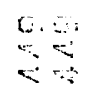 & $\begin{array}{l}25 \\
25: 5 \\
28\end{array}$ & \begin{tabular}{l}
$\cdots$ \\
\hdashline \\
\hdashline
\end{tabular} & 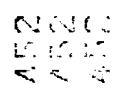 & $\begin{array}{l}5 \\
2\end{array}$ & 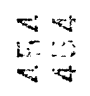 & 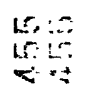 & $\begin{array}{l}0: 3 \\
1:-2\end{array}$ & $\begin{array}{l}N \\
5\end{array}$ & 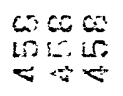 & $\begin{array}{l}c: 0: \\
15: 5 \\
\rightleftarrows:\end{array}$ & $\begin{array}{l}8 \% \\
\varepsilon \\
\varepsilon\end{array}$ & 50 & $\begin{array}{l}0: 0 \\
8: 3\end{array}$ \\
\hline 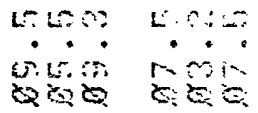 & $\begin{array}{l}\text { L } \\
0 \\
0 \\
0\end{array}$ & 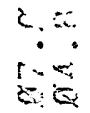 & 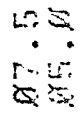 & $\begin{array}{l}0: 0 \\
05 \\
00\end{array}$ & 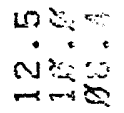 & $\begin{array}{l}c \\
\therefore: 3 \\
8: 3\end{array}$ & $\begin{array}{l}3:= \\
0: 5 \\
0\end{array}$ & $\dot{x}$ & $\begin{array}{l}\dot{\theta} \\
\dot{0} \dot{\theta} \\
\dot{\theta}\end{array}$ & $\begin{array}{r}-10 \\
-20\end{array}$ & 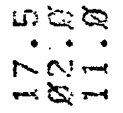 & $\begin{array}{l}x \\
\dot{\alpha} x\end{array}$ & $\begin{array}{l}\propto L: \\
\dot{\sigma} \dot{\varepsilon} \\
\dot{Q} \dot{q}\end{array}$ & $\begin{array}{l}N L \\
\dot{Q}= \\
\dot{Q}=\end{array}$ & $\begin{array}{l}10 \\
\dot{8}:\end{array}$ \\
\hline
\end{tabular}

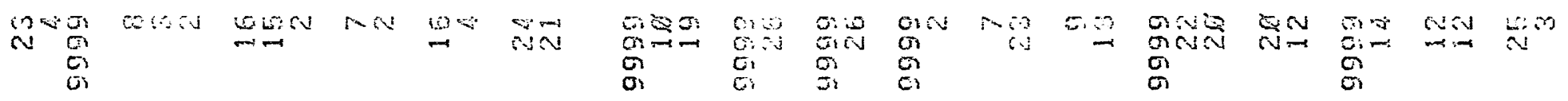

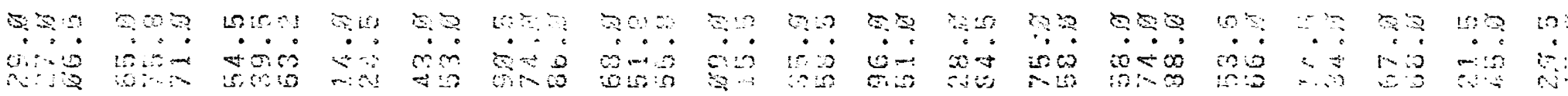

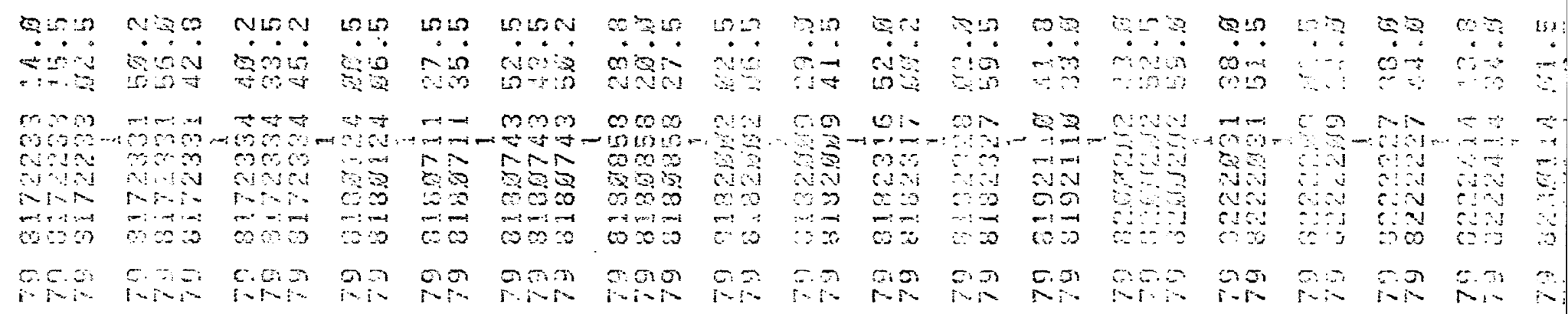

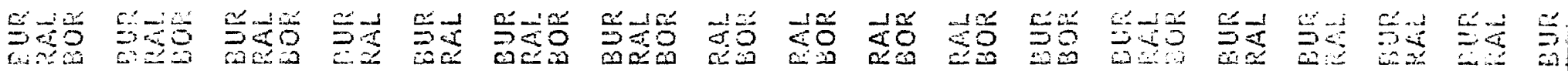

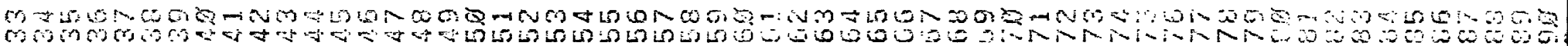

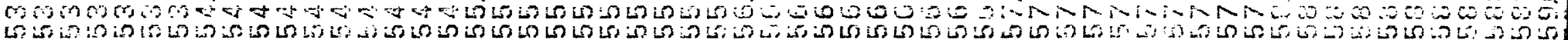
L 


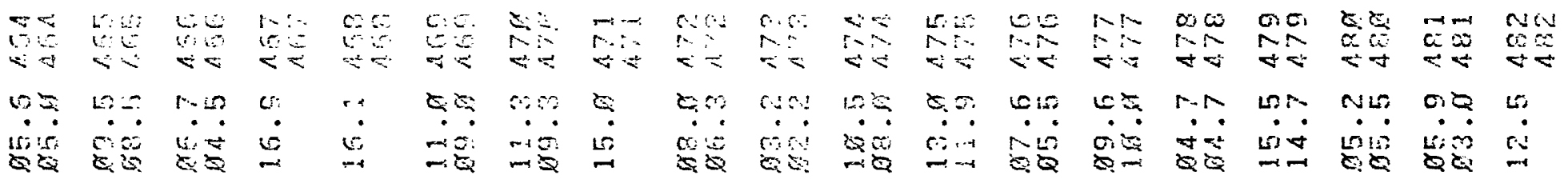

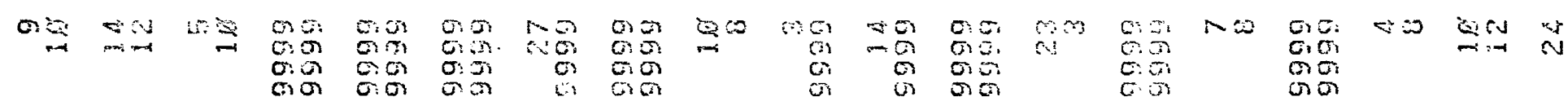

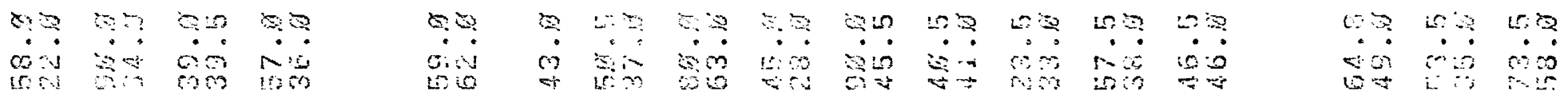

\begin{tabular}{|c|c|c|c|c|c|c|c|c|c|c|c|c|c|c|c|c|c|c|}
\hline $\begin{array}{l}89 \\
86 \\
85\end{array}$ & 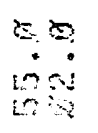 & $\begin{array}{l}i n: ? \\
\infty \\
\infty\end{array}$ & $\begin{array}{l}8 \% \\
80\end{array}$ & 08 & $\begin{array}{l}0 ! 8 \\
0: 3 \\
+15\end{array}$ & $\begin{array}{l}8 \% \\
86 \\
00\end{array}$ & $\dot{\theta}$ & $\ln 5$ & $\begin{array}{l}68 \\
5: 5 \\
\because \vdots !\end{array}$ & 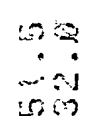 & $\begin{array}{l}03 \\
i=3 \\
0=3\end{array}$ & $\begin{array}{l}120 \\
80\end{array}$ & $\begin{array}{l}08 \\
8 \\
80 \\
48\end{array}$ & 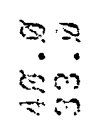 & $\begin{array}{l}8 ? \\
03 \\
015\end{array}$ & 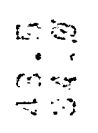 & 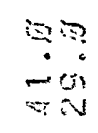 & $\begin{array}{l}93 \\
30 \\
30\end{array}$ \\
\hline 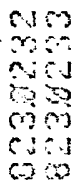 & 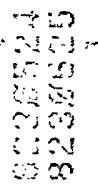 & $\begin{array}{r}-50 \\
90 \\
80 \\
60 \\
0\end{array}$ & 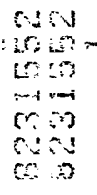 & 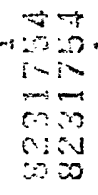 & 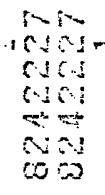 & 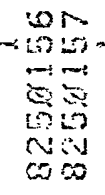 & 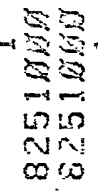 & 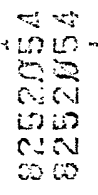 & 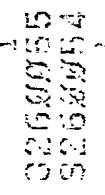 & $\begin{array}{l}50 \\
0.00 \\
60 \\
60 \\
60\end{array}$ & 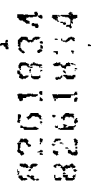 & 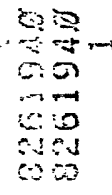 & 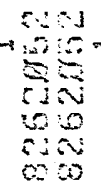 & $\begin{array}{l}30 \\
01 \\
001 \\
000\end{array}$ & 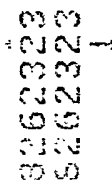 & $\begin{array}{r}0 \\
8 \\
2\end{array}$ & $\begin{array}{r}0 \\
-50 \\
0 \\
6\end{array}$ & 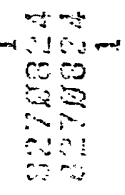 \\
\hline & 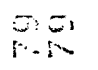 & $\pi R$ & QR & $\infty 2$ & $R \Omega$ & $\pi$ & Sic & no & 82 & $i$ & $\Phi=$ & Din & $n$ & & $\operatorname{lin}$ & $\infty$ & तi & \\
\hline
\end{tabular}

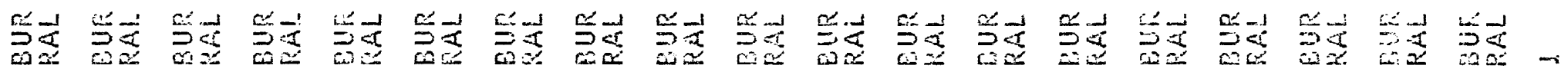

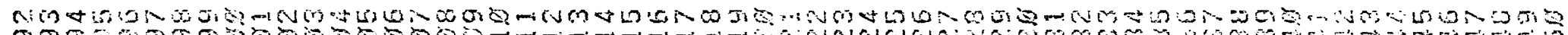

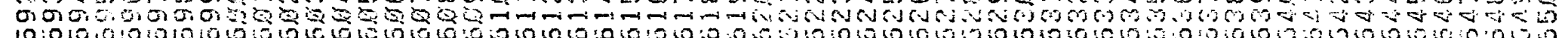

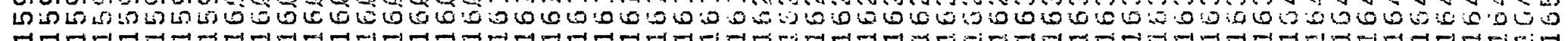


APPENDIX C

3 pages

List of Preliminary Hypocenters 
œ

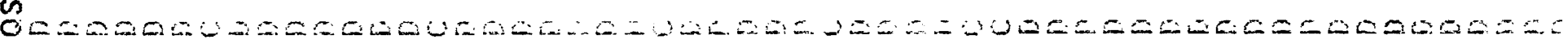

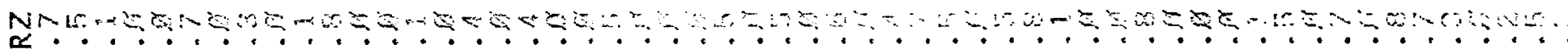

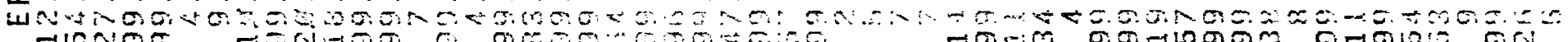

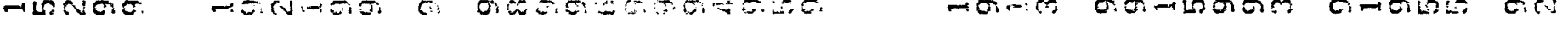

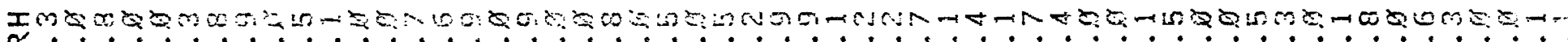

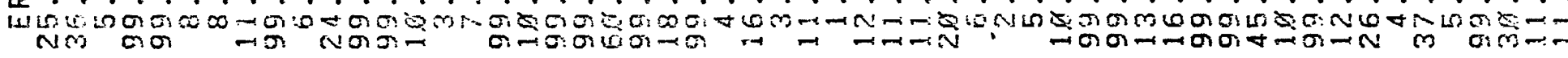

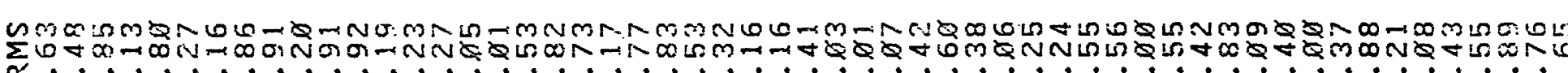

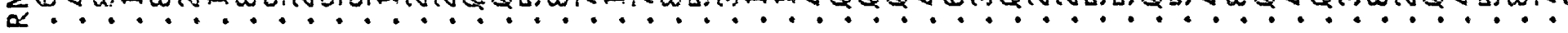
$\rightarrow \rightarrow-\rightarrow$

N

$N \rightarrow$ $\rightarrow-1$

Z E

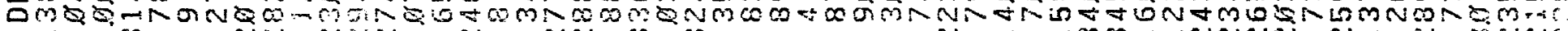

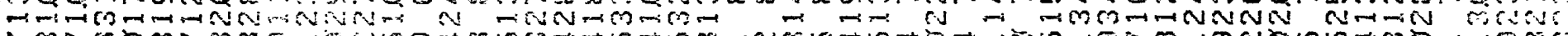
a MMN

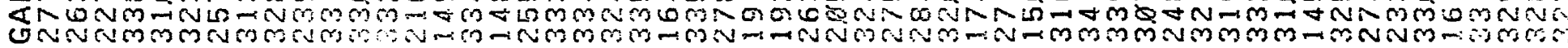
OD $\sum_{\Sigma}^{5}$

INGA

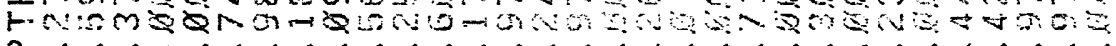

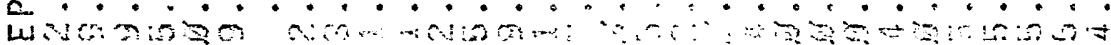
0.1010

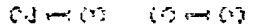

$\rightarrow$

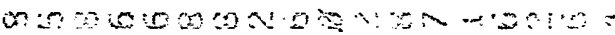
in

3

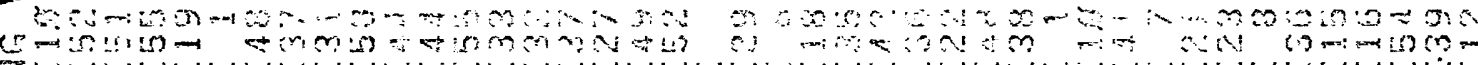

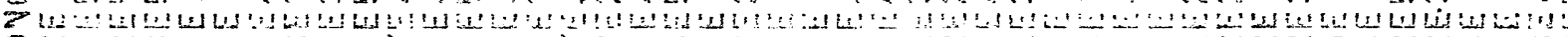

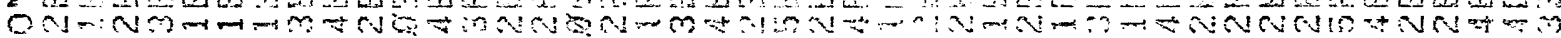

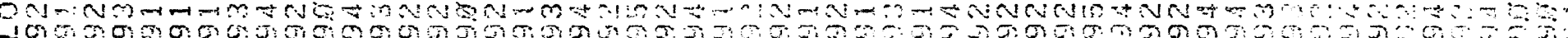

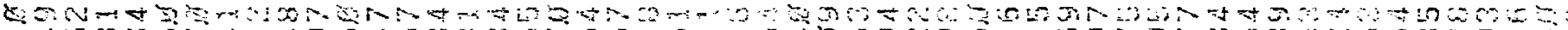
7.

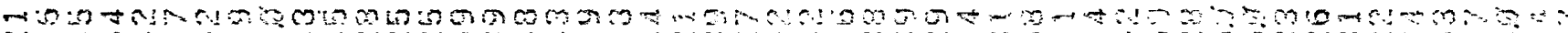
-n.

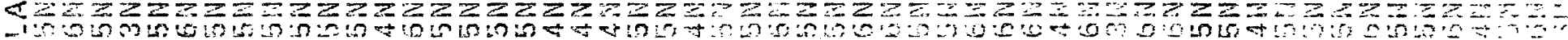

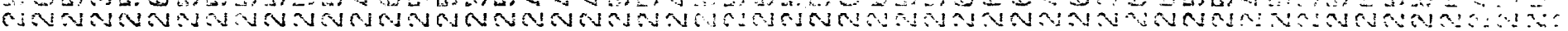

ow mms m

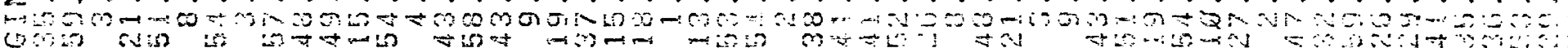

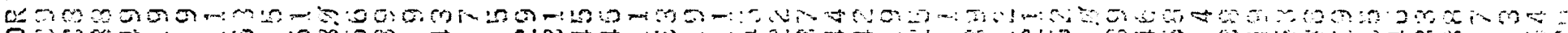
do

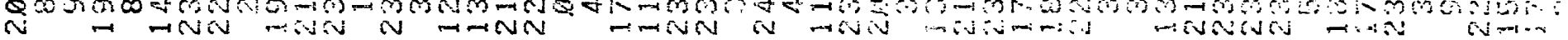

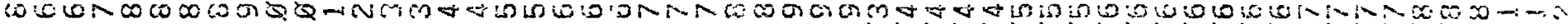

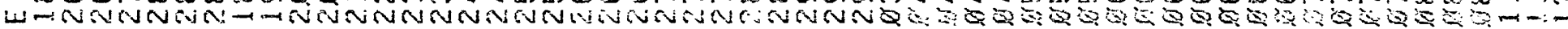

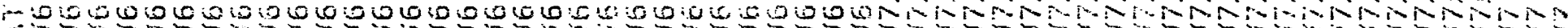

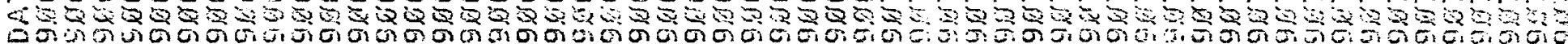

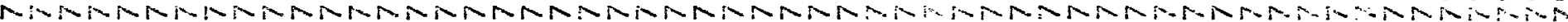

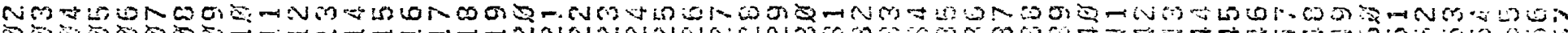

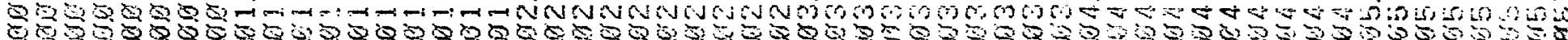
6. 
Ecompt:

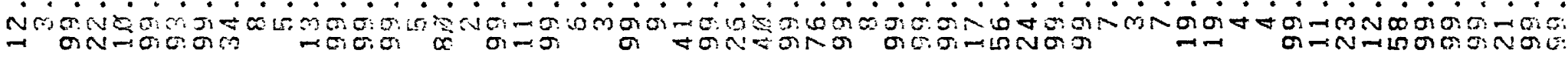

HE-G NStLLNISMLD $\sim$

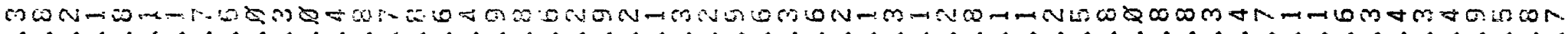

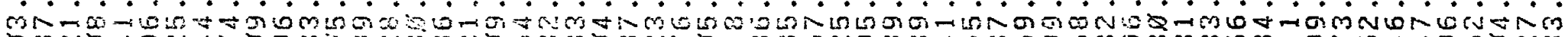

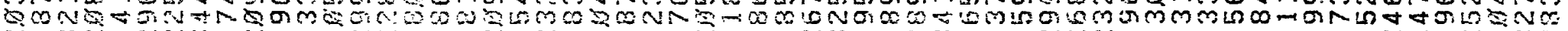

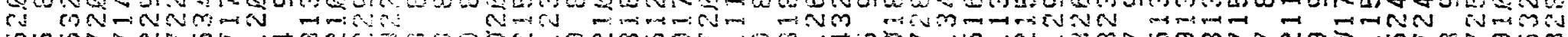

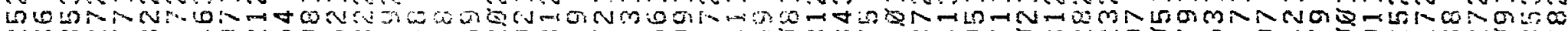



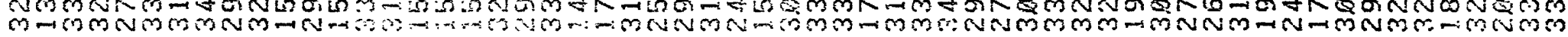

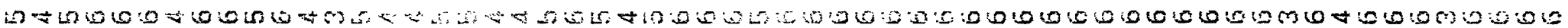

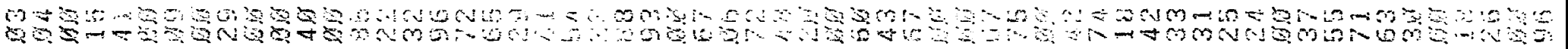
a

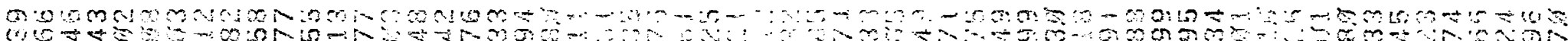

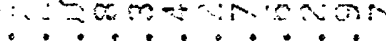

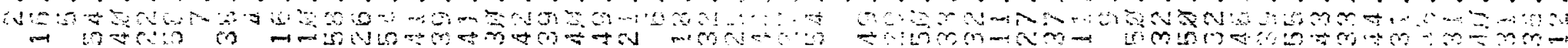
mb he

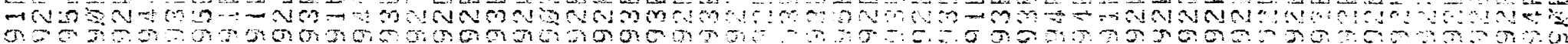
का r 2 m

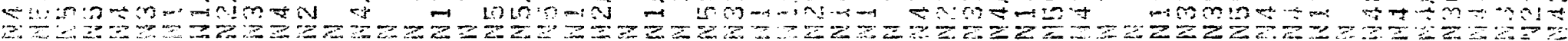
1.

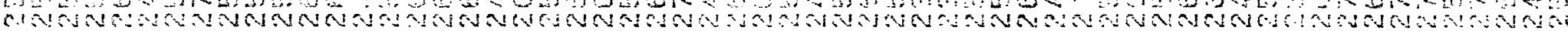

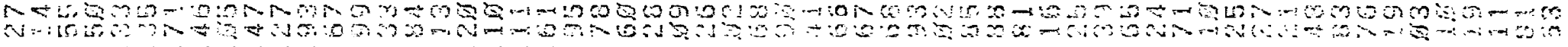

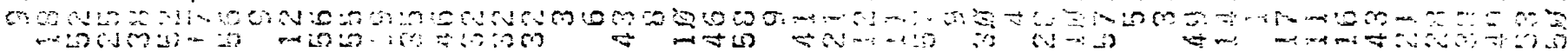

a =

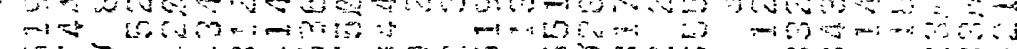
womm-

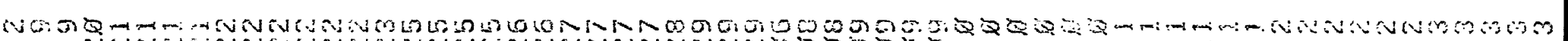

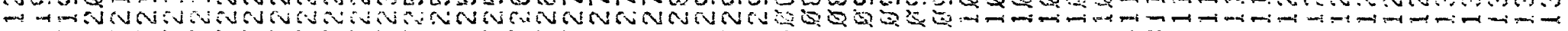

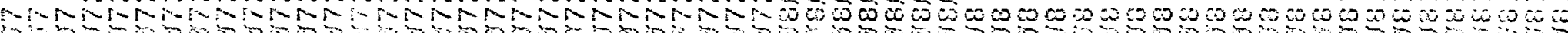
б.

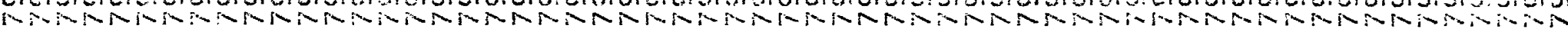

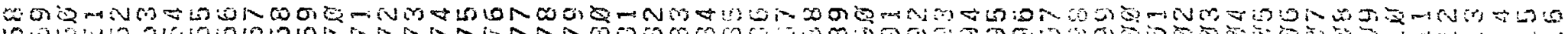

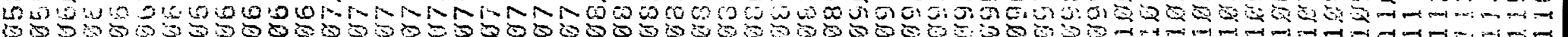

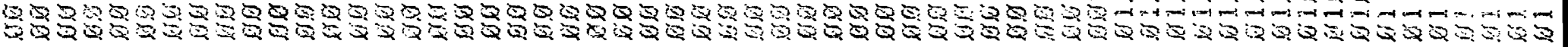




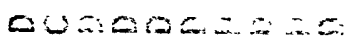
$w:-3=2 \div-\because=$ $\dot{w}=\dot{0} \dot{0} \dot{0} \dot{0} \dot{0}$ osesta:

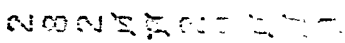
- 0 - $c^{2} \cdot$ n:

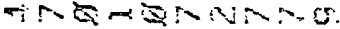
$\cos 5060-2069$
\end{abstract}

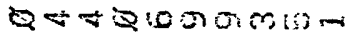
........ 5050300000200

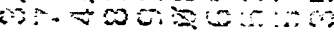
$\rightarrow \quad N N=-1, \ldots+6$

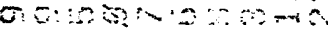

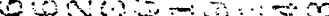
(1) m

$\omega 0: 0: 000.50$

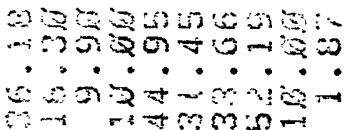

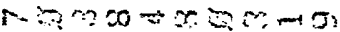
ow- . . . . . ในา

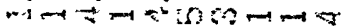

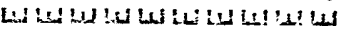

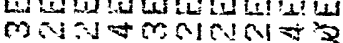

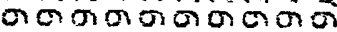

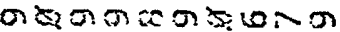
$\infty-100 \%$ inis - . . . . . .

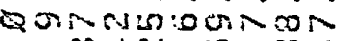
$\Rightarrow-m \forall N=10 m$ $2 \geq \geq 2 \geq \geq 2=2$ NONONNNNAN

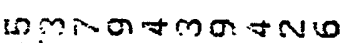

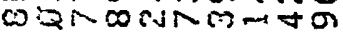
- . . . . .

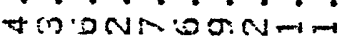
$\forall$ o monses os

mamma mand $v \rightarrow 100 m-7$

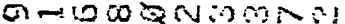

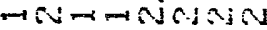

MUNMNNNDS $-m-i x-1-1 \rightarrow-1, N$ $\omega \infty \omega \infty \infty \infty 00000$ Q

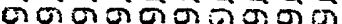
NANRNANR

ND - -1 NNNSNNON

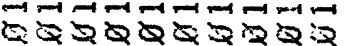


APPENDIX D

Publication in Geology by Khattri and Wyss 


\title{
Precursory variation of seismicity rate in the Assam area, India
}

\author{
K. Khattri,* M. Wyss \\ Cooperative Institute for Research in Environmental Sciences \\ University of Colorado/NOAA, Boulder, Colorado 80309
}

\begin{abstract}
The seismicity data from 1825 to the present for the Assam (northeastern India) region show that seismicity rates there deviate from normal before and after major earthquakes. Along this $1,000-\mathrm{km}$-long section of a plate boundary, all shocks with magnitude $M>6.6$ were preceded and sometimes followed by periods of significant seismic quiescence. No major earthquakes occurred without an associated seismic quiescence, and no such quiescence occurred at times other than before or after a major event. The most remarkable periods of quiescence lasted about 28 and 30 yr before the two great $(M=8.7)$ Assam earthquakes of 1897 and 1950 . Other periods of anomalously low seismicity preceded main shocks of magnitudes 6.7 (in 1950 and 1975), 7.8 (in 1869), and 7.7 (in 1947), with durations of $6,8,23$, and $17 \mathrm{yr}$, respectively. These durations fit (with approximately the scatter of the original data) a published relation between precursor time and magnitude.

Since these changes of seismicity rate were observed at the edges of and within the Assam gap, defined by the 1897 and 1950 great earthquakes, it is likely that a future major or great earthquake in this gap will be preceded by seismic quiescence. Whether a preparatory phase for an earthquake has begun in the Assam gap cannot be stated for certain because of the changing earthquake-detection capability in the area and because of poor location accuracy.
\end{abstract}

\section{INTRODUCTION}

The seismicity pattern in space and time was probably the most important factor that led to the successful prediction of the Haicheng earthquake (Anonymous, 1976). In that case, the migration of medium-magnitude events toward Haicheng alerted local researchers to monitor the area, and the immediate foreshocks indicated that an earthquake warning should be issued.

The idea of seismic gaps has been successfully used to identify areas of increased earthquake risk and to estimate approximate source dimensions of the expected ruptures (Fedotov, 1967; Mogi, 1969; Sykes, 1971; Kelleher and others, 1973). Seismic gaps are sections of plate boundaries where no large earthquakes have occurred for a duration comparable with the recurrence time. Even after numerous small- and medium-magnitude earthquakes occur in a gap, it must still be considered a place of high stress concentration, because small earthquakes, unless associated with additional seismic creep, do not relieve a significant amount of tectonic strain compared to large ones [a fact that can be checked by summing the seismic moments of the square

*Present address: Geology and Geophysics Department, Roorkee University, Roorkee, U.P. 247672, India. root of energy released by earthquakes in a given area (Benioft, 1951; Brune, 1968)]. For this reason, seismic gaps can be defined successfully using the large earthquakes only. However, it may be that the smaller "background" earthquakes hold the key to the third element necessary for successful prediction: the time of occurrence. This may be determinable once the relation of the main shocks and their precursory seismicity fluctuations (Nersesov and others, 1973; Brady, 1974, 1976, 1977; Ishida and Kanamori, 1977; Evison, 1977; McNally, 1977) is understood. In addition to these long-term seismicity patterns, Jones and Molnar (1976) found that about $40 \%$ of large earthquakes have foreshocks that occur hours and days before the main event.

The area of interest in this paper is the Assam region (inset in Figs. 1, 4). In this northeasternmost part of India, two great earthquakes of magnitude 8.7 have occurred in the past $80 \mathrm{yr}$, leaving a seismic gap between them, which we will call the Assam gap. In this area the Himalaya are interpreted to form a clearly defined arcuate zone of plate consumption, along which the Indian and Asian plates collide at a rate of about $5 \mathrm{~cm} / \mathrm{yr}$. Since the Assam gap is one of very few clearly defined thrust gaps on land, it is one of the few locations where the Earth's behavior precursory to a great thrust earthquake may be monitored in detail. As a first step we have examined the seismicity record of the Assam region for the past $150 \mathrm{yr}$. 


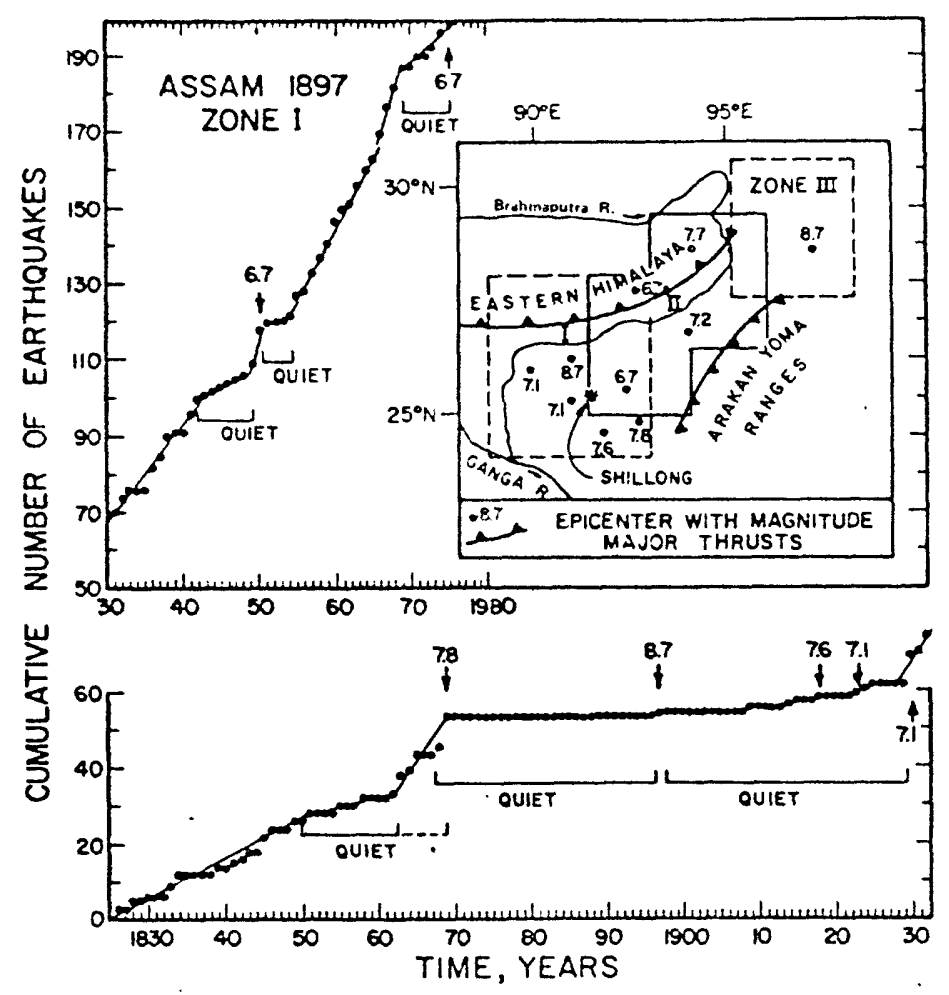

Figure 1. Cumulative number of earthquakes in zone I of the Assam area covering the extent of the great 1897 earthquake $(M=8.7)$. The inset shows a map of eastern India with the zones of study. The resolution (lowest magnitudes, $M_{\mathrm{min}}$, at which all occurring earthquakes are reported) is estimated from the frequency magnitude relation of the data as follows: for 1816 to $1909, M_{\min }=7.0$; for 1910 to $1930, M_{\min }$ $=6.8$; for 1931 to $1950, M I_{\min }=6.2$; for 1951 to $1962, M_{\mathrm{min}}=5.8$; and for 1963 to $1976, M_{\min }=4.8$. This inhomogeneity will not pose a problem for our study, because we are searching for anomalous decreases of seismic activity, which is opposite to the trend of generally increasing reports with time.

The tectonic regime of the Assam region can be summarized as a south-directed overthrusting from the north and a northwest-directed overthrusting from the southeast in the Shillong Plateau area that result from the collison of India with Asia (see Tapponnier and Molnar, 1977). Farther to the northeast the direction of compression from the north turns around to align in a northwest-southeast direction. The seismicity appears to be somewhat diffused, which may in part be due to imprecise location of earthquake epicenters. Even so, the epicenters seem to outline some of the major tectonic lineaments. The earthquakes are generally confined to the upper $80 \mathrm{~km}$ except in the Arakan Yoma ranges to the southeast, where hypocenters as deep as $250 \mathrm{~km}$ have been reported. The northeasternmost part of the area, the locale of the 1950 great earthquake, is the site of the eastern syntaxis in the Himalayan chain.

\section{DATA}

In order to investigate seismicity, the $1,000-\mathrm{km}$ segment of the plate boundary in Assam was divided into three somewhat overlapping zones: zone I enclosing roughly the mezoseismal area of the great earthquake of 1897 , zone II defining the gap between the two great earthquakes of 1897 and 1950, and zone III covering the seismic activity associated with the great 1950 earthquake (inset in Fig. 1). The overlap was considered desirable to achieve some continuity in the data analysis.

Aftershocks must be excluded in seismicity studies like the

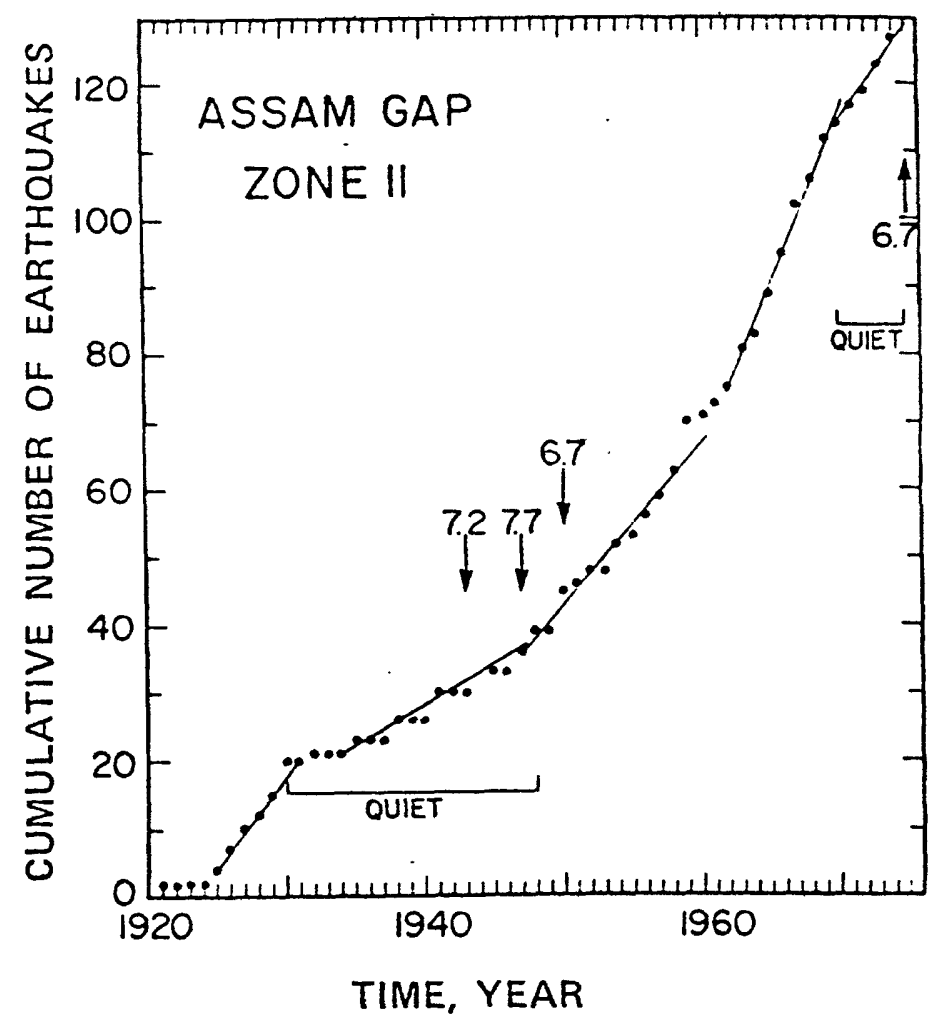

Figure 2. Cumulative number of earthquakes located in zone II, the area of the Assam gap, a region located between two great historic earthquakes but within which no great quake has been recorded.

present one because we consider the main shock-aftershock sequence as one event only, and because an overestimate of the background activity would result if aftershocks are not excluded. However, in our case, aftershocks in the data set do not pose a problem, because we consider a time scale with 1 yr as the smallest unit and $5 \mathrm{yr}$ as the minimum for defining local seismicity trends. Furthermore, for main shocks before 1925, where the seismicity is obtained from felt reports, the aftershocks were not counted or reported. For these reasons it is sufficient to make only one fairly crude adjustment of the data: we assumed that all earthquakes reported in zone 111 from 1950 through 1951 were aftershocks of the great 1950 main event, and we omitted these quakes from Figure 3.

The source of seismicity data back to 1900 is the data file of NOAA's National Geophysical and Solar Terrestria! Data Center. The seismicity data for the period before 1900 are from the catalogue compiled by Roorkee University (unpublished), which is the most complete set of historical seismicity data for this area.

The cumulative number of the earthquakes reported are plotted for the three regions in Figures 1, 2, and 3, respectively. Approximate seismicity trends that seemed to be constant over several years are indicated by fine lines, which were fitted by eye through the data. The occurrence time of all the reported earthquakes with $M \geq 6.7$ is indicated by arrows for the time after 1940. For the earlier period, only outstandingly large quakes (usually $>7.5$ ) were marked.

The most important result becomes immediately obvious. Each large earthquake in any of the three regions was preceded by a period of low seismicity. These periods are very pronounced, with their lengths apparently a function of magnitude. A period of "significant quiescence" will be defined as one in 


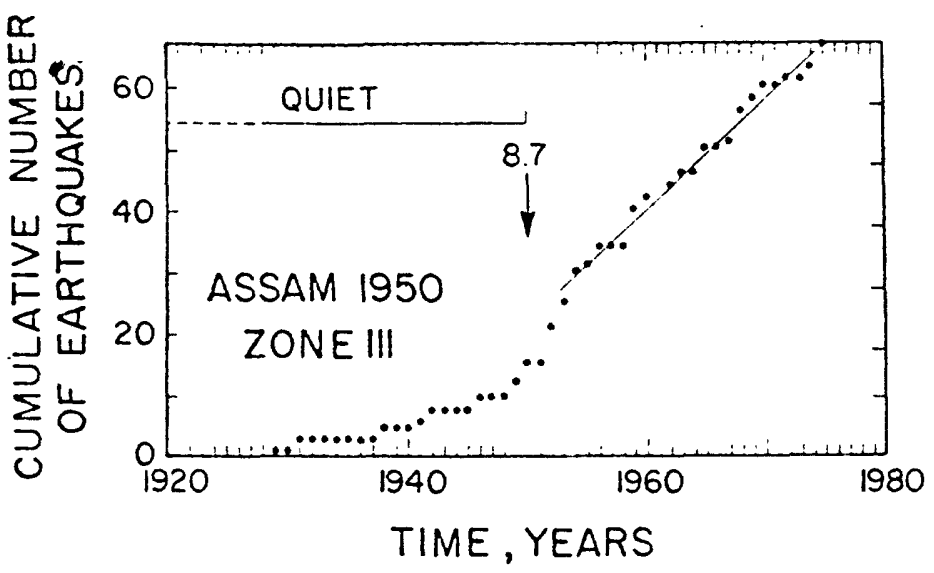

Figure 3. Cumulative number of earthquakes located in zone III, the area of the great 1950 Assam earthquake $(M=8.7)$.

Figure 4. Map of epicenters in the Assam gap from 1950 to 1975. Aftershock areas of great earthquakes are shaded. The area of low seismicity outlined by dashes may be the most likely place for one or two future large earthquakes.

which during more than $3 \mathrm{yr}$ the seismicity rate was less than 0.6 the rate defined by the 5 to $10 \mathrm{yr}$ preceding it. The periods that thus qualify as "quiet" are labeled in Figures 2 and 3.

Zone I was chosen to include the great Assam earthquake of 1897. It is remarkable that not a single event was reported in that area during the $28 \mathrm{yr}$ before this great earthquake. For comparison, during the $28 \mathrm{yr}$ preceding this period of quiescence, 38 earthquakes were reported. Just as outstanding is the remarkable period of quiescence from 1899 to 1930 that was terminated by the sequence of a magnitude 7.6 and two 7.1 earthquakes. After these events, the background level of seismicity sharply increased.

The more recent seismicity data for the area (upper curve in Fig. 1) define very clearly an 8-yr and a 6-yr period of relative quiescence before the magnitude 6.7 events in 1950 and 1975, respectively, and a 4-yr period of quiescence immediately following the 1950 earthquake. These fluctuations could not have been due to a change in detection capability, because starting in 1963 the World Wide Seismograph Network provided uniform coverage of the area, and because world-wide seismicity coverage during the 1940s was better than in the 1930s.

Anomalously low seismicity before the 1869 magnitude 7.8 earthquake is less well defined. It appears that a period of relatively low seismicity may have started in 1848 , with a small peak of activity in 1863 to 1865 . Because of the low resolution during this early historic period and because of our ignorance of magnitudes involved, we only consider a period significantly quiescent if the decreased rate lasted for $8 \mathrm{yr}$, double the requirement of more modern data. We suggest that a quiet period of about 23 yr may have preceded this earthquake.

Zone II defines the Assam gap. Zones II and III are more remote than zone I; therefore, we have to rely on instrumentally recorded seismicity for which catalogues start around 1920. Two earthquakes with magnitudes 7.2 and 7.7 occurred in zone II in 1943 and 1947 , respectively. They were preceded by a period of quiescence that started in 1930 (Fig. 2), 13 to 17 yr before these shocks. The larger of these two quakes was located north of the Himalaya thrust, that is, outside of the Assam area. An earth-

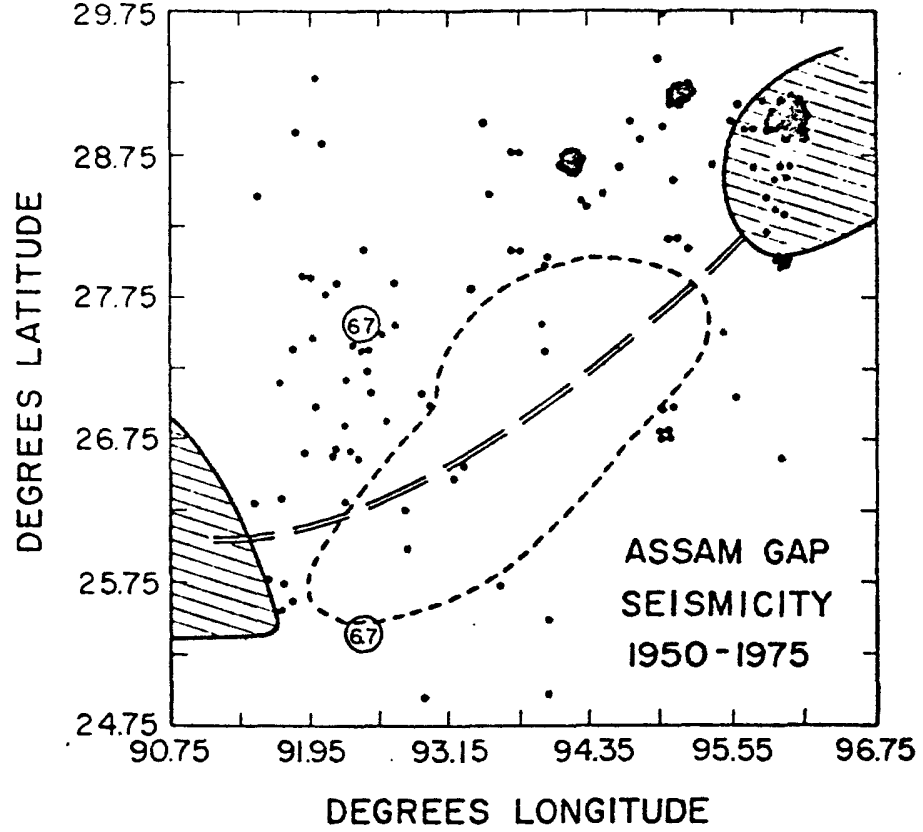

quake that occurred in 1975 in the common region of zones I and II may also have been associated with a small decrease in activity before it. The period of quiescence before 1925 is interpreted as being due to the large quakes in the neighboring zone I during the period 1918 to 1930 , because modern data show that large quakes can decrease local seismicity out to distances of 2 or 3 source dimensions (Habermann and Wyss, 1977).

Zone III includes the area of the great 1950 earthquake. The seismicity in zone III (Fig. 3) is remarkable for its low level between 1920 and 1950. Unfortunately, the seismicity level cannot be estimated before 1920 because the area is too remote. However, the rate of activity between 1920 and 1950 is lower than in either of the two other zones during the same time. We therefore conclude that this was a period of quiescence, but we give the observation a low quality rating of C (Table 1). After 1953, seismic activity was constant. As a minor point, one may note that our scheme of excluding aftershocks did not remove all such events. Two to four years after the main shock the activity still was higher than the background has been ever since.

\section{DISCUSSION}

The data shown in Figures 1, 2, and 3 demonstrate that pronounced reduction in seismicity rate preceded the larger

TABLE 1. DURATIONS OF SEISMIC QUIESCERTE BEFORE ASSAM EARTHQUAAKS

\begin{tabular}{lcccc}
\hline Area & \multicolumn{2}{c}{ Earthquale } & $\begin{array}{c}\text { Duration of quiescence } \\
(\mathrm{yr})\end{array}$ & $\begin{array}{c}\text { Approx. quality } \\
\text { of otservation }\end{array}$ \\
\cline { 2 - 5 } Date & $\mu$ & 23 & $\mathrm{C}$ \\
& 1869 & 7.8 & 28 & $\mathrm{~B}$ \\
& 1897 & 8.7 & $19 *$ & $\mathrm{C}$ \\
& 1918 & 7.6 & $5 *$ & $\mathrm{C}$ \\
& 1922 & 7.1 & 8 & $\mathrm{~A}$ \\
& 1950 & 6.7 & 6 & $\mathrm{~A}$ \\
Zone 11 & 1975 & 6.7 & 17 & $\mathrm{~A}$ \\
Zone 111 & 1947 & 7.7 & $>30$ & $\mathrm{C}$ \\
\hline
\end{tabular}

*beguming of quiescence (decrease in seis:lic activity) assumed to be caic cf preceding iarge quake. 
earthquakes in the Assam area since 1800. Significantly, there were no periods of anomalously low seismicity rate for several years that did not either immediately precede or follow one of the large earthquakes. The facts that the above data cover more than $100 \mathrm{yr}$, that each large earthquake was preceded by quiescence, and that only one quiescent period (the one after the 1950 earthquake in zone I) was not followed by a major earthquake suggest that there is a causal connection between periods of quiescence and large carthquakes. We will therefore consider low seismicity periods in this area to be precursory anomalies indicative of major earthquakes to follow.

It is surprising that the two 6.7 magnitude quakes (Fig. 1) are preceded by a significant decrease in seismicity even though a very large area is considered. A possible reason for this observation is that most of the recent seismicity occurs along the margins of zone 1 , with little activity at the center of the source area of the magnitude 8.7 great earthquake. For the 1975 magnitude 6.7 event we found that between 1963 and 1975 about half of the events plotted in Figure 1 occurred within approximately $100 \mathrm{~km}$ from that event and that the seismicity within this radius mirrored the pattern shown in Figure 1.

Heightened precursory activity (clusters) on the time scale of days and months is not defined by the data here as it apparently is in underground mines (Brady, 1974) and before some other earthquakes (Brady, 1976; Habermann and Wyss, 1977; McNally, 1977). We cannot say for sure that such precursory clusters do not exist here, because owing to their relatively small magnitude, they may simply not have been recorded for this region. In the absence of pronounced clusters, we define the precursor time as the duration from the beginning of the seismicity decrease to the large carthquake terminating the anomalously quiescent period (Table 1). On the plot of precursor time versus earthquake magnitude these values agree approximately with the curve originally proposed by Scholz and others (1973). Some of the precursor times reported here are among the longest ones observed to date, and they are for the largest earthquakes for which precursors have been noted.

It would be of great value if future earthquakes could be anticipated on the basis of the patterns observed before past earthquakes. One difficulty in this is the fact that with time more small earthquakes are being located with improving station coverage. This resolution increase is reflected by an increase in slope (Fig. 1, 3), so we do not know what amount of activity during the quiescent periods before the $M=8.7$ earthquakes would have been resolved with today's capabilities.

The epicenters of earthquakes that occurred since 1950 in the gap are shown in Figure 4, which exhibits a rather welldefined area of quiescence. With a curve through the past great earthquakes and parallel to the Himalaya mountain ranges (double dashed in Fig. 4), we suggest a zone for the likely locations of future great earthquakes. This curve runs through the area of quiescence. One possible explanation of this observation is that the instrumental epicenter locations may be biased toward the concave side of the plate boundary, as is commonly the case in subduction zones. The areas of the great earthquakes, on the other hand, are known from the local shaking effects. If the small earthquakes are mislocated by $50 \mathrm{~km}$, it could be that the apparently quiet part of the gap is not quiet at all. Alternatively, it is possible that there really exists a quiet part of the gap. The data available do not warrant an earthquake prediction. Perhaps detailed field studies in the area of the Assam gap could help to better assess the earthquake risk there.

\section{REFERENCES CITED}

Anonymous, 1976, A brief summary of the work of premonitory observation, prediction and precautionary measures before the Haicheng earthquake, Liaoning province, of magnitude 7.3: Scientia Geulogica Sinica, no. 2, p. 120.

Benioff, H., 1951, Global strain accumulation and release as revealed by great earthyuakes: Seismological Society of America Bulletin, v. 62, p. 331 .

Brady, B. T., 1974, Seismic precursors before rock failures in mines: Nature, v. 252, p. 5483.

1976, Theory of earthquakes IV. General implications of earthquake prediction: Pure and Applied Geophysics, v. 114, p. 1031. 1977, Anomalous seismicity prior to rock bursts: Implications for earthquake prediction: Pure and Applied Geophysics, v. 115 , p. 357.

Brune, J. N., 1968, Seismic moment, seismicity and rate of slip along major fault zones: Journal of Geophysical Research, v. 73, p. 777.

Evison, F. F., 1977, Fluctuations of seismicity before major earthquakes: Nature, v. 266, p. 710 .

Fedutov, S. A., 1967 , Long range seismic forecasting for the Kurile. Kamchatka zone: Transactions of Meeting of Far East EarthScience Division, Academy of Sciences USSR, Moscow.

Habermann, R. E., and Wyss, M., 1977, Seismicity patterns before five major earthquakes: EOS (American Geophysical Union Transactions), v. 58, p. 1194.

Ishida, M., and Kanamori, H., 1977, The spatio-temporal variation of seismicity before the 1971 San Fernando earthquake, California: Geophysical Research Letters, v. 4, p. 345.

Jones, L., and Molnar, P., 1976, Frequency of foreshocks: Nature, v. 262, p. 677.

Kelleher, J., Sylies, L., and Oliver, J., 1973, Possible criteria for predict. ing earthquake locations and their application to major plate boundaries of the Pacific and the Caribbean: Journal of Geophysical Research, v. 78, p. 2547.

McNally, K., 1977, Patterns of earthquake clustering preceding moderate earthquakes, central and southern California: EOS (American Geophysical Union Transactions), v. 58, p. 1195.

Mogi, K., 1969, Some features of recent seismic activity in and near Japan, 2, Activity before and after great eartinquakes: Tokyo University, Earthquake Research Institute Bulletin, v. 47, p. 395.

Nersesov, 1. L., and others, 1973, Possibilities of earthquake prediction, exemplified by the Garm area of the Tadzhik SSR, in Sadovsky, M. A., and others, eds., Earthquake precursors: Academy of Sciences USSR, p. 72.

Scholz, C. H., Syties, L. R., and Aggarwal, Y.P.A., 1973, Earthquake prediction: A physical basis: Science, v. 181. p. 803 .

Sykes, L. R., 1971, Aftershock zones of great earthquakes, seismicity gaps, and earthquake prediction for Alaska and the Aleutians: Journal of Geophysical Research, v. 76, p. 8021.

Tapponnier, P., and Molnar, P., 1977, Active faulting and tectonics in China: Journal of Geophysical Research, v. 82, p. 2905.

\section{ACKNOWLEDGMENTS}

Reviewed by K. McNally. Supported by U.S. Geological Survey Grant 14-08-0001-16841. E. R. Engdahl, W. Gawthrop, and S. Wier read the manuscript critically and offered valuable suggestions. We thank M. Carr for her help in preparing the manuscript.

MANUSCRIPT RECEIVED APRIL 14, 1978

MANUSCRIPT ACCEPTED AUGUST 28, 1978 